\title{
INFERÊNCIA EM CONFIABILIDADE DE SOFTWARE UTILIZANDO O MODELO DE GOEL E OKUMOTO COM CAPTURA-RECAPTURA
}

\author{
Luciano Barbosa
}

Orientador: Prof. Dr. Josemar Rodrigues

Dissertação apresentada ao Instituto de Ciências Matemáticas de São Carlos - USP, como parte dos requisitos para a obtenção do Título de Mestre em Ciências - Área: Ciências de Computação e Matemática Computacional.

USP

Setembro - 1997 
"O sacrificio de subir a montanha,

é compensado pela paisagem que

descortinamos do alto."

Dinamor

Dedica este trabalha

acs mens pais

Sebastiãa e Lucilia. 


\section{A GRA D E CIMENTOS}

A Deus por mais esta realização em minha vida.

Ao Prof. Dr. Josemar Rodrigues, pela orientação deste trabalho, pelo incentivo e pela constante demonstração de amizade e apreço.

Ao Prof. Dr. José Galvão Leite pelas valiosas sugestões na parte de processos de Poisson, inclusive na obtenção da média e variância de $\mathrm{N}_{\mathrm{p}}$, e pelos comentários dados no meu exame de qualificação.

Ao Prof. Dr. Marinho Gomes de Andrade pelas sugestões e comentários dados quando da realização do meu exame de qualificação e ao Prof. Dr. Jorge Alberto Achcar pelo apoio demonstrado durante as aulas e seminários.

Ao $\mathrm{CNPq}$ pelo apoio financeiro.

Aos professores, funcionários, colegas de pós-graduação e a todos aqueles que, de alguma forma, contribuíram para a realização deste trabalho. Em especial aos amigos, José Roberto, Karin, Daniela e Daniele pela amizade, carinho e apoio durante este período, dentro e fora do ICMSC.

Especialmente à Dona Antonieta e à minha namorada Rose pelo carinho, paciência e incentivo. 


\section{Resumo}

Nesta dissertação de mestrado, apresentamos uma abordagem clássica e Bayesiana para o modelo de Captura-Recaptura proposto por Nayak (1988) para obter informação sobre o número de falhas, $\mathrm{N}$, em um sistema de confiabilidade. A probabilidade de corrigir um erro, p, é assumida ser conhecida e desconhecida. Mostramos que o e.m.v. de $\mathrm{N}$ depende não somente das freqüências dos erros detectados, como também dos tempos entre falhas.

A 'sensibilidade' da distribuição a posteriori de $\mathrm{N}$ com respeito a $\mathrm{p}$ e a influência dos tempos entre falhas, são considerados através da distância da variação total e divergência de Kullback-Leibler. Também mostramos que a correção por recaptura é uma condição necessária para a existência da distribuição a posteriori de $\mathrm{N}$ quando utilizamos uma priori imprópria. 


\section{Abstract}

In this dissertation we describe a likelihood and Bayes approaches for the recapture debugging design proposed by Nayak (1988) to get information about the number of faults, $\mathrm{N}$, in a reliability system. It is considered that the debugging is only sucessful with known and unknown probability p. It is shown that the mle of $\mathrm{N}$ depend not only of the frequencies of detect bugs but also of the times between bugs.

The sensibility of the posterior distribution of $\mathrm{N}$ with respect to $\mathrm{p}$ and the influence of the time data are numerically considered via variational distance and Kullback-Leibler divergence. Also, it is shown that the recapture debugging is a necessary and sufficient condition for the existence of the posteriori distribution of $\mathrm{N}$ when an improper priori is imposed. 


\section{ÍNDICE}

\section{INTRODUÇÃO À CONFIABILIDADE DE SOFTWARE}

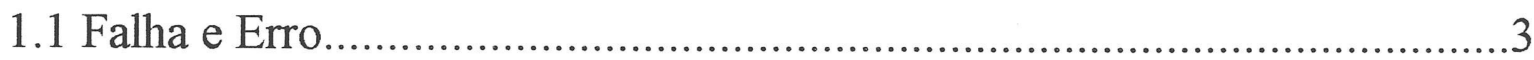

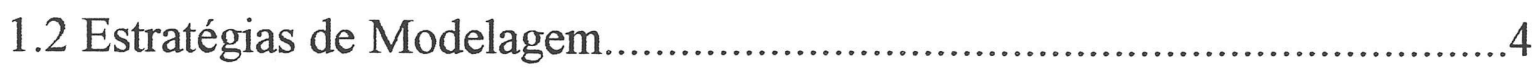

1.2.1 A Estratégia do Tipo I-1 ..................................................4

1.2.2 A Estratégia do Tipo I-2 ....................................................

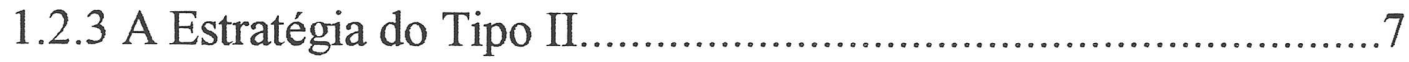

1.3 Estimador de Máxima Verossimilhança..............................................8

1.3.1 Função de Verossimilhança Profile..........................................10

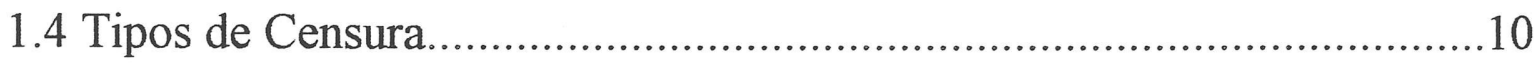

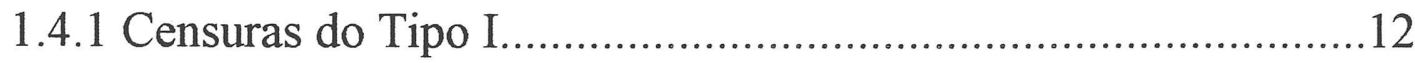

1.4.2 Censuras do Tipo II.............................................................12

1.5 Alguns Modelos de Confiabilidade de Software...................................14

1.5.1 O Modelo de Jelinski e Moranda..............................................14

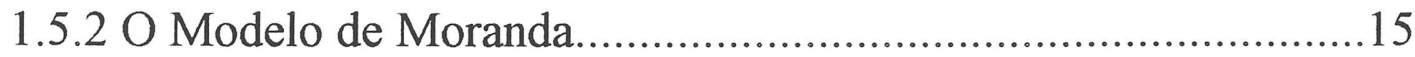

1.5.3 O Modelo de Schick e Wolverton.............................................16

1.5.4 O Modelo de Goel e Okumoto................................................17

1.5.5 O Moldeo de Captura-Recaptura de Nayak..............................18

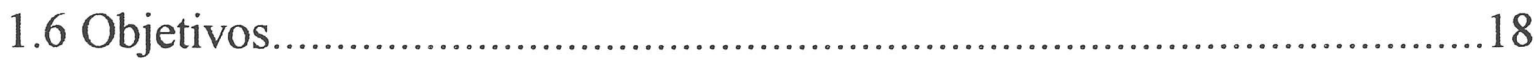




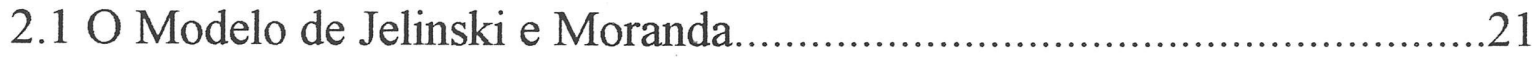

2.1.1 Função de Verossimilhança....................................................21

2.1.2 Estimadores de Máxima Verossimilhança................................23

2.1.3 Função de Verossimilhança Profile..........................................24

2.1.3.1 Inferências sobre os Parâmetros do Modelo.................25

2.2 O Modelo de Captura-Recaptura de Nayak........................................27

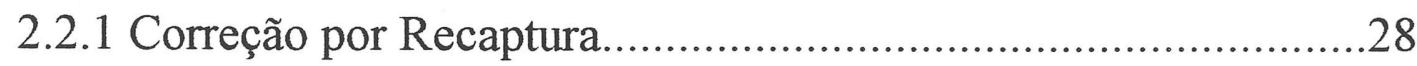

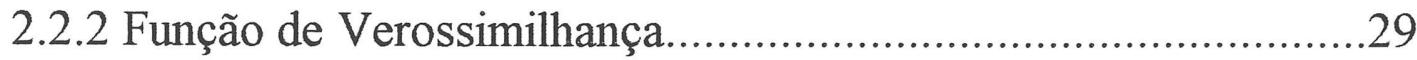

2.2.3 Estimadores de Máxima Verossimilhança................................30

2.2.4 Função de Verossimilhança Profile...........................................31

2.2.4.1 Inferências sobre os Parâmetros do Modelo..................33

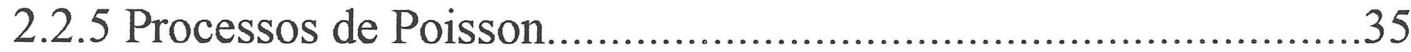

2.2.5.1 Obtendo o Modelo de Captura-Recaptura via Processos

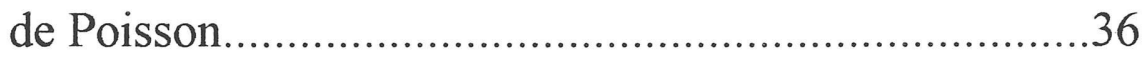

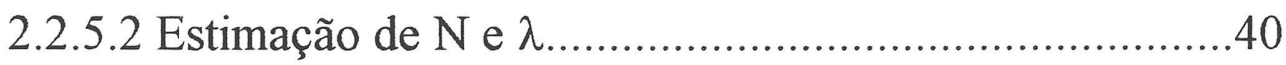

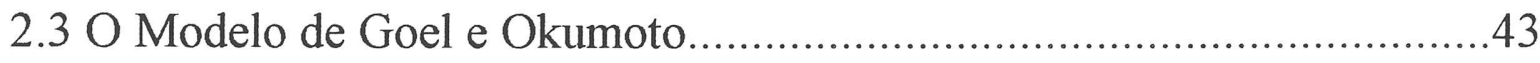

2.3.1 Função de Verossimilhança........................................................43

2.3.2 Estimadores de Máxima Verossimilhança................................44

2.3.2.1 Inferências sobre os Parâmetros do Modelo..................45

2.4 O Modelo de Goel e Okumoto com Captura-Recaptura........................47

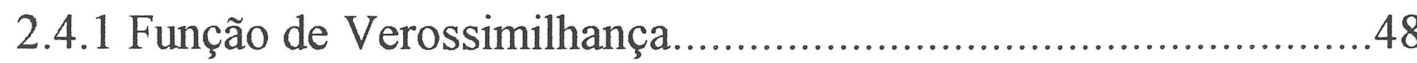

2.4.2 Estimadores de Máxima Verossimilhança................................50

2.4.3 Função de Verossimilhança Profile para N (p conhecido).........51

2.4.3.1 Estimativa da Média e Variância de $\hat{\mathrm{N}}_{\mathrm{p}}$.....................53 


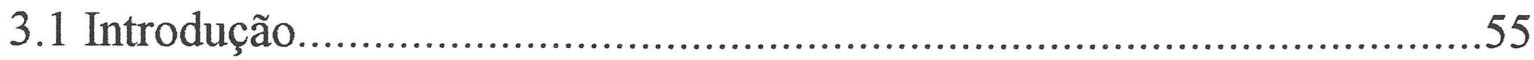

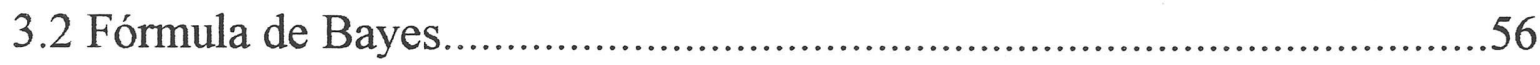

3.3 Densidades a Priori e a Posteriori para os Parâmetros...........................56

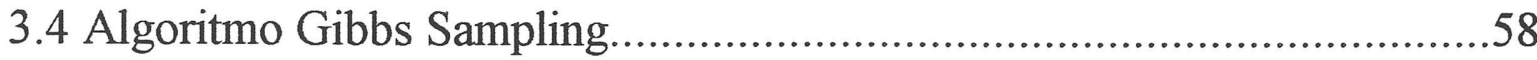

3.4.1 Verificação da Convergência..................................................60

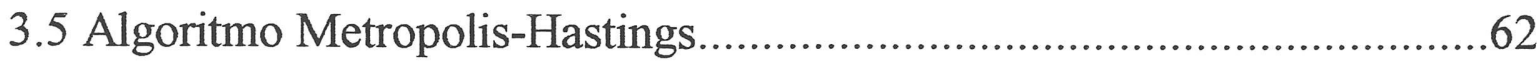

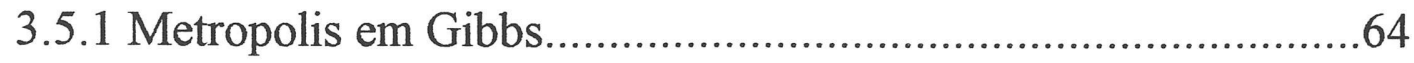

3.6 Análise Bayesiana do Modelo de Jelinski e Moranda............................65

3.7 Análise Bayesiana do Modelo de Captura-Recaptura de Nayak............68

3.8 Análise Bayesiana do Modelo de Goel e Okumoto.................................71

3.9 Análise Bayesiana do Modelo de Gole e Okumoto com Captura -

Recaptura. .74

\section{UMA ANÁLISE DA DISTRIBUIÇÃO A POSTERIORI DE N VIA MEDIDAS DE DIVERGÊNCIA}

4.1 Introdução 82

4.2 Comparando as Densidades a Posteriori Marginais de N ( $\mathrm{p}$ conhecido).83

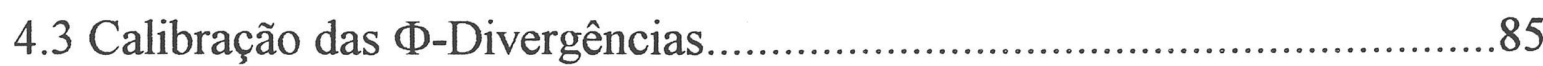

4.3.1 Calibração da Distância da Variação Total.................................86

4.3.2 Calibração da Divergência de Kullback-Leibler.........................87

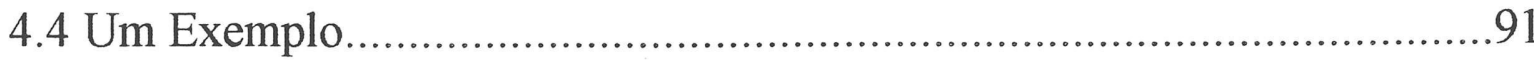


5 EXEMPLOS DE APLICAÇÃO. .94

5.1 Exemplo Considerando o Modelo de Jelinski e Moranda.......................94

5.2 Exemplo Considerando o Modelo de Captura-Recaptura de Nayak.....102

5.3 Exemplo Considerando o Modelo de Goel e Okumoto.........................108

5.4 Exemplo Considerando o Modelo de Goel e Okumoto com Captura -

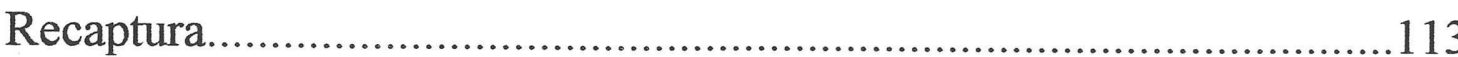

5.5 Exemplos Utilizando as Medidas de Divergência..................................121

6 CONCLUSÕES FINAIS E PERSPECTIVAS FUTURAS...........................125

Apêndice 1: O Método de Newton...................................................................127

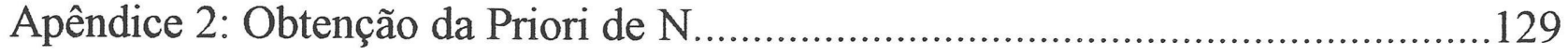

Apêndice 3: Alguns Programas Desenvolvidos......................................................130

REFERÊNCIAS BIBLIOGRÁFICAS.........................................................136 


\section{CAPÍTULO 1}

\section{INTRODUÇÃO À CONFIABILIDADE DE SOFTWARE}

Confiabilidade de software é uma área de pesquisa relativamente nova. Seu objetivo é quantificar a execução do software, o qual está sujeito a falhas devido à inevitável presença de erros de sintaxe ou lógica.

Com a crescente demanda por serviços computacionais nos últimos anos, surgiu uma série de problemas relacionados com a confiabilidade de software. Em particular, o problema de estimar o número de erros no software e a taxa de falhas tem sido estudado com muito interesse pelos estatísticos.

A competição que as empresas enfrentam torna-as severamente atentas às necessidades de produção de software. Três das mais significantes necessidades são: alto nível de qualidade, tempo de liberação e custo.

Um grande número de sistemas opera em tempo real. Nesses sistemas, os efeitos operacionais de falhas são maiores e geralmente críticos. Por exemplo, considere os efeitos de um colapso nas reservas aéreas, negócios bancários, controle automático de vôo, defesa militar, e usinas nucleares seguramente controladas por sistemas. Os resultados econômicos podem ser maiores e catastróficos. Os custos das falhas incluem não somente gastos diretos mas também a responsabilidade de risco dos produtos e danos à reputação de uma companhia. 
O ritmo acelerado de mudanças na tecnologia de computação faz que sistemas de informação se tornem economicamente obsoletos mais rapidamente. O software pode tornar-se obsoleto também pelo resultado da nova tecnologia de hardware ou nova tecnologia de software. Portanto, o tempo de liberação de um sistema temse tornado significativamente crucial. A "janela de mercado", ou período de tempo disponível para a introdução de um novo produto, antes que este seja ultrapassado em capacidade ou custo, tem diminuído.

Com a pressão do custo e do tempo fica impossível criar um software que seja "generoso" no sentido de dar simultaneamente alta qualidade (confiabilidade), rápida liberação e baixo custo, mas tais características são os objetivos desejados.

Estão presentes no software erros que podem motivar o programa a produzir resultados contraditórios às suas especificações. Essa discrepância que existe entre o que o software pode fazer versus o que o usuário (ou o programador) quer que ele faça é chamada falha do software.

Modelos probabilísticos e métodos estatísticos estão sendo estudados para avaliar a confiabilidade do software do computador. O objetivo desses modelos é quantificar a confiança do software de uma maneira exata quanto possível, fazendo suposições realistas sobre o processo básico de falhas.

Estatísticos usam esses modelos para medir a confiabilidade, analisar dados de falhas, fazer inferências sobre o procedimento futuro do software e tomar decisões durante o processo de teste do software, como por exemplo, quantos erros devem 
ocorrer no máximo para que ele possa ser liberado para o consumo; ou qual deve ser o seu tempo de teste, etc.

\subsection{Falha e Erro}

Aqui nós devemos fazer uma distinção entre erros e falhas no software. Um erro existiria indefinidamente em um pedaço do software sem ser a causa da falha.

Suponha-se que estejamos executando um programa (software) e de repente ele pára de funcionar ou não dá a resposta que esperamos. Isso chama-se falha do software. Note que uma falha não é a mesma coisa que um erro. O erro é um defeito no programa, que quando executado sobre certas condições, causa uma ou mais falhas. Um erro pode ou não originar falhas.

O custo da reparação de um erro é geralmente pequeno em relação ao impacto do custo operacional da falha. O impacto da falha nos custos é particularmente aplicável a algum sistema que está operando no mundo dos negócios. O impacto na vida humana ocorre, por exemplo, nas usinas nucleares, nos sistemas de controle de tráfego aéreo. E o impacto da falha nos sistemas pode se dar nos sistemas de telefonia.

Portanto, o estudo da confiabilidade do software é extremamente importante no sentido de diminuir ou eliminar esses e muitos outros impactos causados pelas falhas dele. 


\subsection{Estratégias de Modelagem}

Existem duas estratégias de modelagem amplamente utilizadas em confiabilidade do software. Elas não cobrem todos os modelos propostos, mas a maioria deles. A estratégia do tipo I modela os tempos entre falhas sucessivas do software, e pode ser dividida em dois subtipos distintos; e a estratégia do tipo II modela o número de falhas do software por um determinado tempo.

\subsubsection{A Estratégia do Tipo I-1}

Quando o modelo é definido através das taxas de falhas para os tempos entre falhas sucessivas, temos a estratégia de modelagem do tipo I-1.

Seja $\mathrm{T}$ a variável aleatória que denota o tempo da primeira falha. A probabilidade de não falhar até o tempo t é dada pela função de confiabilidade

$$
R(t)=P(T \geq t)=\int_{t}^{\infty} f(x) d x
$$

Note que $\mathrm{R}(\mathrm{t})$ é uma função monótona contínua decrescente com $\mathrm{R}(0)=1$ e $R(\infty)=\lim _{t \rightarrow \infty} R(t)=0$.

Se $\mathrm{P}(\mathrm{T} \geq \mathrm{t})$ é diferenciável em relação a t, o negativo da derivada é a função de densidade de probabilidade de $T$, denotada por $f(t)$, isto é, 


$$
f(t)=-\frac{d R(t)}{d t} .
$$

A razão de $f(t)$ por $\mathrm{P}(\mathrm{T} \geq \mathrm{t})$ é definida como a função de risco ou taxa de falha, isto é,

$$
h(t)=\lim _{\Delta t \rightarrow 0} \frac{P(t \leq T<t+\Delta t / T \geq t)}{\Delta t}=\frac{f(t)}{R(t)} .
$$

A função de risco especifica a taxa instantânea de falha em $\mathrm{T}=\mathrm{t}$, dado que o software não falhou até o instante $t$. Em particular, $h(t) \Delta t$ é a probabilidade aproximada de falhar no intervalo $[t, t+\Delta t)$, já que não falhou até o tempo $t$.

Também podemos definir a função de risco como

$$
h(t)=-\frac{d \ln [R(t)]}{d t}
$$

A função de risco acumulado é expressa por

$$
H(t)=\int_{0}^{t} h(x) d x
$$

Segundo as definições (1.4) e (1.5), temos a relação

$$
R(t)=\exp \left\{-\int_{0}^{t} h(x) d x\right\}
$$


Assim, a função densidade de probabilidade de $T$ (ver Kalbfleisch e Prentice, 1980), pode ser representada por

$$
f(t)=h(t) \exp \left\{-\int_{0}^{t} h(x) d x\right\}
$$

EXEMPLO 1: Considere uma distribuição exponencial com parâmetro $\theta$, cuja função densidade é dada por

$$
f(t)=\frac{1}{\theta} \mathrm{e}^{-t / \theta}, \mathrm{t}>0, \theta>0 .
$$

A função distribuição acumulada é dada por

$$
F(t)=1-e^{-t / \theta}
$$

onde $\theta$ é o tempo médio de falha.

A média e a variância de $T$ são $E(T)=\theta$ e $\operatorname{Var}(T)=\theta^{2}$.

As funções de confiabilidade e de risco são dadas, respectivamente, por:

$$
R(t)=1-F(t)=e^{-t / \theta} \text { e } \quad h(t)=\frac{1}{\theta}
$$




\subsubsection{A Estratégia do Tipo I-2}

Uma outra alternativa para modelar o tempo entre falhas é definir uma relação estocástica entre os sucessivos tempos de falhas. Esta estratégia, que modela diretamente os tempos entre falhas sem preocupar-se com as taxas de falhas, é chamada estratégia do tipo I-2.

Por exemplo, sejam $\mathrm{W}_{1}, \mathrm{~W}_{2}, \ldots, \mathrm{W}_{\mathrm{i}}, \ldots$ variáveis aleatórias denotando o tempo entre sucessivas falhas do software. Como num simples caso, poderíamos declarar que $\mathrm{W}_{\mathrm{i}+1}=\rho \mathrm{W}_{\mathrm{i}}+\varepsilon_{\mathrm{i}}$, onde $\rho \geq 0$ é uma constante e $\varepsilon_{\mathrm{i}}$ é um termo de distúrbio (alguma típica variável aleatória com média 0 ). Então $\rho<1$ indicaria decréscimo dos tempos entre falhas, ou seja, a confiabilidade do software piorou; $\rho=1$ indicaria nenhuma mudança na confiabilidade do software, enquanto que $\rho>1$ indicaria aumento nos tempos entre falhas, ou seja, a confiabilidade do software melhorou.

\subsubsection{A Estratégia do Tipo II}

A estratégia do tipo II modela o número de falhas do software para um dado tempo. Seja $M(t)$ o número de falhas do software que são observadas durante o tempo $[0, t)$. $M(t)$ é modelado por um processo de Poisson com função de valor médio $\mu(\mathrm{t})$, onde $\mu(\mathrm{t})$ é não-decrescente. 
Os diferentes modelos deste tipo especificam uma diferente função $\mu(t)$. O processo de Poisson é escolhido por ser um processo pontual simples e por ter muitas propriedades úteis que podem ser exploradas.

O número de falhas do software, $\mathrm{M}(\mathrm{t})$, pode também ser especificado por sua função intensidade $\lambda(t)$, a qual é a derivada de $\mu(t)$ em relação a t. Se $\lambda(t)$ é uma constante $(\mu(t)$ é linear), então $M(t)$ é chamado de processo de Poisson homogêneo; caso contrário, é chamado de processo de Poisson não-homogêneo.

\subsection{Estimador de Máxima Verossimilhança}

Seja $\mathrm{X}$ uma variável aleatória com função densidade de probabilidade dada por $\mathrm{f}(. ; \theta), \theta \in \Theta$, onde $\mathrm{f}(. ; \theta)$ é conhecida, menos o parâmetro $\theta$. Queremos então estimar $\theta$ ou $g(\theta)$, onde $g($.$) é uma função conhecida.$

Para isso, selecionamos uma amostra aleatória $X_{1}, X_{2}, \ldots, X_{n}$ de $X$. A densidade conjunta da amostra é dada por

$$
f\left(x_{1}, \ldots, x_{n} ; \theta\right)=f\left(x_{1}, \theta\right) \ldots f\left(x_{n}, \theta\right)=\prod_{i=1}^{n} f\left(x_{i} ; \theta\right)
$$

A partir do momento em que observamos os dados, toda a informação sobre os parâmetros está contida na função de verossimilhança. A função de verossimilhança 
é a função densidade conjunta, mas pensada em termos de $\theta$, considerando-se $x_{1}, \ldots, x_{n}$ como fixados, isto é,

$$
L(\theta)=f\left(x_{1}, \ldots, x_{n} ; \theta\right)=\prod_{i=1}^{n} f\left(x_{i} ; \theta\right)
$$

Considerando-se $1(\theta)=\log [\mathrm{L}(\theta)]$, o estimador de máxima verossimilhança é o valor de $\theta, \hat{\theta}$, que maximiza $1(\theta)$.

Para obtermos $\hat{\theta}$, derivamos $1(\theta)$ em relação a $\theta$ e igualamos a zero, isto é,

$$
\frac{d[l(\theta)]}{d \theta}=0
$$

Quando temos dois parâmetros, por exemplo $\alpha$ e $\beta$, o estimador de máxima verossimilhança (e.m.v.) de $\alpha$ e $\beta$ é o par $(\hat{\alpha}, \hat{\beta})$ que maximiza $L(\alpha, \beta)$.

Análogo ao caso unidimensional, $(\hat{\alpha}, \hat{\beta})$ é um e.m.v. de $(\alpha, \beta)$ se

$$
\mathrm{S}(\hat{\alpha, \beta})=\left(\begin{array}{l}
\mathrm{S}_{1} \\
\mathrm{~S}_{2}
\end{array}\right)=\left(\begin{array}{l}
\frac{\partial \mathrm{l}(\alpha, \beta)}{\partial \alpha} \\
\frac{\partial \mathrm{l}(\alpha, \beta)}{\partial \beta}
\end{array}\right)=0
$$




\subsubsection{Função de Verossimilhança Profile}

Em muitas situações práticas, embora o modelo dependa de dois parâmetros, somente um deles, por exemplo, $\alpha$, é de interesse ( $\beta$ é o parâmetro de "nuisance"). Neste caso, usamos a função de verossimilhança profile.

A função de verossimilhança profile, $\mathrm{L}_{\mathrm{p}}(\alpha)$, é definida como

$$
\mathrm{L}_{\mathrm{p}}(\alpha)=\mathrm{L}(\alpha, \hat{\beta}(\alpha))
$$

onde $\hat{\beta}(\alpha)$ é o e.m.v. de $\beta$ para $\alpha$ fixado (ver Kalbfleisch, 1985).

\subsection{Tipos de Censura}

Existem dois tipos de conjunto de dados:

1) dados completos: tempos de falhas exatos de cada unidade amostral;

2) dados censurados: algumas unidades não falham no teste; somente é conhecida parte de seu tempo de falha (censuras à direita).

Para analisar os dados de tempos de falhas censurados, temos que levar em consideração os tipos de censuras que podem ocorrer. 
Para discutir a censura, devemos considerar a maneira de como os dados foram obtidos. O problema básico é determinar a distribuição amostral e a correspondente função de verossimilhança para um dado processo, e então determinar as propriedades dos métodos estatísticos.

EXEMPLO 2: Considere-se uma amostra aleatória de tamanho n, com tempos de falhas $T_{1}, T_{2}, \ldots, T_{n}$, exponencialmente distribuídos, com densidade (1.8). Quando temos dados completos, a função de verossimilhança da densidade exponencial em (1.8) é dada por

$$
L(\theta)=\prod_{i=1}^{n} f\left(t_{i} ; \theta\right)=\frac{1}{\theta^{n}} \exp \left(-\sum_{i=1}^{n} t_{i} / \theta\right)
$$

O logaritmo da função de verossimilhança para $\theta$ é dado por

$$
1(\theta)=\ln [L(\theta)]=-\mathrm{n} \ln (\theta)-(\mathrm{T} / \theta), \quad \text { onde } \mathrm{T}=\sum_{\mathrm{i}=1}^{\mathrm{n}} \mathrm{t}_{\mathrm{i}}
$$

Para obtermos $\hat{\theta}$, derivamos $1(\theta)$ em relação a $\theta$ e igualamos a zero. Feito isso, encontramos o estimador de máxima verossimilhança para $\theta$, que é dado por $\hat{\theta}=\mathrm{T} / \mathrm{n}$. 


\subsubsection{Censuras do Tipo I}

Existem experimentos onde o tempo de duração de um componente somente será conhecido se ele for menor do que algum valor predeterminado. Em tais situações, os dados têm o esquema de censura do tipo I.

Por exemplo, em um experimento de teste de falha de $\mathrm{n}$ componentes, toma-se a decisão de terminar o teste após um tempo $\mathrm{L}$ decorrido ( $\mathrm{L}$ fixo). Os tempos de falhas serão então conhecidos somente para aqueles componentes que falharam até o tempo L. Os componentes que continuarem em funcionamento, serão considerados censurados.

\subsubsection{Censuras do Tipo II}

Uma amostra censurada do tipo II é aquela onde somente as $r$ primeiras falhas numa amostra aleatória de tamanho $\mathrm{n}$ são observadas $(1 \leq \mathrm{r} \leq \mathrm{n}, \mathrm{r}$ fixo).

Experimentos envolvendo censuras do tipo II são geralmente usados em testes de tempos de vida. Por exemplo, um total de $\mathrm{n}$ itens é colocado em teste, mas ao invés de continuar até todos os $\mathrm{n}$ itens terem falhado, o teste termina na r-ésima falha. Esses testes podem economizar tempo e dinheiro, visto que levaria muito tempo para todos os $\mathrm{n}$ itens falharem. 
EXEMPLO 3: Considere uma amostra aleatória de tamanho $\mathrm{n}$, com tempos de falhas $T_{1}, T_{2}, \ldots, T_{n}$, exponencialmente distribuídos, com densidade (1.8). Assumindo censuras do tipo II, onde somente os $\mathrm{r}$ primeiros tempos de falhas são observados, ou seja, $\mathrm{t}_{(1)} \leq \mathrm{t}_{(2)} \leq \ldots \leq \mathrm{t}_{(\mathrm{r})}$, a função de verossimilhança para $\theta$ é dada por (ver Lawless, 1982)

$$
\begin{aligned}
& \mathrm{L}(\theta)=\left[\prod_{\mathrm{i}=1}^{\mathrm{r}} \mathrm{f}\left(\mathrm{t}_{\mathrm{i}}\right)\right]\left[\mathrm{R}\left(\mathrm{t}_{(\mathrm{r})}\right)\right]^{\mathrm{n}-\mathrm{r}} \\
& =\frac{1}{\theta^{\mathrm{r}}} \exp \left\{-\sum_{\mathrm{i}=1}^{\mathrm{r}} \mathrm{t}_{(\mathrm{i})} / \theta\right\} \exp \left\{-\frac{(\mathrm{n}-\mathrm{r}) \mathrm{t}_{(\mathrm{r})}}{\theta}\right\} \\
& =\frac{1}{\theta^{\mathrm{r}}} \exp (-\mathrm{T} / \theta),
\end{aligned}
$$

onde $\mathrm{T}=\sum_{\mathrm{i}=1}^{\mathrm{r}} \mathrm{t}_{(\mathrm{i})}+(\mathrm{n}-\mathrm{r}) \mathrm{t}_{(\mathrm{r})}$.

O estimador de máxima verossimilhança é dado por $\hat{\theta}=\mathrm{T} / \mathrm{r}, \mathrm{r}>0$.

Podemos observar que as amostras completas são consideradas um caso particular de censuras do tipo II, pois se fizermos $r=n$, teremos as mesmas expressões do exemplo 2. 


\subsection{Alguns Modelos de Confiabilidade de Software}

Existem muitos modelos de confiabilidade de software na literatura (ver por exemplo, Singpurwalla e Wilson, 1994; Goel, 1985). A seguir, veremos alguns dos mais populares e que se enquadram nas estratégias vistas anteriormente. Um problema para todos os modelos é a falta de dados para testar sua validade; por essa razão, é importante que as suposições feitas originalmente neles sejam pensadas cuidadosamente.

\subsubsection{O Modelo de Jelinski e Moranda}

Um modelo bastante conhecido e que tem servido de base para muitos outros é o desenvolvido por Jelinski e Moranda (1972), conhecido como o modelo JM.

Este modelo, de estratégia do tipo I-1, modela os tempos entre falhas do software considerando-se suas taxas de falhas. A taxa de falha do software, ou a função de risco, é tida como uma constante proporcional ao número de erros restantes no programa. A taxa de falha durante $W_{i}$ (o tempo entre a (i-1)-ésima e a i-ésima falha) é dada por

$$
h\left(\mathrm{w}_{\mathrm{i}}\right)=\lambda[\mathrm{N}-(\mathrm{i}-1)]
$$

onde $i=1,2,3, \ldots$ para alguma constante $\lambda$. 
Dessa maneira, Jelinski e Moranda consideram que cada erro contribui com uma mesma quantidade $\lambda$, para a taxa de falha, como mostra a figura (1.1).

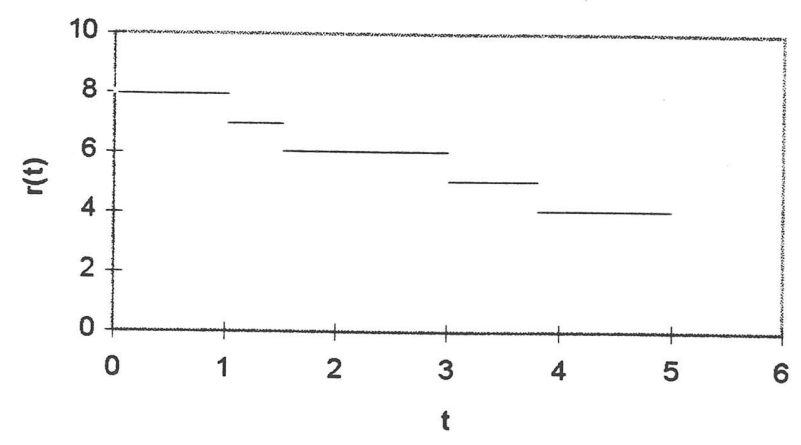

Figura 1.1 - Taxa de falha para o modelo JM.

Observando a figura (1.1), notamos que a taxa de falhas está decrescendo a cada intervalo de tempo entre sucessivos erros, e esse decréscimo é de uma quantidade $\lambda$. Este modelo será mais detalhado no capítulo 2 .

\subsubsection{O Modelo de Moranda}

Algumas modificações do modelo de Jelinski e Moranda são apresentadas na literatura. Moranda (1975) supôs que a correção dos erros que causam as primeiras falhas no software reduz mais a taxa de falha do que a correção dos que ocorrem mais tarde, porque os primeiros erros que são provavelmente os mais importantes. 
Então, Moranda assume que a taxa de falha permanece constante para cada $\mathrm{W}_{\mathrm{i}}$, mas que ela decresce geometricamente em $\mathrm{i}$ após cada falha, isto é, para constantes D e k,

$$
h\left(\mathrm{w}_{\mathrm{i}}\right)=\mathrm{Dk}^{\mathrm{i}-1},
$$

onde $\mathrm{D}>0$ e $0<\mathrm{k}<1$.

Este é o modelo eutrofia de Moranda. Comparado ao modelo JM, onde a queda na taxa de falha após cada falha era sempre $\lambda$, aqui a queda na taxa de falha após a i-ésima falha é $\mathrm{D}(1-\mathrm{k}) \mathrm{k}^{\mathrm{i}-1}$.

\subsubsection{O Modelo de Schick e Wolverton}

Esse modelo é baseado nas mesmas suposições do modelo de Jelinski e Moranda, exceto na função de risco, que é proporcional ao número de erros restantes no software e ao tempo decorrido desde a última falha. Assim, Schick e Wolverton (1978) assumem que a taxa de falha para esse modelo de estratégia do tipo I-1 é dada por

$$
h\left(\mathrm{w}_{\mathrm{i}}\right)=\lambda(\mathrm{N}-\mathrm{i}+1) \mathrm{w}_{\mathrm{i}} .
$$




\subsubsection{O Modelo de Goel e Okumoto}

Os modelos de Jelinski e Moranda (1972), Moranda (1975) e Schick e Wolverton (1978) assumem que os erros são removidos com certeza quando detectados. Porém, na prática, isso nem sempre ocorre. Pensando neste problema, Goel e Okumoto (1978) generalizaram o modelo de Jelinski e Moranda incluindo a possibilidade de que um erro seja eliminado com probabilidade $p, 0 \leq p \leq 1$. Isto é, com probabilidade (1-p) um novo erro é gerado com a mesma taxa de falha do erro anterior.

Isto significa que após, $i$ erros terem sido encontrados no software, nós esperamos que $i \mathrm{x} p$ erros sejam corrigidos, ao invés de $i$.

Este modelo é conhecido como modelo de correção imperfeita e sua taxa de falha é dada por

$$
h\left(\mathrm{w}_{\mathrm{i}}\right)=\lambda[\mathrm{N}-\mathrm{p}(\mathrm{i}-1)],
$$

onde $\mathrm{N}$ é o número de erros do software, $\mathrm{p}$ é a probabilidade de corrigir um erro e $\lambda$ é a taxa de falha por erro. Quando $\mathrm{p}=1$, temos o modelo de Jelinski e Moranda.

Neste modelo, o número de erros no software no tempo $t, X(t)$, é tratado como um processo de Markov cujas probabilidades de transição são governadas pela probabilidade da correção imperfeita. Assume-se que os tempos entre as transições de $\mathrm{X}(\mathrm{t})$ são exponenciais com taxas dependendo do erro do software. 


\subsubsection{O Modelo de Captura-Recaptura de Nayak}

Nayak (1988), supondo que $\mathrm{p}=1$ no modelo de Goel e Okumoto (1978), ou seja, que, quando o software falha, o erro causador da falha é detectado e removido sem a inserção de outros erros, adaptou um contador $M_{i}$ para o i-ésimo erro corrigido, onde $\mathrm{M}_{\mathrm{i}}$ é o número de vezes que a área $\mathrm{A}_{\mathrm{i}}$ é acessada.

Este modelo, que será visto mais detalhadamente no capítulo 2, é conhecido como modelo de captura-recaptura dos erros corrigidos, onde o contador $\mathrm{M}_{\mathrm{i}}$ é utilizado para obter informação adicional sobre $\mathrm{N}$ e $\lambda$. Este planejamento chamado correção por recaptura é proposto por Nayak (1988) para obter informação extra, para estimar o número original de falhas, $\mathrm{N}$, em um sistema de confiabilidade. $\mathrm{O}$ sistema é observado por um período fixo de tempo $\tau$ e assume-se que as falhas seguem uma lei exponencial com um parâmetro comum desconhecido $\lambda$.

Os métodos existentes para estimar $\mathrm{N}$ e $\lambda$ são baseados também na amostragem censurada, na qual o software é testado por um tempo fixo $\tau$ e os sucessivos tempos de falhas são observados.

\subsection{Objetivos}

O objetivo deste trabalho é estudar, do ponto de vista da teoria da verossimilhança e Bayesiana, o impacto da correção imperfeita $(p<1)$ sobre a 
informação adicional a respeito de $\mathrm{N}$ e $\lambda$ fornecida pelo contador $\mathrm{M}_{\mathrm{i}}$, e com um tempo total de teste fixado (censurado). E também comparar as distribuições a posteriori marginais de $\mathrm{N}$ para diferentes valores de $\mathrm{p}$, usando algumas medidas de divergência.

No capítulo 2, será detalhado o modelo de Jelinski e Moranda (1972). E, utilizando esse modelo, adicionaremos os contadores $M_{i}$ e mostraremos que esse modelo, chamado agora modelo de captura-recaptura, proposto por Nayak (1988), nos dá uma informação adicional a respeito de $\mathrm{N}$ e $\lambda$. Ainda neste capítulo, mostramos que, utilizando processos de Poisson, chegamos aos mesmos resultados do modelo de captura-recaptura de Nayak, com a vantagem de não precisarmos observar os tempos de falhas.

Também no capítulo 2, veremos o modelo de Goel e Okumoto (1978) e adicionaremos os contadores a esse modelo. Agora teremos o modelo de capturarecaptura com correção imperfeita $(\mathrm{p}<1)$. Estudaremos o caso com $\mathrm{p}$ conhecido e desconhecido e obteremos os estimadores de máxima verossimilhança de $N$, $\lambda$ e p.

No capítulo 3, utilizaremos inferência Bayesiana. Nos modelos acima, quando temos poucos dados de falhas do software, ocorrem problemas com o uso da estimativa de máxima verossimilhança para os parâmetros de interesse. Nesse caso, uma análise Bayesiana do modelo pode superar esse problema, devido à possibilidade de incorporar a opinião de um especialista (uma priori) no modelo. Tal opinião pode dar uma grande quantidade de informações relevantes neste caso. Com a priori e os dados de falhas, a posteriori pode ser obtida via teorema de 
Bayes. Utilizaremos também os algoritmos de Gibbs Sampling e MetropolisHastings para obtermos os resumos a posteriori das densidades marginais.

Veremos ainda no capítulo 3 que, quando usamos priori não-informativa, a densidade a posteriori pode não existir, mas ela sempre existirá se adicionarmos a esse modelo os contadores $\mathrm{M}_{\mathrm{i}}$. Daí a importância de incorporarmos os contadores $\mathrm{M}_{\mathrm{i}}$ ao modelo.

No capítulo 4, nós compararemos a distribuição a posteriori de $\mathrm{N}$, obtida no capítulo 3, para diferentes valores de p. O que queremos saber é se mudanças na probabilidade de corrigir um erro, quando este é encontrado, corresponde a mudanças substanciais nos resultados da análise. Iremos comparar as distribuições a posteriori de $\mathrm{N}, \pi$ e $\pi_{0}$, para diferentes valores de $\mathrm{p}$, usando as medidas de divergência de Kullback-Leibler (KL) e a Variação Total (VT).

Um exemplo de aplicação das metodologias descritas é desenvolvido no capítulo 5, utilizando-se um conjunto de dados.

Finalmente no capítulo 6, são apresentadas as conclusões e comentários sobre perspectivas futuras. 


\section{CAPÍTULO 2}

\section{ALGUNS MODELOS DE CONFIABILIDADE DE SOFTWARE}

\subsection{O Modelo de Jelinski e Moranda}

Jelinski e Moranda (1972) propuseram um modelo simples para descrever as falhas do software. Esse modelo foi o primeiro a ser amplamente utilizado e tem servido de base para muitos, desenvolvidos posteriormente.

Suponha-se que existem $\mathrm{N}$ erros no software no início do teste. Cada erro é independente dos outros e é igualmente provável causa de falhas durante o teste. Quando o software falha, o erro é corrigido com probabilidade 1, ou seja, a correção de um erro não ocasiona outro.

\subsubsection{Função de Verossimilhança}

Assumindo-se que os tempos de falhas $\mathrm{T}_{\mathrm{i}}$ são variáveis aleatórias exponenciais independentes com taxa de falha comum $\lambda$, e supondo-se censura do tipo II, onde somente os $\mathrm{r}$ primeiros tempos de falhas são observados, ou seja, $\mathrm{t}_{(1)} \leq \mathrm{t}_{(2)} \leq \ldots \leq \mathrm{t}_{(\mathrm{r})}$, a função de verossimilhança para $\mathrm{N}$ e $\lambda$ é dada por 


$$
\begin{aligned}
L\left(N, \lambda \mid t_{(1)}, t_{(2)}, \ldots, t_{(r)}\right) & =\frac{N !}{(N-r) !}\left(\prod_{i=1}^{r} \lambda e^{-\lambda t_{(i)}}\right)\left[e^{-\lambda t_{(r)}}\right]^{(N-r)} \\
& =\lambda^{r}\left(\prod_{i=1}^{r}(N-i+1)\right) \exp \left\{-\lambda\left[\sum_{i=1}^{r} t_{(i)}+(N-r) t_{(r)}\right]\right\} .
\end{aligned}
$$

A função de verossimilhança (2.1) também pode ser escrita através dos tempos entre falhas $\left(w_{i}\right)$. A relação dos tempos entre falhas, $\left(w_{i}\right)$, com os tempos de falhas, $\left(\mathrm{t}_{(\mathrm{i})}\right)$, é dada no teorema 1 .

TEOREMA 1: $\sum_{\mathrm{i}=1}^{\mathrm{r}}(\mathrm{N}-\mathrm{i}+1) \mathrm{w}_{\mathrm{i}}=\sum_{\mathrm{i}=1}^{\mathrm{r}} \mathrm{t}_{(\mathrm{i})}+(\mathrm{N}-\mathrm{r}) \mathrm{t}_{(\mathrm{r})}$

Prova:

$$
\begin{aligned}
& \sum_{i=1}^{r}(N-i+1) w_{i}=\sum_{i=1}^{r}(N-i+1)\left(t_{(i)}-t_{(i-1)}\right) \\
= & N t_{(1)}+(N-1)\left(t_{(2)}-t_{(1)}\right)+(N-2)\left(t_{(3)}-t_{(2)}\right)+\ldots+(N-r+1)\left(t_{(r)}-t_{(r-1)}\right) \\
= & t_{(1)}(N-N+1)+t_{(2)}(N-1-N+2)+\ldots+t_{(r-1)}(N-r+2-N+r-1)+t_{(r)}(N-r+1) \\
= & t_{(1)}+t_{(2)}+t_{(3)}+\ldots+t_{(r-1)}+t_{(r)}+(N-r) t_{(r)} \\
= & \sum_{i=1}^{r} t_{(i)}+(N-r) t_{(r)}
\end{aligned}
$$


Usando o teorema 1, podemos reescrever a função de verossimilhança (2.1) como

$$
\left.L(N, \lambda)=\lambda^{r}\left(\prod_{i=1}^{\mathrm{r}}(\mathrm{N}-\mathrm{i}+1)\right) \mathrm{e}^{\left\{-\lambda\left[\sum_{\mathrm{i}=1}^{\mathrm{r}}(\mathrm{N}-\mathrm{i}+1) \mathrm{w}_{\mathrm{i}}\right]\right.}\right\} .
$$

O logaritmo da função de verossimilhança (2.2) é dado por

$$
1(N, \lambda)=\sum_{i=1}^{r} \log (N-i+1)+r \log \lambda-\lambda \sum_{i=1}^{\mathrm{r}}(N-i+1) w_{i} .
$$

\subsubsection{Estimadores de Máxima Verossimilhança}

Fazendo as derivadas parciais de (2.3) e igualando a zero, teremos:

$$
\begin{aligned}
& \frac{\partial 1(N, \lambda)}{\partial N}=\sum_{i=1}^{r} \frac{1}{N-i+1}-\lambda \sum_{i=1}^{r} w_{i}=0, \\
& \frac{\partial 1(N, \lambda)}{\partial \lambda}=\frac{r}{\lambda}-\sum_{i=1}^{r}(N-i+1) w_{i}=0 .
\end{aligned}
$$

Resolvendo o sistema de equações em $\mathrm{N}$ e $\lambda$, e definindo $\mathrm{S}_{\mathrm{r}}=\sum_{\mathrm{i}=1}^{\mathrm{r}} \mathrm{w}_{\mathrm{i}}$, nós obtemos os estimadores de máxima verossimilhança de $\lambda$ e $\mathrm{N}$, dados por: 


$$
\hat{\lambda}=\frac{\mathrm{r}}{\hat{N S_{r}-\sum_{i=1}^{r}(i-1) w_{i}}}
$$

onde $\hat{\mathrm{N}}$ é a solução da equação,

$$
\sum_{i=1}^{r} \frac{1}{N-i+1}=\frac{r S_{r}}{N S_{r}-\sum_{i=1}^{r}(i-1) w_{i}}=\frac{r}{N-\frac{1}{S_{r}} \sum_{i=1}^{r}(i-1) w_{i}}
$$

Devemos observar que, para a obtenção do e.m.v. de N, necessitamos do uso de um método iterativo, por exemplo, o método de Newton (ver apêndice 1), pois a equação (2.7) é não linear e $\hat{\mathrm{N}}$ não pode ser obtido explicitamente.

\subsubsection{Função de Verossimilhança Profile}

A função de verossimilhança profile $L_{p}(N)$ é dada por

$$
\mathrm{L}_{\mathrm{p}}(\mathrm{N})=\mathrm{L}(\mathrm{N}, \hat{\lambda}(\mathrm{N}))=\left(\mathrm{r}^{-1} \sum_{\mathrm{i}=1}^{\mathrm{r}}(\mathrm{N}-\mathrm{i}+1) \mathrm{w}_{\mathrm{i}}\right)^{\mathrm{r}}\left(\prod_{\mathrm{i}=1}^{\mathrm{r}}(\mathrm{N}-\mathrm{i}+1)\right)
$$

Considere-se $\mathrm{g}(\mathrm{N})=\mathrm{L}_{\mathrm{p}}(\mathrm{N}) / \mathrm{L}_{\mathrm{p}}(\mathrm{N}-1)$. Com esta razão estamos analisando a função $L_{p}(N)$ passo a passo, e, dessa maneira, o estimador de máxima verossimilhança de $\mathrm{N}$ será $\hat{\mathrm{N}}_{\mathrm{p}}=\mathrm{r}$ se $\mathrm{g}(\mathrm{r})>1$ e $\mathrm{g}(\mathrm{r}+1)<1 . \operatorname{Se} \mathrm{g}(\mathrm{r}+1)>1$, $\hat{\mathrm{N}}_{\mathrm{p}}=\max \{\mathrm{N}>\mathrm{r}: \mathrm{g}(\mathrm{N})>1\}$, onde 


$$
g(N)=\frac{L_{p}(N)}{L_{p}(N-1)}=\frac{\left(r^{-1} \sum_{i=1}^{r}(N-i+1) w_{i}\right)^{r}\left(\prod_{i=1}^{r}(N-i+1)\right)}{\left(r^{-1} \sum_{i=1}^{r}(N-i) w_{i}\right)^{r}\left(\prod_{i=1}^{r}(N-i)\right)}
$$

Para este modelo, o estimador de máxima verossimilhança de $\mathrm{N}, \hat{\mathrm{N}}$, é o mesmo que maximiza $L_{p}(N)$.

\subsubsection{Inferências sobre os Parâmetros do Modelo}

A partir dos estimadores de máxima verossimilhança $\hat{N}$ e $\hat{\lambda}$, podemos obter intervalos de confiança e testes de hipóteses para os parâmetros do modelo, usando a teoria assintótica para grandes amostras baseada na matriz de informação de Fisher ou informação observada (ver por exemplo, Bickel e Doksum, 1977). Assim, consideramos

$$
(\hat{N}, \hat{\lambda}) \stackrel{a}{\sim} \mathrm{N}\left\{(\mathrm{N}, \lambda) ; \mathrm{I}^{-1}(\hat{\mathrm{N}}, \hat{\lambda})\right\}
$$

onde $\mathrm{I}(\mathrm{N}, \lambda)$ é a matriz de informação de Fisher dada por

$$
I(N, \lambda)=\left[\begin{array}{ll}
E\left\{\frac{-\partial^{2} 1(N, \lambda)}{\partial N^{2}}\right\} & E\left\{\frac{-\partial^{2} 1(N, \lambda)}{\partial N \partial \lambda}\right\} \\
E\left\{\frac{-\partial^{2} 1(N, \lambda)}{\partial N \partial \lambda}\right\} & E\left\{\frac{-\partial^{2} 1(N, \lambda)}{\partial \lambda^{2}}\right\}
\end{array}\right]
$$


Assim,

$$
I(N, \lambda)=\left[\begin{array}{cc}
\sum_{i=1}^{\mathrm{r}}\left[\frac{1}{(\mathrm{~N}-\mathrm{i}+1)^{2}}\right] & \frac{1}{\lambda} \sum_{\mathrm{i}=1}^{\mathrm{r}}\left[\frac{1}{(\mathrm{~N}-\mathrm{i}+1)}\right] \\
\frac{1}{\lambda} \sum_{\mathrm{i}=1}^{\mathrm{r}}\left[\frac{1}{(\mathrm{~N}-\mathrm{i}+1)}\right] & \frac{\mathrm{r}}{\lambda^{2}}
\end{array}\right]
$$

e $\mathrm{I}^{-1}(\mathrm{~N}, \lambda)$, o inverso da matriz de informação de Fisher, dada em (2.11), representa a matriz de variância e covariância assintótica de $\hat{N}$ e $\hat{\lambda}$.

Considerando a distribuição Normal assintótica dos e.m.v., podemos construir intervalos de confiança para os parâmetros $\mathrm{N}$ e $\lambda$.

Os intervalos de confiança para $\mathrm{N}$ e $\lambda$, considerando-se um nível de confiança $100(1-\alpha) \%$, são dados por:

$$
\begin{aligned}
& \mathrm{P}\left[\hat{\mathrm{N}}-\mathrm{z}_{\alpha / 2} \sqrt{\mathrm{I}_{11}^{-1}(\mathrm{~N}, \lambda)} \leq \mathrm{N} \leq \hat{\mathrm{N}}+\mathrm{z}_{\alpha / 2} \sqrt{\mathrm{I}_{11}^{-1}(\mathrm{~N}, \lambda)}\right] \cong 1-\alpha, \\
& \mathrm{P}\left[\hat{\lambda}-\mathrm{z}_{\alpha / 2} \sqrt{\mathrm{I}_{22}^{-1}(\mathrm{~N}, \lambda)} \leq \lambda \leq \hat{\lambda}+\mathrm{z}_{\alpha / 2} \sqrt{\mathrm{I}_{22}^{-1}(\mathrm{~N}, \lambda)}\right] \cong 1-\alpha,
\end{aligned}
$$

onde $\mathrm{I}_{\mathrm{ii}}^{-1}(\mathrm{~N}, \lambda), \mathrm{i}=1,2$ representa o i-ésimo elemento diagonal da matriz de variância e covariância assintótica de $\hat{\mathrm{N}}$ e $\hat{\lambda}$, e $\mathrm{z}_{\alpha / 2}$ é um percentil da distribuição normal padronizada obtido de uma tabela estatística. 


\subsection{O Modelo de Captura-Recaptura de Nayak}

Um planejamento chamado correção por recaptura é proposto por Nayak (1988) para obter informação extra que estime o número original de erros, N, em um sistema de confiabilidade. O sistema é observado por um período fixo de tempo $\tau$ e assume-se que as falhas seguem uma lei exponencial com um parâmetro comum desconhecido $\lambda$.

Considere-se um pedaço de software do computador com um número de erros desconhecidos N. Quando o software é executado, um particular caminho no software é seguido e o software falha se, e somente se, um dos erros é encontrado naquele caminho. Um problema importante no desenvolvimento do software é a estimação de $\mathrm{N}$ (números de erros existentes no software).

O modelo mais utilizado para descrever as falhas de um software é o proposto por Jelinski e Moranda (1972). Nesse modelo, os tempos de deteç̧ão dos erros, $T_{i}$, são variáveis exponenciais independentes com uma taxa de falha comum $\lambda$, e quando o software falha, o erro causador da falha é detectado e removido sem a inserção de erros adicionais.

Os métodos existentes para estimar $\mathrm{N}$ e $\lambda$ são baseados também na amostragem censurada, na qual o software é testado por um tempo fixo $\tau$ e os sucessivos tempos de falhas $\mathrm{t}_{(1)} \leq \mathrm{t}_{(2)} \leq \ldots \leq \mathrm{t}_{(\mathrm{r})} \leq \tau$ são observados ( $\mathrm{r}$ é uma variável aleatória). 
As suposições de Jelinski e Moranda (1972) podem não ser apropriadas em algumas aplicações; e modelos alternativos têm sido propostos. Contudo, o modelo de Jelinski e Moranda é básico no tópico confiabilidade de software, e desse modo, suas suposições básicas serão adotadas.

\subsubsection{Correção por Recaptura}

Informação extra sobre $\lambda$ pode ser obtida pela não remoção dos erros e observando-se as freqüências dos erros detectados em adição aos primeiros tempos de detecção. É claro que seria inconveniente ao usuário admitir um erro sobrando no sistema. Em particular, quando um erro é removido, nós podemos inserir um contador no software para contar o número de vezes que o erro poderia ter ocorrido nos tempos restantes do teste.

Para especificar, suponha-se que o software contenha inicialmente $\mathrm{N}$ erros em áreas desconhecidas $A_{1}, \ldots, A_{N}$. Quando um erro em $A_{J}$ é detectado, ele é corrigido, e um contador adicionado ao software guarda o número de vezes, $M_{J}$, que $A_{J}$ é acessado novamente. Seguindo esse procedimento, nós podemos observar $\mathrm{V}=\left(\mathrm{M}_{1}, \ldots, \mathrm{M}_{\mathrm{r}}\right)$ em adição a $\mathrm{U}=\left(\mathrm{r}, \mathrm{T}_{(1)}, \ldots, \mathrm{T}_{(\mathrm{r})}\right)$.

Como no modelo de Jelinski e Moranda, nós assumimos que os erros são acessados de acordo com processos de Poisson homogêneos independentes; porém, nós permitiremos acessos repetidos. Seja $\mathrm{X}_{\mathrm{J}}(\mathrm{t})$ o número de ocorrências do j-ésimo erro no tempo t. Assim, $X_{J}(t)(j=1, \ldots, N)$ são processos de Poisson homogêneos independentes com uma taxa comum $\lambda$. 


\subsubsection{Função de Verossimilhança}

Sobre o modelo, dado $\left\{\mathrm{t}_{(\mathrm{i})}\right\}$, os tempos de falhas, $\left\{\mathrm{M}_{\mathrm{i}}\right\}$ são variáveis aleatórias de Poisson independentes com parâmetros $\left\{\lambda\left(\tau-\mathrm{t}_{(\mathrm{i})}\right)\right\}$ e a função de verossimilhança considerados $\mathrm{u}=\left(\mathrm{r}, \mathrm{t}_{(1)}, \ldots, \mathrm{t}_{(\mathrm{r})}\right)$ e $\mathrm{v}=\left(\mathrm{m}_{1}, \ldots, \mathrm{m}_{\mathrm{r}}\right)$ é dada por

$$
\begin{aligned}
& L_{u, v}(N, \lambda)=p(u ; \lambda, N) p_{v \mid u}(v ; \lambda, N \mid u) \\
& =\frac{N !}{(N-r) !} \lambda^{r} \exp \left[-\lambda\left\{(N-r) \tau+\sum_{i=1}^{r} t_{(i)}\right\}\right] \frac{\lambda^{m}}{\prod_{i=1}^{r} m_{i} !} \prod_{i=1}^{r}\left(\tau-t_{(i)}\right)^{m_{i}} \exp \left\{-\lambda\left(r \tau-\sum_{i=1}^{r} t_{(i)}\right)\right\} \\
& =\frac{N !}{(N-r) ! \prod_{i=1}^{r} m_{i} !} \lambda^{r+m} \exp \left[-\lambda N \tau+\lambda r \tau-\lambda \sum_{i=1}^{r} t_{(i)}-\lambda r \tau+\lambda \sum_{i=1}^{r} t_{(i)}\right] \prod_{i=1}^{r}\left(\tau-t_{(i)}\right)^{m_{i}} \\
& =\frac{N ! \lambda^{m+r} \exp (-N \lambda \tau) \prod_{i=1}^{r}\left(\tau-t_{(i)}\right)^{m_{i}}}{(N-r) ! \prod_{i=1}^{r} m_{i} !} \\
& =\frac{N !}{(N-r) !} \lambda^{m+r} \exp (-N \lambda \tau) G(m, r, t, \tau) .
\end{aligned}
$$

Observe-se que se não adicionarmos os contadores, teremos a função de verossimilhança (2.1) do modelo de Jelinski e Moranda. 
Como $\mathrm{G}(\mathrm{m}, \mathrm{r}, \mathrm{t}, \tau)$ não dá alguma informação a respeito de $\mathrm{N}$ e $\lambda$, podemos considerar a função de verossimilhança (2.14) como sendo

$$
\mathrm{L}(\mathrm{N}, \lambda) \propto \frac{\mathrm{N} !}{(\mathrm{N}-\mathrm{r}) !} \lambda^{\mathrm{m}+\mathrm{r}} \exp (-\mathrm{N} \lambda \tau), \text { onde } \mathrm{m}=\sum_{\mathrm{i}=1}^{\mathrm{r}} \mathrm{m}_{\mathrm{i}}
$$

Note-se que, neste modelo, não é necessário observarmos os tempos de falhas $\left\{\mathrm{t}_{(\mathrm{i})}\right\}$. Somente o número total de falhas $(\mathrm{R}+\mathrm{M})$ e o número de erros $(\mathrm{R})$ causando essas falhas são relevantes.

\subsubsection{Estimadores de Máxima Verossimilhança}

Vamos assumir $r \geq 1$. Fazendo $1(N, \lambda)=\log [L(N, \lambda)]$ e derivando em relação a $\mathrm{N}$ e $\lambda$, teremos:

$$
\begin{aligned}
& \frac{\partial \mathrm{l}(\mathrm{N}, \lambda)}{\partial \mathrm{N}}=\sum_{\mathrm{i}=1}^{\mathrm{r}} \frac{1}{\mathrm{~N}-\mathrm{i}+1}-\lambda \tau=0, \\
& \frac{\partial \mathrm{l}(\mathrm{N}, \lambda)}{\partial \lambda}=\frac{\mathrm{m}+\mathrm{r}}{\lambda}-\mathrm{N} \tau=0 .
\end{aligned}
$$

O e.m.v. de $\mathrm{N}$ é a solução da equação

$$
\sum_{i=1}^{r} \frac{1}{N-i+1}=\frac{(r+m)}{N}
$$


Para solucionarmos numericamente a equação (2.18), podemos recorrer a métodos iterativos, como por exemplo, o de Newton (ver apêndice 1).

Tendo obtido $\hat{\mathrm{N}}$, o e.m.v. de $\lambda$ é dado por

$$
\hat{\lambda}=\frac{\mathrm{r}+\mathrm{m}}{\hat{\mathrm{N}} \tau}
$$

\subsubsection{Função de Verossimilhança Profile}

Para obter a função de verossimilhança profile de $\mathrm{N}$, basta substituir $\lambda=[(\mathrm{r}+\mathrm{m}) / \mathrm{N} \tau]$ na função de verossimilhança $(2.15) \mathrm{e}$, então, teremos que

$$
L_{p}(N)=\frac{N !}{(N-r) ! N^{m+r}}\left(\frac{r+m}{\tau}\right)^{m+r} e^{-(m+r)} .
$$

Como estamos interessados em maximizar $\mathrm{L}_{\mathrm{p}}(\mathrm{N})$ em relação a $\mathrm{N}$, podemos considerar a função de verossimilhança profile (2.20) como sendo

$$
\mathrm{L}_{\mathrm{p}}(\mathrm{N})=\frac{\mathrm{N}(\mathrm{N}-1) \ldots(\mathrm{N}-\mathrm{r}+1)}{\mathrm{N}^{\mathrm{m}+\mathrm{r}}}
$$

Se $m=0$, então $L_{p}(N)$ está crescendo em $N$ e $\hat{N}_{p}=\infty$. Se $m>0$, então $\hat{N}_{p}$ é determinado por 


$$
\begin{aligned}
& \frac{L_{p}(N)}{L_{p}(N-1)}=\frac{N(N-1)^{m+r}}{(N-r) N^{m+r}}=\left(\frac{N-1}{N}\right)^{m+r}\left(\frac{N}{N-r}\right), N>r, \\
& g(N)=\frac{L_{p}(N)}{L_{p}(N-1)}>1 \Leftrightarrow\left(1-\frac{1}{N}\right)^{m+r}>\left(1-\frac{r}{N}\right) \Leftrightarrow \\
& g(N)=\left(1-\frac{1}{N}\right)^{m+r}+\frac{r}{N}>1,
\end{aligned}
$$

porque $\mathrm{L}_{\mathrm{p}}(\mathrm{N})>\mathrm{L}_{\mathrm{p}}(\mathrm{N}-1)$ se, e somente se, $\mathrm{g}(\mathrm{N})>1$.

Se $g(r+1)<1, L_{p}(N)$ está decrescendo em $N$ e $\hat{N}_{p}=r$. Se $g(r+1)>1$, então $\hat{\mathrm{N}}_{\mathrm{p}}=\max \{\mathrm{N}>\mathrm{r}: \mathrm{g}(\mathrm{N})>1\}$. De (2.22) segue que, dado $\mathrm{R}=\mathrm{r}, \hat{\mathrm{N}}_{\mathrm{p}}$ é uma função decrescente de $\mathrm{me} \hat{\mathrm{N}}_{\mathrm{p}}=\mathrm{k}$ se, e somente $\mathrm{se}, \mathrm{h}_{\mathrm{r}, \mathrm{k}+1}<\mathrm{m}<\mathrm{h}_{\mathrm{r}, \mathrm{k}}$, onde

$$
\mathrm{h}_{\mathrm{r}, \mathrm{k}}=\frac{\log \left(1-\frac{\mathrm{r}}{\mathrm{k}}\right)}{\log \left(1-\frac{1}{\mathrm{k}}\right)}-\mathrm{r}, \mathrm{k}>\mathrm{r} \text {. }
$$

e $h_{r, r}=\infty$. A seguir, veremos como foi obtida a expressão em (2.23).

Quando $\mathrm{g}(\mathrm{r}+1)>1$, temos que $\hat{\mathrm{N}}_{\mathrm{p}}=\max \{\mathrm{N}>\mathrm{r}: \mathrm{g}(\mathrm{N})>1\}$. Se $\hat{\mathrm{N}}_{\mathrm{p}}=\mathrm{k}(\mathrm{k}>\mathrm{r})$, então $k$ é o maior valor no qual $g(k)>1$ e, conseqüentemente, $g(k+1)<1$. Dessa maneira, teremos 


$$
\begin{aligned}
\mathrm{g}(\mathrm{k})>1 & \Rightarrow\left(\frac{\mathrm{k}-1}{\mathrm{k}}\right)^{\mathrm{m}+\mathrm{r}}+\frac{\mathrm{r}}{\mathrm{k}}>1 \Rightarrow\left(1-\frac{1}{\mathrm{k}}\right)^{\mathrm{m}+\mathrm{r}}>1-\frac{\mathrm{r}}{\mathrm{k}} \\
& \Rightarrow(\mathrm{m}+\mathrm{r}) \log \left(1-\frac{1}{\mathrm{k}}\right)>\log \left(1-\frac{\mathrm{r}}{\mathrm{k}}\right) \\
& \Rightarrow(\mathrm{m}+\mathrm{r})<\frac{\log \left(1-\frac{\mathrm{r}}{\mathrm{k}}\right)}{\log \left(1-\frac{1}{\mathrm{k}}\right)} \Rightarrow \mathrm{m}<\frac{\log \left(1-\frac{\mathrm{r}}{\mathrm{k}}\right)}{\log \left(1-\frac{1}{\mathrm{k}}\right)}-\mathrm{r} \Rightarrow \mathrm{m}<\mathrm{h}_{\mathrm{r}, \mathrm{k}}
\end{aligned}
$$

De modo análogo, $g(k+1)<1 \Rightarrow m>h_{r, k+1}$. Portanto, $\hat{\mathrm{N}}_{\mathrm{p}}=\mathrm{k}$ se, e somente se, $\mathrm{h}_{\mathrm{r}, \mathrm{k}+1}<\mathrm{m}<\mathrm{h}_{\mathrm{r}, \mathrm{k}}$.

\subsubsection{Inferências sobre os Parâmetros do Modelo}

Para obtermos inferências sobre os parâmetros do modelo, utilizamos a aproximação Normal assintótica dos e.m.v., baseada na matriz de informação de Fisher ou informação observada. Assim consideramos

$$
(\hat{\mathrm{N}}, \hat{\lambda}) \stackrel{\mathrm{a}}{\sim} \mathrm{N}\left\{(\mathrm{N}, \lambda) ; \mathrm{I}_{0}^{-1}(\hat{\mathrm{N}}, \hat{\lambda})\right\}
$$

onde $\mathrm{I}_{0}(\mathrm{~N}, \lambda)$ é a matriz de informação observada, dada por 


$$
I_{0}(N, \lambda)=-\left[\begin{array}{ll}
\frac{\partial^{2} 1(N, \lambda)}{\partial N^{2}} & \frac{\partial^{2} 1(N, \lambda)}{\partial N \partial \lambda} \\
\frac{\partial^{2} 1(N, \lambda)}{\partial N \partial \lambda} & \frac{\partial^{2} 1(N, \lambda)}{\partial \lambda^{2}}
\end{array}\right]
$$

Assim,

$$
I_{0}(N, \lambda)=\left[\begin{array}{cc}
\sum_{i=1}^{r}\left[\frac{1}{(N-i+1)^{2}}\right] & \tau \\
\tau & \frac{m+r}{\lambda^{2}}
\end{array}\right]
$$

e $\mathrm{I}_{0}^{-1}(\mathrm{~N}, \lambda)$, o inverso da matriz de informação observada, dada em (2.25), representa a matriz de variância e covariância assintótica de $\hat{\mathrm{N}}$ e $\hat{\lambda}$.

Considerando a distribuição Normal assintótica dos e.m.v., podemos construir intervalos de confiança para os parâmetros $\mathrm{N}$ e $\lambda$.

Os intervalos de confiança para $\mathrm{N}$ e $\lambda$, considerando-se um nível de confiança $100(1-\alpha) \%$, são dados por:

$$
\begin{aligned}
& \mathrm{P}\left[\hat{\mathrm{N}}-\mathrm{z}_{\alpha / 2} \sqrt{\mathrm{I}_{0}^{-1}{ }_{11}^{-1}(\mathrm{~N}, \lambda)} \leq \mathrm{N} \leq \hat{\mathrm{N}}+\mathrm{z}_{\alpha / 2} \sqrt{\mathrm{I}_{0_{11}^{-1}}^{-1}(\mathrm{~N}, \lambda)}\right] \cong 1-\alpha, \\
& \mathrm{P}\left[\hat{\lambda}-\mathrm{z}_{\alpha / 2} \sqrt{\mathrm{I}_{0}{ }_{22}^{-1}(\mathrm{~N}, \lambda)} \leq \lambda \leq \hat{\lambda}+\mathrm{z}_{\alpha / 2} \sqrt{\mathrm{I}_{0_{22}^{-1}(\mathrm{~N}, \lambda)}^{-1}}\right] \cong 1-\alpha,
\end{aligned}
$$


onde $\mathrm{I}_{0_{\mathrm{ii}}}^{-1}(\mathrm{~N}, \lambda), \mathrm{i}=1,2$ representa o $\mathrm{i}$-ésimo elemento diagonal da matriz de variância e covariância assintótica de $\hat{\mathrm{N}}$ e $\hat{\lambda}$, e $\mathrm{z}_{\alpha / 2}$ é um percentil da distribuição normal padronizada obtido de uma tabela estatística.

Um fato importante a ser notado é que, embora os tempos de falhas tenham sido observados, eles não trazem informação adicional para os estimadores de máxima verossimilhança de $\mathrm{N}$ e $\lambda$. Uma alternativa para esse problema, que será vista a seguir, seria utilizar processos de Poisson.

\subsubsection{Processos de Poisson}

O processo de Poisson oferece uma boa aproximação a muitas situações do mundo real. Uma lista de casos onde esse processo tem provado ser de utilidade seria muito extensa. As chamadas telefônicas, encomendas dirigidas a uma fábrica,chegadas a uma fila, reclamações de seguros, ocorrência de epidemias, etc., são apenas alguns exemplos (ver Clarke e Disney, 1970).

Seja $X(\tau, \tau+\Delta \tau)$ denotando o número de eventos ocorridos em um intervalo de tempo $(\tau, \tau+h)$, onde $\tau \geq 0$ e $\Delta \tau>0$. Este processo é um processo de Poisson se

$$
\begin{aligned}
& \mathrm{P}[\mathrm{X}(\tau, \tau+\Delta \tau)=0]=1-\lambda \Delta \tau+\mathrm{o}(\Delta \tau), \\
& \mathrm{P}[\mathrm{X}(\tau, \tau+\Delta \tau)=1]=\lambda \Delta \tau+\mathrm{o}(\Delta \tau),
\end{aligned}
$$


com $\Delta \tau \rightarrow 0$, onde $\lambda>0$ é uma constante, chamada a intensidade do processo, e o(h) denota a quantidade $\mathrm{g}(\mathrm{h})$ para a qual $\lim _{\Delta \tau \rightarrow 0} \mathrm{O}(\Delta \tau) / \Delta \tau=0$.

As condições acima implicam que $\mathrm{P}[\mathrm{X}(\tau, \tau+\Delta \tau) \geq 2]=\mathrm{o}(\Delta \tau)$ e que a ocorrência de eventos em $(\tau, \tau+\Delta \tau)$ não é afetada pelos acontecimentos anteriores ao tempo $\tau$. Ou seja, a probabilidade de ocorrência num intervalo pequeno $\Delta \tau$ é proporcional ao comprimento do intervalo e não depende da posição, e as ocorrências dos eventos em intervalos distintos são independentes, e a probabilidade de dois ou mais eventos num intervalo $\Delta \tau$ pequeno é desprezível.

De (2.28) e (2.29) pode ser mostrado que para algum $s \geq 0, \tau>0$,

$$
\mathrm{P}_{\mathrm{n}}(\tau)=\mathrm{P}[\mathrm{X}(\mathrm{s}, \mathrm{s}+\tau)=\mathrm{n}]=\mathrm{e}^{-\lambda \tau} \frac{(\lambda \tau)^{\mathrm{n}}}{\mathrm{n} !} \quad \mathrm{n}=0,1,2, \ldots
$$

a qual é uma distribuição de Poisson com média $\lambda \tau$ e variância $\lambda \tau$. O processo é dito homogêneo porque (2.30) não depende de s.

\subsubsection{Obtendo o Modelo de Captura-Recaptura via Processos de Poisson}

Estamos interessados em estimar $\mathrm{N}$ após observar o software durante um intervalo de tempo $[0, \tau], \tau>0$ ( $\tau$ é dado).

Dados $\mathrm{N}$ e $\lambda>0$, cada região $\mathrm{A}_{\mathrm{J}}$ é acessada ou percorrida durante a execução do programa, independentemente das demais, após tempos aleatórios exponenciais 
independentes de parâmetro $\lambda: \mathrm{P}\left[\mathrm{T}_{\mathrm{J}} \geq \tau\right]=\mathrm{e}^{-\lambda \tau}, \tau>0$. Observado um erro em qualquer região, o mesmo é corrigido com a introdução de um contador nessa região.

Seja $X_{J}(\tau)$ o número de vezes em que a região $A_{J}, j=1,2, \ldots, N$, é percorrida (acessada) pelo programa (incluindo a primeira chegada), durante o intervalo de tempo $[0, \tau] . \mathrm{X}_{\mathrm{J}}(\tau)$ é um processo de Poisson com taxa $\lambda \tau$.

Considere-se agora $N_{\tau}$ o número de vezes em que as regiões $A_{1}, A_{2}, \ldots, A_{N}$ são percorridas pelo programa, durante o intervalo de tempo $[0, \tau]$.

$$
\mathrm{N}_{\tau}=\mathrm{X}_{1}(\tau)+\mathrm{X}_{2}(\tau)+\mathrm{X}_{3}(\tau)+\ldots+\mathrm{X}_{\mathrm{N}}(\tau)
$$

$\mathrm{N}_{\tau}$ é observável, isto é, é uma estatística e tem uma distribuição de Poisson com taxa $N \lambda \tau$.

Vamos supor agora que:

$$
\begin{gathered}
E_{J}(\tau)=\left\{\begin{array}{l}
1, \text { se a região } j \text { for percorrida pelo programa durante o intervalo }[0, \tau] \\
0, \text { caso contrário. }
\end{array}\right. \\
\qquad\left[E_{J}(\tau)=0\right]=P\left[X_{J}(\tau)=0\right]=e^{-\lambda \tau} \\
P\left[E_{J}(\tau)=1\right]=P\left[X_{J}(\tau)>0\right]=1-e^{-\lambda \tau}
\end{gathered}
$$


Considere $\mathrm{W}_{\tau}$ o número de erros observados durante o intervalo $[0, \tau]$,

$$
\mathrm{W}_{\tau}=\mathrm{E}_{1}(\tau)+\mathrm{E}_{2}(\tau)+\mathrm{E}_{3}(\tau)+\ldots+\mathrm{E}_{\mathrm{N}}(\tau)
$$

$\mathrm{W}_{\tau}$ tem uma distribuição binomial com parâmetros $\mathrm{N}$ e $\mathrm{p}=1-\mathrm{e}^{-\lambda \tau}, \mathrm{e} \mathrm{N}_{\tau}$ tem uma distribuição de Poisson com parâmetro $N \lambda \tau$, ou seja,

$$
\begin{aligned}
& \mathrm{P}\left(\mathrm{W}_{\tau}=\mathrm{w} \mid \mathrm{N}, \lambda\right)=\left(\begin{array}{c}
\mathrm{N} \\
\mathrm{w}
\end{array}\right)\left(1-\mathrm{e}^{-\lambda \tau}\right)^{\mathrm{w}}\left(\mathrm{e}^{-\lambda \tau}\right)^{\mathrm{N}-\mathrm{w}}, \quad 0 \leq \mathrm{w} \leq \mathrm{N}, \\
& \mathrm{P}\left(\mathrm{N}_{\tau}=\mathrm{n} \mid \mathrm{N}, \lambda\right)=\mathrm{e}^{-\mathrm{N} \lambda \tau} \frac{(\mathrm{N} \lambda \tau)^{\mathrm{n}}}{\mathrm{n} !}, \mathrm{n}=0,1,2, \ldots
\end{aligned}
$$

Quando não colocamos os contadores, temos só as primeiras chegadas. Nesse caso, $\mathrm{W}_{\tau}$ é a estatística e então

$$
\mathrm{L}(\mathrm{N}, \lambda \mid \mathrm{w})=\left(\begin{array}{l}
\mathrm{N} \\
\mathrm{w}
\end{array}\right)\left(1-\mathrm{e}^{-\lambda \tau}\right)^{\mathrm{w}}\left(\mathrm{e}^{-\lambda \tau}\right)^{\mathrm{N}-\mathrm{w}}, \mathrm{N} \geq \mathrm{w}, \lambda>0
$$

Com os contadores, $\left(\mathrm{W}_{\tau}, \mathrm{N}_{\tau}\right)$ é a estatística. Dados $\mathrm{N}$ e $\lambda$, a distribuição de $\left(\mathrm{W}_{\tau}, \mathrm{N}_{\tau}\right)$ é dada por

$$
\mathrm{P}\left(\mathrm{W}_{\tau}=\mathrm{w}, \mathrm{N}_{\tau}=\mathrm{n} \mid \mathrm{N}, \lambda\right)=\sum_{\mathrm{x}_{1}, \mathrm{x}_{2}, \ldots, \mathrm{x}_{\mathrm{N}}} \prod_{\mathrm{i}=1}^{\mathrm{N}} \frac{\mathrm{e}^{-\lambda \tau}(\lambda \tau)^{\mathrm{x}_{\mathrm{i}}}}{\mathrm{x}_{\mathrm{i}} !}
$$




$$
\begin{aligned}
& =\sum_{\mathrm{x}_{1}, \mathrm{x}_{2}, \ldots, \mathrm{x}_{N}} \frac{\mathrm{e}^{-\mathrm{N} \lambda \tau}(\lambda \tau)^{\sum_{i=1}^{N} \mathrm{x}_{\mathrm{i}}}}{\prod_{\mathrm{i}=1}^{\mathrm{N}} \mathrm{x}_{\mathrm{i}} !}=\mathrm{e}^{-\mathrm{N} \lambda \tau}(\lambda \tau)^{\mathrm{n}} \sum_{\mathrm{x}_{1}, \mathrm{x}_{2}, \ldots, \mathrm{x}_{\mathrm{N}}} \frac{1}{\left(\prod_{\mathrm{i}=1}^{N} \mathrm{x}_{\mathrm{i}} !\right)} \\
& =\left(\begin{array}{l}
\mathrm{N} \\
\mathrm{w}
\end{array}\right) \mathrm{e}^{-\mathrm{N} \lambda \tau}(\lambda \tau)^{\mathrm{n}} \sum_{\substack{\mathrm{x}_{1}>0, \mathrm{x}_{2}>0, \ldots, \mathrm{x}_{\mathrm{w}}>0 \\
\mathrm{x}_{1}+\mathrm{x}_{2}+\ldots+\mathrm{x}_{\mathrm{w}}=\mathrm{n}}} \frac{1}{\left(\prod_{\mathrm{i}=1}^{\mathrm{N}} \mathrm{x}_{\mathrm{i}} !\right)} \\
& =\frac{N !}{(N-w) !} e^{-N \lambda \tau}(\lambda)^{n} G(w, n, \tau), n=1,2, \ldots \text { e } 1 \leq w \leq n \wedge N
\end{aligned}
$$

e

$$
\mathrm{P}\left(\mathrm{W}_{\tau}=0, \mathrm{~N}_{\tau}=0 \mid \mathrm{N}, \lambda\right)=\mathrm{P}\left(\mathrm{N}_{\tau}=0 \mid \mathrm{N}, \lambda\right)=\mathrm{e}^{-\mathrm{N} \lambda \tau}
$$

Como $\mathrm{G}(\mathrm{w}, \mathrm{n}, \tau)$ em (2.38) não dá alguma informação sobre $\mathrm{N}$, teremos

$$
\mathrm{L}(\mathrm{N}, \lambda) \propto \frac{\mathrm{N} !}{(\mathrm{N}-\mathrm{w}) !} \mathrm{e}^{-\mathrm{N} \lambda \tau}(\lambda)^{\mathrm{n}}
$$

e o estimador de máxima verossimilhança de $\mathrm{N}$ e $\lambda$ é o ponto de máximo de $\mathrm{L}(\mathrm{N}, \lambda)$. Se fizermos $n=(m+r)$ e $w=r$, teremos que a expressão (2.40) é idêntica à (2.15). 


\subsubsection{Estimação de $\mathrm{N}$ e $\lambda$}

Se $\mathrm{n}=0$ em (2.40), então $\mathrm{L}(\mathrm{N}, \lambda)=\mathrm{e}^{-\mathrm{N} \lambda \tau}, \mathrm{N} \geq 1, \lambda>0$. Para todo $\mathrm{N} \geq 1, \mathrm{~N}$ fixado, $\mathrm{L}(\mathrm{N}, \lambda)=\mathrm{e}^{-\mathrm{N} \lambda \tau}, \lambda \in[0, \infty)$, é tal que $\mathrm{L}(\mathrm{N}, 0)=1$ e $\mathrm{h}(\mathrm{N}, \lambda) \downarrow 0$, quando $\lambda \rightarrow \infty$. Logo, não existe $(\hat{\mathrm{N}}, \hat{\lambda})$ se $\mathrm{n}=0$.

Se $n \geq 1$ em (2.40), teremos

$$
L(N, \lambda)=\frac{N !}{(N-w) !} e^{-N \lambda \tau}(\lambda)^{n}, N \geq w, \lambda \in[0, \infty)
$$

Para cada $\mathrm{N} \geq \mathrm{w}, \mathrm{N}$ fixado, $\mathrm{L}(\mathrm{N}, 0)=0 \mathrm{e}$

$$
\begin{aligned}
& L^{\prime}(N, \lambda)=\frac{\partial \mathrm{L}(\mathrm{N}, \lambda)}{\partial \lambda}=\frac{\mathrm{N} !}{(\mathrm{N}-\mathrm{w}) !} \mathrm{e}^{-\mathrm{N} \lambda \tau} \lambda^{(\mathrm{n}-1)}(\mathrm{n}-\mathrm{N} \lambda \tau), \lambda \in(0, \infty) \\
\Rightarrow & \mathrm{L}^{\prime}(\mathrm{N}, \lambda)>0 \Leftrightarrow 0<\lambda<\mathrm{n} / \mathrm{N} \tau .
\end{aligned}
$$$$
\text { Logo, } \hat{\lambda}=\frac{\mathrm{n}}{\hat{\mathrm{N}} \tau} \text { e } \hat{\mathrm{N}} \text { é o ponto de máximo de }
$$$$
L_{P}\left(N, \frac{n}{N \tau}\right)=\frac{N !}{(N-w) !} e^{-N \tau\left(\frac{n}{N \tau}\right)}\left(\frac{n}{N \tau}\right)^{n}=\frac{N !}{(N-w) ! N^{n}} e^{-n}\left(\frac{n}{\tau}\right)^{n}, N \geq w,
$$

ou equivalentemente, $\hat{\mathrm{N}}$ é ponto de máximo de 


$$
L_{P}(N)=\frac{N !}{(N-w) ! N^{n}}, N \geq w
$$

onde $L_{P}(N)$ é a verossimilhança profile. Se $w=r$ e $n=(m+r)$, temos a verossimilhança profile (2.21) do modelo de captura-recaptura de Nayak.

Vamos analisar (2.41) para vários valores de $\mathrm{n}$ e ver o que acontece com o estimador de máxima verossimilhança de $\mathrm{N}, \hat{\mathrm{N}}_{\mathrm{P}}$.

Se $n=1$, temos que $w=1$ e

$$
L_{P}(N)=\frac{N !}{(N-1) ! N}=1, N \geq 1 .
$$

Então, $\hat{\mathrm{N}}_{\mathrm{P}}=$ qualquer número natural.

Se $n \geq 2$, temos:

i) para $\mathrm{w}=\mathrm{n}$

$$
L_{P}(N)=\frac{N !}{(N-n) ! N^{n}}=\frac{N(N-1) \ldots(N-(n-1))}{N^{n}}=\prod_{j=0}^{n-1}\left(1-\frac{j}{N}\right) \uparrow 1 \text { quando } N \rightarrow \infty
$$

e, portanto, $\hat{\mathrm{N}}_{\mathrm{P}}=\infty$. 
ii) $\operatorname{para} w=1$

$$
L_{P}(N)=\frac{N !}{(N-1) ! N^{n}}=\frac{1}{N^{n-1}}, N \geq 1
$$

Logo, $\mathrm{L}_{\mathrm{P}}(\mathrm{N}) \downarrow 0$ quando $\mathrm{N} \rightarrow \infty$ e portanto $\hat{\mathrm{N}}_{\mathrm{P}}=1$.

iii) para $1<\mathrm{w}<\mathrm{n}$, considere-se

$$
\begin{gathered}
\frac{L_{p}(N)}{L_{p}(N-1)}=\left(\frac{N}{N-w}\right)\left(1-\frac{1}{N}\right)^{n}, N \geq w+1, \\
g(w, n ; N)=\frac{L_{p}(N)}{L_{p}(N-1)}>1 \Leftrightarrow\left(1-\frac{1}{N}\right)^{n}>\frac{N-w}{N}=1-\frac{w}{N} \Leftrightarrow \\
g(w, n ; N)=\left(1-\frac{1}{N}\right)^{n}+\frac{w}{N}>1 .
\end{gathered}
$$

Se $g(w, n ; N)<1, \hat{N}_{P}=w . S e g(w, n ; N)>1$, então $\hat{N}_{P}=\max \{N \geq w+1$ : $\mathrm{g}(\mathrm{w}, \mathrm{n} ; \mathrm{N})>1\}$. De (2.42) segue que, dado $\mathrm{W}=\mathrm{w}, \hat{\mathrm{N}}_{\mathrm{P}}$ é uma função decrescente de $\mathrm{n}$ e $\hat{\mathrm{N}}_{\mathrm{P}}=\mathrm{k}$ se, e somente se, $\mathrm{h}_{\mathrm{w}, \mathrm{k}+1}<\mathrm{n}<\mathrm{h}_{\mathrm{w}, \mathrm{k}}$, onde

$$
\mathrm{h}_{\mathrm{w}, \mathrm{k}}=\frac{\log \left(1-\frac{\mathrm{w}}{\mathrm{k}}\right)}{\log \left(1-\frac{1}{\mathrm{k}}\right)}, \mathrm{k}>\mathrm{w},
$$


e $h_{w, w}=\infty$

Portanto, utilizando Processos de Poisson analisamos o problema de modelagem de uma maneira mais correta, pois não foi necessário observarmos os tempos de falhas como foi feito no modelo de captura-recaptura de Nayak. E os resultados obtidos (função de verossimilhança profile, e.m.v. de $\mathrm{N}$ e $\lambda$ ) são idênticos aos obtidos anteriormente.

\subsection{O Modelo de Goel e Okumoto}

Nos modelos vistos anteriormente, a correção de um erro não ocasiona outro. Este processo é muito questionado, principalmente em se tratando de software. Pensando nisto, Goel e Okumoto (1978) generalizaram o modelo de Jelinski e Moranda, incluindo uma probabilidade $\mathrm{p}$ de corrigir um erro quando este é encontrado.

A taxa de falha do modelo de Goel e Okumoto é dada por

$$
h\left(w_{i}\right)=\lambda[N-p(i-1)] .
$$

\subsubsection{Função de Verossimilhança}

A partir do momento em que observamos os dados, toda a informação sobre os parâmetros está contida na função de verossimilhança. A função de verossimilhança 
para os parâmetros $\mathrm{N}, \lambda$ e p do modelo de Goel e Okumoto é expressa por

$$
\mathrm{L}(\mathrm{N}, \lambda, \mathrm{p})=\lambda^{\mathrm{r}}\left(\prod_{\mathrm{i}=1}^{\mathrm{r}}[\mathrm{N}-\mathrm{p}(\mathrm{i}-1)]\right) \exp \left\{-\lambda\left[\sum_{\mathrm{i}=1}^{\mathrm{r}}[\mathrm{N}-\mathrm{p}(\mathrm{i}-1)] \mathrm{w}_{\mathrm{i}}\right]\right\}
$$

O logaritmo da função de verossimilhança (2.45) é dado por

$$
1(N, \lambda, p)=\sum_{i=1}^{r} \log [N-p(i-1)]+r \log (\lambda)-\lambda \sum_{i=1}^{r}[N-p(i-1)] w_{i}
$$

\subsubsection{Estimadores de Máxima Verossimilhança}

Os estimadores de máxima verossimilhança para os parâmetros do modelo de Goel e Okumoto, obtidos das equações de verossimilhança,

$$
\begin{aligned}
& \frac{\partial 1(N, \lambda, p)}{\partial N}=\sum_{i=1}^{r} \frac{1}{N-p(i-1)}-\lambda \sum_{i=1}^{r} w_{i}=0, \\
& \frac{\partial 1(N, \lambda, p)}{\partial p}=\sum_{i=1}^{r} \frac{(1-i)}{N-p(i-1)}-\lambda \sum_{i=1}^{r}(1-i) w_{i}=0, \\
& \frac{\partial 1(N, \lambda, p)}{\partial \lambda}=\frac{r}{\lambda}-\sum_{i=1}^{r}[N-p(i-1)] w_{i}=0,
\end{aligned}
$$


são dados pelas equações:

$$
\begin{aligned}
& \sum_{i=1}^{\mathrm{r}} \frac{1}{\hat{N}-\hat{p}(i-1)}-\hat{\lambda} \sum_{i=1}^{\mathrm{r}} \mathrm{w}_{\mathrm{i}}=0 \\
& \sum_{\mathrm{i}=1}^{\mathrm{r}} \frac{(1-\mathrm{i})}{\hat{N}-\hat{p}(\mathrm{i}-1)}-\hat{\lambda} \sum_{\mathrm{i}=1}^{\mathrm{r}}(1-\mathrm{i}) \mathrm{w}_{\mathrm{i}}=0
\end{aligned}
$$

e chamando $S_{\mathrm{r}}=\sum_{\mathrm{i}=1}^{\mathrm{r}} \mathrm{w}_{\mathrm{i}}$, teremos

$$
\hat{\lambda}=\frac{r}{\hat{N} S_{r}-\hat{p}\left(\sum_{i=1}^{r} i w_{i}-S_{r}\right)} .
$$

Devemos observar que para a obtenção dos e.m.v. de $\mathrm{N}$ e p, necessitamos do uso de um método iterativo, por exemplo, o método de Newton-Raphson.

\subsubsection{Inferências sobre os Parâmetros do Modelo}

Utilizando a aproximação Normal assintótica dos estimadores de máxima verossimilhança, consideramos

$$
(\hat{\mathrm{N}}, \hat{\lambda}, \hat{\mathrm{p}}) \stackrel{\mathrm{a}}{\sim} \mathrm{N}\left\{(\mathrm{N}, \lambda, \mathrm{p}) ; \mathrm{I}^{-1}(\hat{\mathrm{N}}, \hat{\lambda}, \hat{\mathrm{p}})\right\}
$$

onde $\mathrm{I}(\mathrm{N}, \lambda, \mathrm{p})$ é a matriz de informação de Fisher dada por 


$$
1(N, \lambda, p)=\left[\begin{array}{lll}
E\left\{\frac{-\partial^{2} 1(N, \lambda, p)}{\partial N^{2}}\right\} & E\left\{\frac{-\partial^{2} 1(N, \lambda, p)}{\partial N \partial \lambda}\right\} & \left.E\left\{\frac{-\partial^{2} 1(N, \lambda, p)}{\partial N \partial p}\right\}\right] \\
E\left\{\frac{-\partial^{2} 1(N, \lambda, p)}{\partial \lambda \partial N}\right\} & E\left\{\frac{-\partial^{2} 1(N, \lambda, p)}{\partial \lambda^{2}}\right\} & E\left\{\frac{-\partial^{2} 1(N, \lambda, p)}{\partial \lambda \partial p}\right\} \\
E\left\{\lambda^{2}\right\} & E\left\{\frac{-\partial^{2} 1(N, \lambda, p)}{\partial p \partial \lambda}\right\} & E\left\{\frac{-\partial^{2} 1(N, \lambda, p)}{\partial p^{2}}\right\}
\end{array}\right] .
$$

Assim, sabendo que $E\left(w_{i}\right)=\frac{1}{\lambda[N-p(i-1)]}$, obtemos

$$
1(N, \lambda \cdot p)=\left[\begin{array}{lll}
a_{11} & a_{12} & a_{13} \\
a_{12} & a_{22} & a_{23} \\
a_{13} & a_{23} & a_{33}
\end{array}\right]
$$

onde $a_{11}=\sum_{i=1}^{r}\left[\frac{1}{[N-p(i-1)]^{2}}\right], a_{12}=\frac{1}{\lambda} \sum_{i=1}^{r}\left[\frac{1}{[N-p(i-1)]}\right], a_{13}=\sum_{i=1}^{r}\left[\frac{(1-i)}{[N-p(i-1)]^{2}}\right]$,

$$
a_{22}=\frac{r}{\lambda^{2}}, a_{23}=\frac{1}{\lambda} \sum_{i=1}^{r}\left[\frac{(1-i)}{[N-p(i-1)]}\right] \text { e } a_{33}=\sum_{i=1}^{r}\left[\frac{(1-i)^{2}}{[N-p(i-1)]^{2}}\right]
$$

$\mathrm{I}^{-1}(\mathrm{~N}, \lambda, \mathrm{p})$, o inverso da matriz de informação de Fisher, dada em (2.55), representa a matriz de variância e covariância assintótica de $\hat{N}, \hat{\lambda}$ e $\hat{p}$. 
Considerando a distribuição Normal assintótica dos e.m.v., podemos construir intervalos de confiança para os parâmetros $N, \lambda$ e p. Considerando-se um nível de confiança $100(1-\alpha) \%$, os intervalos de confiança são dados por:

$$
\begin{aligned}
& \mathrm{P}\left[\hat{\mathrm{N}}-\mathrm{z}_{\alpha / 2} \sqrt{\mathrm{I}_{11}^{-1}(\mathrm{~N}, \lambda, \mathrm{p})} \leq \mathrm{N} \leq \hat{\mathrm{N}}+\mathrm{z}_{\alpha / 2} \sqrt{\mathrm{I}_{11}^{-1}(\mathrm{~N}, \lambda, \mathrm{p})}\right] \cong 1-\alpha, \\
& \mathrm{P}\left[\hat{\lambda}-\mathrm{z}_{\alpha / 2} \sqrt{\mathrm{I}_{22}^{-1}(\mathrm{~N}, \lambda, \mathrm{p})} \leq \lambda \leq \hat{\lambda}+\mathrm{z}_{\alpha / 2} \sqrt{\mathrm{I}_{22}^{-1}(\mathrm{~N}, \lambda, \mathrm{p})}\right] \cong 1-\alpha, \\
& \mathrm{P}\left[\hat{\mathrm{p}}-\mathrm{z}_{\alpha / 2} \sqrt{\mathrm{I}_{33}^{-1}(\mathrm{~N}, \lambda, \mathrm{p})} \leq \mathrm{p} \leq \hat{\mathrm{p}}+\mathrm{z}_{\alpha / 2} \sqrt{\mathrm{I}_{33}^{-1}(\mathrm{~N}, \lambda, \mathrm{p})}\right] \cong 1-\alpha,
\end{aligned}
$$

onde $\mathrm{I}_{\mathrm{i} i}^{-1}(\mathrm{~N}, \lambda, \mathrm{p}), \mathrm{i}=1,2,3$ representa o $\mathrm{i}$-ésimo elemento diagonal da matriz de variância e covariância assintótica de $\hat{\mathrm{N}}, \hat{\lambda}$ e $\hat{\mathrm{p}}, \mathrm{e} \mathrm{z}_{\alpha / 2}$ é um percentil da distribuição normal padronizada obtido de uma tabela estatística.

\subsection{O Modelo de Goel e Okumoto com Captura-Recaptura}

Nayak (1988), supondo que $\mathrm{p}=1$ no modelo de Goel e Okumoto (quando o software falha, o erro causador da falha é detectado e removido sem a inserção de outros erros), adaptou um contador $M_{i}$ para o i-ésimo erro corrigido, onde $M_{i}$ é o número de vezes com que a área $\mathrm{A}_{\mathrm{i}}$ é acessada. Este modelo, visto na seção 2.2, é conhecido como modelo de captura-recaptura dos erros corrigidos, onde o contador $\mathrm{M}_{\mathrm{i}}$ é utilizado para obter informação adicional sobre $\mathrm{N}$ e $\lambda$. 
Agora estenderemos a idéia de Nayak (1988) para $0 \leq \mathrm{p} \leq 1$. Seja $\mathrm{r}$, o número de erros descobertos no software após rodar $\tau$ unidades de tempo $(\mathrm{r} \geq 1)$, e dado $\mathrm{r}$ e $\mathrm{T}_{(1)} \leq \mathrm{T}_{(2)} \leq \ldots \leq \mathrm{T}_{(\mathrm{r})} \leq \tau$, os tempos de falhas sucessivas, seja $\mathrm{W}_{\mathrm{i}}=\mathrm{T}_{(\mathrm{i})}-\mathrm{T}_{(\mathrm{i}-1)}$; $\mathrm{i}=1, \ldots, \mathrm{r}+1$, onde $\mathrm{T}_{(0)}=0$ e $\mathrm{W}_{\mathrm{r}+1}=\mathrm{T}_{(\mathrm{r}+1)}-\mathrm{T}_{(\mathrm{r})}$ é censurado porque $\mathrm{W}_{\mathrm{r}+1}>\tau-\mathrm{T}_{(\mathrm{r})}$.

O modelo de Goel e Okumoto com captura-recaptura, baseado nos dados censurados $\mathrm{W}_{1}, \mathrm{~W}_{2}, \ldots, \mathrm{W}_{\mathrm{r}}, \mathrm{W}_{\mathrm{r}+1}$, pode ser formulado como:

$$
\begin{aligned}
& \mathrm{W}_{\mathrm{i}} \mid \mathrm{N}, \lambda \approx \operatorname{Exp}\{\lambda[\mathrm{N}-\mathrm{p}(\mathrm{i}-1)]\}, \mathrm{i}=1, \ldots, \mathrm{r}+1 \\
& \mathrm{M}_{\mathrm{i}} \approx \operatorname{Poisson}\left[\lambda\left(\tau-\mathrm{T}_{(\mathrm{i})}\right)\right], \mathrm{i}=1, \ldots \mathrm{r},
\end{aligned}
$$

onde a primeira parte corresponde ao modelo de correção imperfeita de Goel e Okumoto (1978), e a segunda parte corresponde aos contadores introduzidos por Nayak (1988).

\subsubsection{Função de Verossimilhança}

A função de verossimilhança é dada por

$$
\begin{gathered}
\mathrm{L}(\mathrm{N}, \lambda, \mathrm{p})=(\mathrm{r}+1) \prod_{\mathrm{i}=1}^{\mathrm{r}}\left\{\lambda[\mathrm{N}-\mathrm{p}(\mathrm{i}-1)] \exp \left\{-\lambda[\mathrm{N}-\mathrm{p}(\mathrm{i}-1)] \mathrm{w}_{\mathrm{i}}^{*}\right\}\right\} \times \\
\times \prod_{\mathrm{i}=1}^{\mathrm{r}}\left[\frac{\left[\lambda\left(\tau-\mathrm{t}_{(\mathrm{i})}\right)\right]^{\mathrm{m}_{\mathrm{i}}}}{\mathrm{m}_{\mathrm{i}} !} \exp \left\{-\lambda\left(\tau-\mathrm{t}_{(\mathrm{i})}\right)\right\}\right], \text { ou }
\end{gathered}
$$




$$
\begin{aligned}
& L(N, \lambda, p) \propto\left(\prod_{i=1}^{\mathrm{r}} \lambda[\mathrm{N}-\mathrm{p}(\mathrm{i}-1)]\right) \exp \left\{-\lambda \sum_{\mathrm{i}=1}^{\mathrm{r}+1}[\mathrm{~N}-\mathrm{p}(\mathrm{i}-1)] \mathrm{w}_{\mathrm{i}}^{*}\right\}\left(\prod_{\mathrm{i}=1}^{\mathrm{r}}\left[\lambda\left(\tau-\mathrm{t}_{(\mathrm{i})}\right)\right]^{\mathrm{m}_{\mathrm{i}}}\right) \times \\
& \times \exp \left\{-\lambda\left(\mathrm{r} \tau-\sum_{\mathrm{i}=1}^{\mathrm{r}} \mathrm{t}_{(\mathrm{i})}\right)\right\} \quad \operatorname{para} \mathrm{N} \geq \mathrm{r}, \lambda>0
\end{aligned}
$$

onde $\mathrm{w}_{\mathrm{i}}^{*}=\left\{\begin{array}{l}\mathrm{w}_{\mathrm{i}}, \mathrm{i}=1, \ldots, \mathrm{r} \\ \tau-\mathrm{t}_{(\mathrm{r})}, \mathrm{i}=\mathrm{r}+1\end{array}\right.$.

O teorema 2 será útil para desenvolvermos a função de verossimilhança.

TEOREMA 2: $\quad \sum_{\mathrm{i}=1}^{\mathrm{r}+1}[\mathrm{~N}-\mathrm{p}(\mathrm{i}-1)] \mathrm{w}_{\mathrm{i}}^{*}=\mathrm{p} \sum_{\mathrm{i}=1}^{\mathrm{r}} \mathrm{t}_{(\mathrm{i})}+(\mathrm{N}-\mathrm{r} \mathrm{p}) \tau$.

Prova:

$$
\begin{aligned}
& \sum_{i=1}^{r+1}[N-p(i-1)] w_{i}^{*}=N w_{1}+[N-p] w_{2}+\ldots+[N-p(r-1)] w_{r}+[N-r p] w_{r+1}^{*} \\
&= N t_{(1)}+[N-p]\left(t_{(2)}-t_{(1)}\right)+\ldots+[N-p(r-1)]\left(t_{(r)}-t_{(r-1)}\right)+[N-r p]\left(\tau-t_{(r)}\right) \\
&= N t_{(1)}+[N-p] t_{(2)}-[N-p] t_{(1)}+\ldots+[N-r p+p] t_{(r)}-[N-r p+p] t_{(r-1)}+ \\
&+[N-r p]\left(\tau-t_{(r)}\right) \\
&= t_{(1)}[N-N+p]+t_{(2)}[N-p+N-2 p]+\ldots+t_{(r-1)}[N-r p+2 p-N+r p-p]+ \\
&+t_{(r)}[N-r p+p-N+r p]+[N-r p] \tau
\end{aligned}
$$


$=p t_{(1)}+p t_{(2)}+\ldots+p t_{(r-1)}+p t_{(r)}+[N-r p] \tau=p \sum_{i=1}^{r} t_{(i)}+(N-r p) \tau$

Utilizando-se o teorema 2, o logaritmo da função de verossimilhança é dado por

$$
\begin{gathered}
1(\mathrm{~N}, \lambda, \mathrm{p}) \propto \mathrm{r} \log (\lambda)+\sum_{\mathrm{i}=1}^{\mathrm{r}} \log [\mathrm{N}-\mathrm{p}(\mathrm{i}-1)]-\lambda\left\{\mathrm{N} \tau+(1-\mathrm{p})\left[\mathrm{r} \tau-\sum_{\mathrm{i}=1}^{\mathrm{r}} \mathrm{t}_{(\mathrm{i})}\right]\right\}+ \\
+\log \left(\prod_{\mathrm{i}=1}^{\mathrm{r}}\left[\lambda\left(\tau-\mathrm{t}_{(\mathrm{i})}\right)\right]^{\mathrm{m}_{\mathrm{i}}}\right)
\end{gathered}
$$

\subsubsection{Estimadores de Máxima Verossimilhança}

Fazendo as derivadas parciais de (2.59) e igualando a zero, teremos:

$$
\begin{aligned}
& \frac{\partial 1(N, \lambda, p)}{\partial N}=\sum_{i=1}^{r} \frac{1}{[N-p(i-1)]}-\lambda \tau=0, \\
& \frac{\partial 1(N, \lambda, p)}{\partial \lambda}=\frac{r+m}{\lambda}-N \tau-(1-p)\left[r \tau-\sum_{i=1}^{r} t_{(i)}\right]=0, \\
& \frac{\partial 1(N, \lambda, p)}{\partial p}=\sum_{i=1}^{r} \frac{(i-1)}{[N-p(i-1)]}+\lambda\left[r \tau-\sum_{i=1}^{r} t_{(i)}\right]=0 .
\end{aligned}
$$


Para obtermos os e.m.v. de $\mathrm{N}$ e p, devemos utilizar um método iterativo, como por exemplo, o de Newton-Raphson (ver apêndice 1).

Então, dado $\hat{\mathrm{N}}$ e $\hat{\mathrm{p}}$, o estimador de máxima verossimilhança de $\lambda$ é dado por

$$
\hat{\lambda}=\frac{\mathrm{r}+\mathrm{m}}{\hat{\mathrm{N}} \tau+(1-\hat{\mathrm{p}})\left[\mathrm{r} \tau-\sum_{\mathrm{i}=1}^{\mathrm{r}} \mathrm{t}_{(\mathrm{i})}\right]} .
$$

Observe-se que para $\mathrm{p}=1$, nosso estimador de $\lambda$ coincide com o estimador obtido em (2.19). Naquela seção 2.2 vimos que, quando $p=1$, os tempos de falhas não davam informação extra sobre os parâmetros do modelo, e, portanto, eles estavam sendo observados desnecessariamente. Com $0 \leq \mathrm{p}<1$, os tempos de falhas são relevantes, ou seja, eles nos dão alguma informação sobre o parâmetro do modelo.

Inferências sobre os parâmetros do modelo, como por exemplo, intervalos de confiança, podem ser obtidas utilizando-se a aproximação Normal assintótica dos estimadores de máxima verossimilhança, baseada na matriz de informação de Fisher ou informação observada.

\subsubsection{Função de Verossimilhança Profile para N (p conhecido)}

Para obter o e.m.v. de N (considerando p conhecido), vamos utilizar a função de verossimilhança profile $L_{\mathrm{P}}(\mathrm{N})$, que é dada por 


$$
L_{P}(N)=\frac{\prod_{i=1}^{\mathrm{r}}[N-p(i-1)]}{\left\{N \tau+(1-p)\left[r \tau-\sum_{i=1}^{r} t_{(i)}\right]\right\}^{\mathrm{r}+\mathrm{m}}}, N \geq \mathrm{r} .
$$

Usando o mesmo procedimento da seção 2.2 .4 , nós obtemos da função de verossimilhança profile, o e.m.v. de $\mathrm{N}, \hat{\mathrm{N}}_{\mathrm{p}}$, que é obtido da seguinte forma:

Seja

$$
g(N)=\frac{L_{p}(N)}{L_{p}(N-1)}=\left[1-\frac{1}{N+(1-p) v}\right]^{\mathrm{r}+\mathrm{m}}+1-\prod_{i=1}^{\mathrm{r}}\left[1-\frac{1}{N-p(i-1)}\right]
$$

onde $\mathrm{N} \geq \mathrm{r}+1$ e $\mathrm{v}=\frac{\mathrm{r} \tau-\sum_{\mathrm{i}=1}^{\mathrm{r}} \mathrm{t}_{(\mathrm{i})}}{\tau}$

Se $g(r+1)<1$, então $\hat{N}_{p}=r$. Se $g(r+1)>1$, então $\hat{N}_{p}=k$ se, e somente se, $\mathrm{h}_{\mathrm{r}, \mathrm{k}+1}^{*}<\mathrm{m}<\mathrm{h}_{\mathrm{r}, \mathrm{k}}^{*}$, onde

$$
h_{r, k}^{*}=\frac{\sum_{i=1}^{r} \log \left(1-\frac{1}{k-p(i-1)}\right)}{\log \left\{1-\frac{1}{k+(1-p) v}\right\}}-r .
$$

Se $\mathrm{p}=1, \mathrm{~h}_{\mathrm{r}, \mathrm{k}}^{*}$ coincide com $\mathrm{h}_{\mathrm{r}, \mathrm{k}}$ obtido em (2.23). 


\subsubsection{Estimativa da Média e Variância de $\hat{\mathrm{N}}_{\mathrm{p}}$}

Estamos interessados em obter uma estimativa pontual, como a média e a variância de $\hat{\mathrm{N}}_{\mathrm{p}}$. Seber (1982) obteve uma estimativa assintótica da média e variância de $\hat{\mathrm{N}}$. Procedendo da mesma maneira, obteremos as estimativas pontuais.

Seja

$$
\frac{L_{p}(N)}{L_{p}(N-1)}=1 \Rightarrow\left[1-\frac{1}{N+(1-p) v}\right]^{\mathrm{r}+\mathrm{m}} \prod_{i=1}^{\mathrm{r}}\left[\frac{[\mathrm{N}-\mathrm{p}(\mathrm{i}-1)]}{[\mathrm{N}-1-\mathrm{p}(\mathrm{i}-1)]}\right]=1
$$

Desenvolvendo o produtório em (2.67) e supondo N suficientemente grande, teremos:

$$
\begin{aligned}
& {\left[1-\frac{1}{N+(1-p) v}\right]^{\mathrm{r}+\mathrm{m}}\left[\frac{\mathrm{N}}{\mathrm{N}-[1+(\mathrm{r}-1) \mathrm{p}]}\right]=1 } \\
\Rightarrow & \prod_{\mathrm{i}=1}^{\mathrm{r}+\mathrm{m}}\left[\frac{\mathrm{N}-(\mathrm{p}-1) \mathrm{v}-1}{\mathrm{~N}-(\mathrm{p}-1) \mathrm{v}}\right]=\left[1-\frac{1+(\mathrm{r}-1) \mathrm{p}}{\mathrm{N}}\right] .
\end{aligned}
$$

Assim, a média assintótica de $\hat{\mathrm{N}}_{\mathrm{p}}$ é estimada por

$$
E\left[\hat{N}_{p}\right]=N+b
$$

onde b, o vício, é estimado por 


$$
\hat{b}=\frac{\left[\frac{1}{\hat{N}_{p}}+\sum_{i=1}^{r+m}\left(\frac{1}{\hat{N}_{p}-(p-1) v-1}-\frac{1}{\hat{N}_{p}-(p-1) v}\right)\right]^{2}-\left[\frac{1}{\hat{N}_{p}^{2}}+\sum_{i=1}^{r+m} \frac{1}{\left[\hat{N}_{p}-(p-1) v-1\right]^{2}}-\frac{1}{\left[\hat{N}_{p}-(p-1) v\right]^{2}}\right]}{2\left[\frac{1}{\hat{N}_{p}-[1+(r-1) p}-\frac{1}{\hat{N}_{p}}-\sum_{i=1}^{r+m}\left[\frac{1}{\left[\hat{N}_{p}-(p-1) v-1\right]\left[\hat{N}_{p}-(p-1) v\right]}\right]\right]^{2}}
$$

e a variância assintótica de $\hat{\mathrm{N}}_{\mathrm{p}}$ é estimada por (ver Seber, 1982)

$$
\hat{\mathrm{V}}\left[\hat{\mathrm{N}}_{\mathrm{p}}\right]=\left[\frac{1}{\hat{\mathrm{N}}_{\mathrm{p}}-[1+(\mathrm{r}-1) \mathrm{p}}-\frac{1}{\hat{\mathrm{N}}_{\mathrm{p}}}-\sum_{\mathrm{i}=1}^{\mathrm{r}+\mathrm{m}}\left(\frac{1}{\left[\hat{\mathrm{N}}_{\mathrm{p}}-(\mathrm{p}-1) \mathrm{v}-1\right]\left[\hat{\mathrm{N}}_{\mathrm{p}}-(\mathrm{p}-1) \mathrm{v}\right]}\right)\right]^{-1},
$$

onde $\mathrm{v}=\frac{\mathrm{r} \tau-\sum_{\mathrm{i}=1}^{\mathrm{r}} \mathrm{t}_{(\mathrm{i})}}{\tau}$

Considerando a distribuição Normal assintótica para amostras grandes, ou seja,

$$
\hat{\mathrm{N}} \stackrel{\mathrm{a}}{\sim} N\left\{[\mathrm{~N}+\mathrm{b}] ; \hat{\mathrm{V}}\left[\hat{\mathrm{N}}_{\mathrm{p}}\right]\right\}
$$

podemos construir um intervalo de confiança para o parâmetro N. 


\section{CAPÍTULO 3}

\section{ANÁLISE BAYESIANA}

\subsection{Introdução}

Os métodos Bayesianos constituem um conjunto de técnicas alternativas à análise clássica, que podem facilitar a interpretação dos resultados e que permitem a incorporação de informações sobre os parâmetros do modelo antes de observarmos os dados.

Esse conhecimento prévio é expresso através de uma densidade de probabilidade, conhecida como densidade a priori, a qual é atribuída aos parâmetros, que, sob esse ponto de vista, são considerados variáveis aleatórias. Quando não temos nenhuma informação a respeito dos parâmetros, devemos formular uma priori tal que toda informação venha exclusivamente dos dados, ou seja, devemos considerar uma priori não-informativa.

O Teorema de Bayes proporciona a obtenção de uma densidade a posteriori, a qual combina a informação vinda dos dados, através da função de verossimilhança, com a informação prévia, representada na densidade a priori (ver por exemplo, Box e Tiao, 1973). 


\subsection{Fórmula de Bayes}

Sejam $\mathrm{X}$ e $\theta$ variáveis aleatórias com densidades conjuntas e condicionais dadas por $f(x, \theta), f(x \mid \theta)$ e $p(\theta \mid x)$, respectivamente, e seja $p(\theta)$ a densidade marginal de $\theta$. A densidade condicional de $\theta$, dado $\mathrm{x}$, é dada por

$$
p(\theta \mid x)=\frac{f(x, \theta)}{f(x)}=\frac{f(x \mid \theta) p(\theta)}{\int_{\theta \in \Theta} f(x \mid \theta) p(\theta) d \theta}
$$

onde $\Theta$ é o espaço paramétrico.

Como o termo à esquerda da igualdade é uma densidade para $\theta$, a observação $\mathrm{x}$ é apenas uma constante bem como $\mathrm{f}(\mathrm{x})$. Além disso, a posteriori será daqui em diante denotada por $\pi(\theta)$, ficando subentendido o valor da observação na qual ela foi condicionada. Logo, o teorema de Bayes pode ser escrito em uma forma mais resumida como

$$
\pi(\theta) \propto 1(\theta) p(\theta)
$$

onde $1(\theta)=f(x \mid \theta)$ é a função de verossimilhança de $\theta$.

\subsection{Densidades a Priori e a Posteriori para os Parâmetros}

Antes de analisar estatisticamente os dados, devemos incorporar o nosso 
conhecimento prévio a respeito dos parâmetros do modelo. Uma maneira conveniente de se fazer isto é formular uma densidade de probabilidade para os parâmetros do modelo, identificada como densidade a priori.

Quando não temos nenhuma informação a respeito dos parâmetros, devemos formular uma priori tal que toda informação acerca dos parâmetros seja exclusivamente dos dados, ou seja, devemos considerar uma priori nãoinformativa.

Uma vez definidos o modelo para os dados e a distribuição a priori, devemos combinar a informação prévia sobre os parâmetros com a informação contida nos dados, obtendo assim uma distribuição a posteriori para os parâmetros do modelo.

Uma preocupação que devemos ter, quando escolhemos priori nãoinformativas, é quanto à existência da distribuição a posteriori. Com priori informativas, a distribuição a posteriori sempre existirá.

Uma vez obtida a distribuição a posteriori, pode-se procurar sumarizar a informação nela contida através de alguns elementos. Em particular, podem ser calculadas medidas de locação para fornecer uma idéia de possíveis valores centrais e de dispersão para dar uma idéia da variabilidade associada à situação descrita pela posteriori. As principais medidas de posição são a média, a moda e a mediana e as principais medidas de dispersão são a variância e o desvio-padrão. A média a posteriori é o valor esperado de $\theta$ sob $\pi$, a moda é o valor mais provável de $\pi$ e a mediana divide o espaço paramétrico em duas partes com igual probabilidade. A variância no caso multivariado é dada pela matriz de variâncias-covariâncias e o 
desvio-padrão, nesse caso, pode ser visto como a raiz quadrada dos elementos na diagonal da variância.

Exceto a mediana, todas as medidas descritas acima podem ser calculadas indistintamente para distribuições conjuntas, marginais ou condicionais mas a mediana só faz sentido para distribuições univariadas.

\subsection{Algoritmo Gibbs Sampling}

Uma das limitações dos métodos Bayesianos é a resolução de integrais para a obtenção das distribuições marginais e conseqüentemente dos resumos a posteriori. Quando as integrais não apresentam soluções analíticas explícitas, é necessário o uso de métodos de aproximação de integrais, como, por exemplo, o de Laplace (ver por exemplo, Tierney e Kadane, 1986).

Uma outra alternativa para contornar esse problema é utilizar técnicas de simulação, como o método de Gibbs Sampling (ver por exemplo, Casella e George, 1992). Este método gera, indiretamente, uma amostra aleatória de uma distribuição marginal sem ter que calcular sua densidade (devido a problemas de resoluções de integrais).

O método de Gibbs é essencialmente um esquema iterativo de amostragem de uma cadeia de Markov cujo núcleo de transição é formado pelas distribuições condicionais. Para descrever o algoritmo, suponha-se que a distribuição de interesse é $\pi(\theta)$, onde $\theta=\left(\theta_{1}, \theta_{2}, \ldots, \theta_{\mathrm{p}}\right)$. Considere-se ainda que as densidades condicionais a 
posteriori $\pi_{\mathrm{i}}\left(\theta_{\mathrm{i}}\right)=\pi\left(\theta_{\mathrm{i}} \mid \theta_{\mathrm{r}}\right), \mathrm{i}=1, \ldots, \mathrm{p}$ estão disponíveis.

O problema que se coloca é a dificuldade de geração de uma amostra de $\pi$, supondo-se que essa geração direta de $\pi$ é extremamente complicada, mas ela é possível de ser realizada. O algoritmo de Gibbs, então, fornece uma forma alternativa de geração baseada em sucessivas gerações das distribuições condicionais. Ele é descrito da seguinte forma:

i) inicie o contador de iterações da cadeia $\mathrm{j}=1$ e arbitre valores iniciais $\theta^{(0)}=\left(\theta_{1}^{(0)}, \ldots, \theta_{\mathrm{p}}^{(0)}\right)$,

ii) obtenha um novo valor $\theta^{(\mathrm{j})}=\left(\theta_{1}{ }^{(\mathrm{j})}, \ldots, \theta_{\mathrm{p}}{ }^{(\mathrm{j})}\right)$ a partir de $\theta^{(\mathrm{j}-1)}$ através de sucessivas gerações de valores:

$$
\begin{aligned}
\theta_{1}^{(j)} & \sim \pi\left(\theta_{1} \mid \theta_{2}{ }^{(j-1)}, \ldots, \theta_{\mathrm{p}}^{(\mathrm{j}-1)}\right) \\
\theta_{2}^{\left({ }^{(j)}\right)} & \sim \pi\left(\theta_{2} \mid \theta_{1}{ }^{(\mathrm{j})}, \theta_{3}{ }^{(\mathrm{j}-1)}, \ldots, \theta_{\mathrm{p}}^{(\mathrm{j}-1)}\right) \\
& : \\
& : \\
\theta_{\mathrm{p}}^{(\mathrm{j})} & \sim \pi\left(\theta_{\mathrm{p}} \mid \theta_{1}{ }^{(\mathrm{j})}, \ldots, \theta_{\mathrm{p}-1}{ }^{(\mathrm{j}-1)}\right)
\end{aligned}
$$

iii) mude o contador $\mathrm{j}$ para $(\mathrm{j}+1)$ e retorne a (ii) até a convergência.

À medida que o número de iterações aumenta, a cadeia se aproxima de sua condição de equilíbrio. Assim, assume-se que a convergência é atingida em uma iteração cuja distribuição esteja arbitrariamente próxima da distribuição de equilíbrio $\pi$. 


\subsubsection{Verificação da Convergência}

Uma das propostas iniciais de verificação de convergência foi feita informalmente por Gelfand e Smith (1990).

Eles sugeriram técnicas gráficas para a verificação da convergência. Após um número suficientemente grande $\mathrm{N}$ de iterações em $m$ cadeias paralelas, forma-se uma amostra de $\theta$ e pode-se construir um histograma de qualquer uma de suas componentes. $\mathrm{O}$ mesmo procedimento pode ser repetido até $\mathrm{N}+\mathrm{k}$ iterações. Se não houver diferença perceptível entre os gráficos obtidos após $\mathrm{N}$ e $(\mathrm{N}+\mathrm{k})$ iterações, então conclui-se pela convergência das cadeias.

Ainda dentro da linha de verificação gráfica, é possível observar a trajetória de uma única cadeia ao longo das iterações. Se o gráfico, após um período inicial, apresenta repetidamente o mesmo comportamento qualitativo e quantitativo, então pode-se concluir pela convergência da cadeia.

Uma outra forma simples de verificar a convergência é a utilização de várias cadeias em paralelo começando de diferentes pontos. Com isso, evita-se que as cadeias se concentrem em regiões em torno de uma moda local, no caso de multimodalidade da posteriori.

Gelman e Rubin (1992) propuseram um método formal para a deteç̧ão de convergência além da verificação visual. Esse método é baseado em técnicas de análise de variância e sugere a convergência da cadeia apenas quando a variância entre as cadeias for bem menor que a variância dentro de cada cadeia ou, 
equivalentemente, quando histogramas das cadeias misturadas são similares aos de cada uma delas isoladas.

Considerando $m$ cadeias paralelas e uma função real $\mathrm{t}(\theta)$, tem-se $m$ trajetórias $\left\{\mathrm{t}_{\mathrm{i}}^{(1)}, \mathrm{t}_{\mathrm{i}}^{(2)}, \ldots, \mathrm{t}_{\mathrm{i}}^{(\mathrm{n})}, \mathrm{i}=1, \ldots, m\right.$ para $\mathrm{t}$. Portanto, podem ser obtidas a variância entre as cadeias (E) e a variância dentro das cadeias (D), dadas por:

$$
E=\frac{n}{m-1} \sum_{i=1}^{m}\left(\bar{t}_{i}-\bar{t}\right)^{2}
$$

e

$$
\mathrm{D}=\frac{1}{\mathrm{~m}(\mathrm{n}-1)} \sum_{\mathrm{i}=1}^{\mathrm{m}} \sum_{\mathrm{j}=1}^{\mathrm{n}}\left(\mathrm{t}_{\mathrm{i}}^{(\mathrm{j})}-\overline{\mathrm{t}}_{\mathrm{i}}\right)^{2}
$$

onde $\overline{\mathrm{t}}_{\mathrm{i}}$ é a média das observações da cadeia $i$ e $\overline{\mathrm{t}}$ é a média dessas médias, $\mathrm{i}=1, . ., m$. Sob convergência, todos os $m n$ valores são gerados da posteriori e a variância de $t$ pode ser estimada de forma não-viciada por

$$
\hat{\mathrm{V}}[\mathrm{t}(\theta)]=\left(1-\frac{1}{\mathrm{n}}\right) \mathrm{D}+\left(\frac{1}{\mathrm{n}}\right) \mathrm{E}
$$

Um indicador de convergência é dado pela redução potencial estimada de escala $\mathrm{R}=\sqrt{\hat{\mathrm{V}}[\mathrm{t}(\theta)] / \mathrm{D}}$. À medida que $\mathrm{n}$ cresce, $\mathrm{R}$ convergirá para 1 . Logo, $\mathrm{R}$ pode ser usado como indicador de convergência pela avaliação de sua proximidade a 1 . Quando a convergência está assegurada, utiliza-se a última metade dos valores gerados para obter inferências sobre a distribuição desejada. 


\subsection{Algoritmo de Metropolis-Hastings}

Considere-se uma distribuição $\pi$, da qual se deseja gerar uma amostra através de uma cadeia de Markov, visto que a geração não-iterativa de $\pi$ é muito complicada. Nesse caso, deve-se construir um núcleo de transição $p(\theta, \phi)$, de forma que $\pi$ seja a distribuição de equilíbrio da cadeia. Uma forma simples de fazer isso é através de cadeias onde o núcleo p satisfaça a condição de reversibilidade dada por

$$
\pi(\theta) \mathrm{p}(\theta, \phi)=\pi(\phi) \mathrm{p}(\phi, \theta), \forall(\theta, \phi)
$$

A equação (3.3) é também conhecida como equação de equilíbrio detalhado (Green, 1995). Embora não seja necessária, ela é condição suficiente para que $\pi$ seja a distribuição de equilíbrio da cadeia.

O núcleo $\mathrm{p}(\theta, \phi)$ é constituído de dois elementos: um núcleo de transição arbitrário $\mathrm{q}(\theta, \phi)$ e uma probabilidade $\alpha(\theta, \phi)$. de forma que

$$
\mathrm{p}(\theta, \phi)=\mathrm{q}(\theta, \phi) \alpha(\theta, \phi), \theta \neq \phi
$$

Portanto, o núcleo de transição define uma densidade $\mathrm{p}(\theta, \cdot)$ para todos os valores diferentes de $\theta$. Conseqüentemente, resta uma probabilidade positiva de a cadeia ficar em $\theta$ dada por

$$
p(\theta, \theta)=1-\int q(\theta, \phi) \alpha(\theta, \phi) d \phi .
$$


Essas duas formas podem ser agrupadas na expressão geral

$$
p(\theta, A)=\int_{A} q(\theta, \phi) \alpha(\theta, \phi) d \phi+I(\theta \in A)\left[1-\int q(\theta, \phi) \alpha(\theta, \phi) d \phi\right]
$$

Logo, o núcleo de transição define uma distribuição mista para o novo estado $\phi$ da cadeia. Para $\phi \neq \theta$, essa distribuição tem densidade e para $\phi=\theta$, essa distribuição atribui uma probabilidade positiva.

A expressão mais citada para a probabilidade de aceitação é

$$
\alpha(\theta, \phi)=\min \left\{1, \frac{\pi(\phi) \mathrm{q}(\phi, \theta)}{\pi(\theta) \mathrm{q}(\theta, \phi)}\right\}
$$

onde a razão que aparece na expressão é chamada por Hastings (1970) de razão de teste.

A simulação de uma amostra de $\pi$, usando-se a cadeia de Markov definida pela transição (3.4), pode ser esquematizada da seguinte forma:

i) inicie o contador de iterações da cadeia $j=1$ e arbitre o valor inicial $\theta^{(0)}$;

ii) mova a cadeia para um novo valor $\phi$ gerado da densidade $\mathrm{q}\left(\theta^{(\mathrm{j}-1)}, \cdot\right)$;

iii) calcule a probabilidade de aceitação do movimento $\alpha\left(\theta^{(\mathrm{j}-1)}, \phi\right)$ dada por (3.5). Se o movimento for aceito, $\theta^{(j)}=\phi$. Se não for aceito, $\theta^{(j)}=\theta^{(j-1)}$ e a cadeia 
não se move;

iv) mude o contador de $\mathrm{j}$ para $(\mathrm{j}+1)$ e retorne a (ii) até a convergência.

A etapa (iii) é realizada após a geração de uma quantidade uniforme $u$ independente de todas as outras variáveis. Se $u \leq \alpha$, o movimento é aceito e se $u>\alpha$, o movimento não é permitido. O núcleo de transição q define apenas uma proposta de movimento, que pode ou não ser confirmado por $\alpha$.

O sucesso do método depende de taxas de aceitação não muito baixas e de propostas fáceis de simular. O método substitui uma geração difícil de $\pi$ por várias gerações fáceis de q. Isso só faz sentido se for fácil gerar de q.

As técnicas formais e informais de convergência descritas na seção 3.4.1 também podem ser utilizadas aqui.

\subsubsection{Metropolis em Gibbs}

No caso limite, em que se sabe como gerar valores de todas as distribuições condicionais, obtém-se o amostrador de Gibbs descrito na seção 3.4. Geralmente em modelos complexos, é possível estabelecer conjugação condicional para algumas mas não para todas as componentes do modelo.

Nesse caso, sugere-se que a geração das componentes $\theta_{i}$, para as quais não se sabe gerar diretamente de $\pi_{i}$, seja feita através de uma (sub)cadeia de Metropolis- 
Hastings dentro do ciclo do amostrador de Gibbs. Esse processo se daria ao longo de $\mathrm{T}$ iterações, culminando com a geração de $\theta_{\mathrm{i}}$ dentro de uma iteração do amostrador de Gibbs. O valor de $\mathrm{T}$ seria determinado de forma a garantir a convergência da subcadeia para a sua distribuição de equilíbrio $\pi_{\mathrm{i}}$. Este esquema é denominado de esquema amostral de Metropolis em Gibbs.

\subsection{Análise Bayesiana do Modelo de Jelinski e Moranda}

Nesta seção, vamos obter inferências sobre os parâmetros do modelo através de uma análise Bayesiana para o modelo de Jelinski e Moranda, considerando a densidade a priori informativa e explorando os métodos de Gibbs Sampling e Metropolis-Hastings.

A função de verossimilhança para os parâmetros $\mathrm{N}$ e $\lambda$ do modelo de Jelinski e Moranda é dada por

$$
\mathrm{L}(\mathrm{N}, \lambda)=\lambda^{\mathrm{r}}\left(\prod_{\mathrm{i}=1}^{\mathrm{r}}(\mathrm{N}-\mathrm{i}+1)\right) \exp \left\{-\lambda\left[\sum_{\mathrm{i}=1}^{\mathrm{r}} \mathrm{t}_{(\mathrm{i})}+(\mathrm{N}-\mathrm{r}) \mathrm{t}_{(\mathrm{r})}\right]\right\}
$$

Dado o espaço paramétrico dos parâmetros, assumiremos uma densidade a priori Gama para $\lambda$ e uma densidade a priori Poisson para $N$, dadas por:

$$
\lambda \sim \Gamma[\mathrm{a}, \mathrm{b}] \quad \text { e } \quad \mathrm{N} \sim \mathrm{P}(\gamma) .
$$


Supondo-se independência a priori e $a, b$ e $\gamma$ conhecidos, a densidade a priori conjunta para $\mathrm{N}$ e $\lambda$ é dada por

$$
p(N, \lambda) \propto \frac{e^{-\gamma} \gamma^{N}}{N !} \lambda^{a-1} e^{-b \lambda}
$$

onde $\mathrm{N}=\mathrm{r}, \mathrm{r}+1, \ldots$ e $\lambda>0$.

A densidade a posteriori conjunta para $\mathrm{N}$ e $\lambda$ é dada por

$$
\pi(\mathrm{N}, \lambda) \propto \mathrm{p}(\mathrm{N}, \lambda) \mathrm{L}(\mathrm{N}, \lambda)
$$

onde $L(N, \lambda)$ é a função de verossimilhança (3.6) e p(N, $\lambda)$ representa a densidade a priori (3.7).

A densidade a posteriori conjunta para os parâmetros do modelo de Jelinski e Moranda é dada por

$$
\pi(\mathrm{N}, \lambda) \propto \frac{\gamma^{\mathrm{N}} \lambda^{\mathrm{ra-1}-1}}{\mathrm{~N} !}\left(\prod_{\mathrm{i}=1}^{\mathrm{r}}(\mathrm{N}-\mathrm{i}+1)\right) \exp \left\{-\lambda\left[\mathrm{b}+\sum_{\mathrm{i}=1}^{\mathrm{r}} \mathrm{t}_{(\mathrm{i})}+(\mathrm{N}-\mathrm{r}) \mathrm{t}_{(\mathrm{r})}\right]\right\},
$$

onde $\mathrm{N}=\mathrm{r}, \mathrm{r}+1, \ldots$ e $\lambda>0$.

Em inferência Bayesiana, os resumos (média, moda, variância, etc.) são tipicamente baseados nas densidades a posteriori marginais dos parâmetros envolvidos. Para obtermos uma amostra da densidade a posteriori marginal de $\mathrm{Ne}$ 
de $\lambda$, usamos o método de Gibbs Sampling, que se baseia em sucessivas gerações das distribuições condicionais a posteriori $(N \mid \lambda)$ e $(\lambda \mid N)$.

As densidades a posteriori condicionais de $\mathrm{N}$ e $\lambda$ para o algoritmo de Gibbs podem ser obtidas, respectivamente, por:

$$
\pi(\mathrm{N} \mid \lambda)=\frac{\pi(\mathrm{N}, \lambda)}{\pi(\lambda)} \quad \text { e } \quad \pi(\lambda \mid \mathrm{N})=\frac{\pi(\mathrm{N}, \lambda)}{\pi(\mathrm{N})}
$$

onde $\pi(\mathrm{N})$ e $\pi(\lambda)$ são as densidades a posteriori marginais de $\mathrm{Ne} \lambda$. Assim,

$$
\begin{aligned}
& \pi(\mathrm{N} \mid \lambda) \propto \frac{\gamma^{\mathrm{N}}}{\mathrm{N} !}\left(\prod_{\mathrm{i}=1}^{\mathrm{r}}(\mathrm{N}-\mathrm{i}+1)\right) \exp \left\{-\lambda(\mathrm{N}-\mathrm{r}) \mathrm{t}_{(\mathrm{r})}\right\} \\
& \lambda \mid \mathrm{N} \sim \Gamma\left[\mathrm{r}+\mathrm{a}, \mathrm{b}+\sum_{\mathrm{i}=1}^{\mathrm{r}} \mathrm{t}_{(\mathrm{i})}+(\mathrm{N}-\mathrm{r}) \mathrm{t}_{(\mathrm{r})}\right] .
\end{aligned}
$$

Podemos reescrever a densidade a posteriori condicional de $\mathrm{N}$ como

$$
\pi(\mathrm{N} \mid \lambda) \propto \frac{\gamma^{\mathrm{N}}}{(\mathrm{N}-\mathrm{r}) !} \exp \left\{-\lambda(\mathrm{N}-\mathrm{r}) \mathrm{t}_{(\mathrm{r})}\right\}
$$

pois $\frac{\prod_{i=1}^{r}(N-i+1)}{N !}=\frac{1}{(N-r) !}$. 
Definindo a transformação $\mathrm{N}^{\prime}=\mathrm{N}-\mathrm{r}$, temos que

$$
\pi(\mathrm{N} \mid \lambda) \propto \frac{\left[\left(\gamma \exp \left(-\lambda \mathrm{t}_{(\mathrm{r})}\right)\right]^{\mathrm{N}^{\prime}} \exp \left\{-\gamma \lambda \mathrm{t}_{(\mathrm{r})}\right\}\right.}{\mathrm{N}^{\prime} !},
$$

onde $N^{\prime}=0,1,2, \ldots$ é uma distribuição de Poisson com média $\left[\gamma \exp \left(-\lambda t_{(r)}\right)\right]$.

Assim, as densidades condicionais requeridas para o algoritmo de Gibbs Sampling são dadas por:

$$
\begin{aligned}
& \lambda \mid \mathrm{N}^{\prime} \sim \Gamma\left[\mathrm{r}+\mathrm{a}, \mathrm{b}+\sum_{\mathrm{i}=1}^{\mathrm{r}} \mathrm{t}_{(\mathrm{i})}+\mathrm{N}^{\prime} \mathrm{t}_{(\mathrm{r})}\right], \\
& \mathrm{N}^{\prime} \mid \lambda \sim \mathrm{P}\left[\gamma \exp \left(-\lambda \mathrm{t}_{(\mathrm{r})}\right)\right] .
\end{aligned}
$$

\subsection{Análise Bayesiana do Modelo de Captura-Recaptura de Nayak}

A função de verossimilhança para o modelo de captura-recaptura é expressa por

$$
\mathrm{L}(\mathrm{N}, \lambda) \propto \lambda^{(\mathrm{r}+\mathrm{m})} \mathrm{e}^{-\mathrm{N} \lambda \tau} \prod_{\mathrm{i}=1}^{\mathrm{r}}(\mathrm{N}-\mathrm{i}+1)
$$

Para esse modelo, iremos considerar densidades a priori não-informativas para $\mathrm{N}$ e $\lambda$, dadas por: 


$$
\mathrm{p}(\mathrm{N}) \propto \frac{1}{\mathrm{~N}}
$$

e

$$
p(\lambda) \propto \frac{1}{\lambda}
$$

Supondo-se independência a priori, a densidade a priori conjunta para $\mathrm{N}$ e $\lambda$ é dada por

$$
\mathrm{p}(\mathrm{N}, \lambda) \propto \frac{1}{\mathrm{~N} \lambda}
$$

A densidade a posteriori conjunta é expressa por

$$
\pi(\mathrm{N}, \lambda) \propto \lambda^{(\mathrm{r}+\mathrm{m}-1)} \mathrm{e}^{-\mathrm{N} \lambda \tau} \mathrm{N}^{-1} \prod_{\mathrm{i}=1}^{\mathrm{r}}(\mathrm{N}-\mathrm{i}+1)
$$

onde $\mathrm{N} \geq \mathrm{r}$ e $\lambda>0$.

A densidade a posteriori marginal para $\mathrm{N}$ é obtida integrando-se a densidade a posteriori conjunta para $\mathrm{N}$ e $\lambda(3.15)$ em relação a $\lambda$, ou seja,

$$
\pi(\mathrm{N}) \propto \mathrm{N}^{-1} \prod_{\mathrm{i}=1}^{\mathrm{r}}(\mathrm{N}-\mathrm{i}+1) \int_{0}^{\infty} \lambda^{\mathrm{r}+\mathrm{m}-1} \mathrm{e}^{-\mathrm{N} \lambda \tau} \mathrm{d} \lambda \propto \frac{\prod_{\mathrm{i}=1}^{\mathrm{r}}(\mathrm{N}-\mathrm{i}+1)}{\mathrm{N}^{\mathrm{r}+\mathrm{m}+1}} .
$$


Podemos observar que

$$
\pi(\mathrm{N}) \propto \frac{\mathrm{L}_{\mathrm{p}}(\mathrm{N})}{\mathrm{N}}
$$

onde $\mathrm{L}_{\mathrm{p}}(\mathrm{N})$ é a função de verossimilhança profile de $\mathrm{N}$ (2.21).

Um fato importante a ser observado é que a densidade a posteriori marginal de $\mathrm{N}$ (3.16) existe se, e somente se, $\mathrm{m}>0$. Portanto, com os contadores incluídos no modelo, não teremos problemas de a posteriori marginal de $\mathrm{N}$ não existir. Essa é uma boa justificativa, do ponto de vista Bayesiano, para a inclusão dos contadores ao nosso modelo.

Usando a expressão (3.17) e o mesmo procedimento utilizado na seção 2.2.4 para obter o e.m.v. de $\mathrm{N}$, nós podemos encontrar a moda, $\hat{\mathrm{N}}_{0}$, de $\mathrm{N}$ :

Seja

$$
\mathrm{g}^{*}(\mathrm{~N})=\frac{\pi(\mathrm{N})}{\pi(\mathrm{N}-1)}=\left(1-\frac{1}{\mathrm{~N}}\right)^{\mathrm{r}+\mathrm{m}+1}+\frac{\mathrm{r}}{\mathrm{N}}, \mathrm{N}>\mathrm{r}
$$

Se $g^{*}(r+1)<1$, então $\hat{N}_{o}=r$. Se $g^{*}(r+1)<1$, então $\hat{N}_{o}=k$ se, e somente se, $\mathrm{h}^{* *}{ }_{\mathrm{r}, \mathrm{k}+1}<\mathrm{m}<\mathrm{h}_{\mathrm{r}, \mathrm{k}}^{* *}$, onde

$$
\mathrm{h}_{\mathrm{r}, \mathrm{k}}^{* *}=\frac{\log \left(1-\frac{\mathrm{r}}{\mathrm{k}}\right)}{\log \left(1-\frac{1}{\mathrm{k}}\right)}-(\mathrm{r}+1), \mathrm{k}>\mathrm{r}
$$


Para obter os resumos a posteriori, utilizaremos as densidades a posteriori condicionais de $\mathrm{N}$ e $\lambda$ requeridas para o algoritmo de Gibbs Sampling, que são dadas por:

$$
\begin{aligned}
& \lambda \mid \mathrm{N} \sim \Gamma[\mathrm{r}+\mathrm{m}, \mathrm{N} \tau], \\
& \pi(\mathrm{N} \mid \lambda) \propto \psi_{1}(\mathrm{~N}, \lambda),
\end{aligned}
$$

onde $\psi_{1}(\mathrm{~N}, \lambda)=\exp \left\{\sum_{\mathrm{i}=1}^{\mathrm{r}} \ln (\mathrm{N}-\mathrm{i}+1)-\ln (\mathrm{N})-\mathrm{N} \lambda \tau\right\}$

Podemos observar que a densidade a posteriori condicional de $\lambda$ tem distribuição Gama com média $[(\mathrm{r}+\mathrm{m}) / \mathrm{N} \tau]$ e pode ser gerada através do algoritmo de Gibbs Sampling. Como não identificamos uma distribuição conhecida para a densidade a posteriori condicional de $\mathrm{N}$, devemos então obtê-la através do algoritmo de Metropolis-Hastings.

\subsection{Análise Bayesiana do Modelo de Goel e Okumoto}

A função de verossimilhança para o modelo da Goel e Okumoto pode ser escrita como

$$
L(N, \lambda, p)=\lambda^{r}\left(\prod_{i=1}^{r}[N-p(i-1)]\right) \exp \left\{-\lambda\left[p \sum_{i=1}^{r} t_{(i)}+(N-r p) t_{(r)}\right]\right\}
$$


Assumindo que o pesquisador não tenha informação prévia a respeito dos parâmetros $\mathrm{N}$ e $\lambda$, vamos considerar uma priori não-informativa para esses parâmetros. Para $p$, consideramos uma priori informativa Beta, devido ao seu espaço paramétrico. Assim, temos:

$$
\begin{aligned}
& \mathrm{p}(\mathrm{N}) \propto \frac{1}{\mathrm{~N}}, \\
& \mathrm{p}(\lambda) \propto \frac{1}{\lambda}, \\
& \mathrm{p}(p) \sim \mathrm{B}\left[\mathrm{a}_{1}, \mathrm{~b}_{1}\right],
\end{aligned}
$$

onde $B\left[a_{1}, b_{1}\right]$ denota a distribuição Beta com média $\left[a_{1} /\left(a_{1}+b_{1}\right)\right]$ a $a_{1}$ e $b_{1}$ são constantes conhecidas.

Supondo independência a priori, a densidade a priori conjunta para $\mathrm{N}, \lambda$ e $p$ é dada por

$$
\mathrm{p}(\mathrm{N}, \lambda, p) \propto \frac{1}{\mathrm{~N} \lambda} \mathrm{p}^{\mathrm{a}_{1}-1}(1-\mathrm{p})^{\mathrm{b}_{1}-1}
$$

onde $\lambda>0,0 \leq p \leq 1$ e $\mathrm{N} \geq \mathrm{r}$.

A densidade a posteriori conjunta para $\mathrm{N}, \lambda$ e $p$, considerando-se a priori (3.22) e a função de verossimilhança (3.21), é dada por 


$$
\begin{aligned}
& \pi(\mathrm{N}, \lambda, p) \propto \frac{\lambda^{\mathrm{r}-1} \mathrm{p}^{\mathrm{a}_{1}-1}(1-\mathrm{p})^{\mathrm{b}_{1}-1}}{\mathrm{~N}}\left[\prod_{\mathrm{i}=1}^{\mathrm{r}}(\mathrm{N}-\mathrm{i}+1)\right] \mathrm{x} \\
& \mathrm{x} \exp \left\{-\lambda\left[\mathrm{p} \sum_{\mathrm{i}=1}^{\mathrm{r}} \mathrm{t}_{(\mathrm{i})}+(\mathrm{N}-\mathrm{r} \mathrm{p}) \mathrm{t}_{(\mathrm{r})}\right]\right\},
\end{aligned}
$$

onde $\lambda>0,0 \leq p \leq 1$ e $\mathrm{N} \geq \mathrm{r}$.

Considerando a densidade a posteriori conjunta (3.23), as densidades a posteriori condicionais para $\mathrm{N}, \lambda$ e $p$ requeridas para o algoritmo de Gibbs são indicadas por:

$$
\begin{aligned}
& \lambda \mid \mathrm{N}, p \sim \Gamma\left[\mathrm{r}, \mathrm{p} \sum_{\mathrm{i}=1}^{\mathrm{r}} \mathrm{t}_{(\mathrm{i})}+(\mathrm{N}-\mathrm{r} \mathrm{p}) \mathrm{t}_{(\mathrm{r})}\right], \\
& \pi(\mathrm{N} \mid \lambda, p) \propto \psi_{2}(\mathrm{~N}, \lambda, p), \\
& \pi(p \mid \mathrm{N}, \lambda) \propto \mathrm{p}^{\mathrm{a}_{1}-1}(1-\mathrm{p})^{\mathrm{b}_{1}-1} \psi_{3}(\mathrm{~N}, \lambda, p),
\end{aligned}
$$

onde $\psi_{2}(\mathrm{~N}, \lambda, p)=\exp \left\{\sum_{\mathrm{i}=1}^{\mathrm{r}} \ln [\mathrm{N}-\mathrm{p}(\mathrm{i}-1)]-\ln (\mathrm{N})-\lambda(\mathrm{N}-\mathrm{rp}) \mathrm{t}_{(\mathrm{r})}\right\} \mathrm{e}$

$\psi_{3}(\mathrm{~N}, \lambda, p)=\exp \left\{\sum_{\mathrm{i}=1}^{\mathrm{r}} \ln [\mathrm{N}-\mathrm{p}(\mathrm{i}-1)]-\lambda\left[\mathrm{p} \sum_{\mathrm{i}=1}^{\mathrm{r}} \mathrm{t}_{(\mathrm{i})}+(\mathrm{N}-\mathrm{rp}) \mathrm{t}_{(\mathrm{r})}\right]\right\}$. 
A densidade a posteriori condicional de $\lambda$ (que tem distribuição Gama) pode ser gerada através do algoritmo de Gibbs. As densidades a posteriori condicionais de $\mathrm{N}$ e $p$ podem ser geradas através do algoritmo de Metropolis-Hastings.

Para utilizarmos o algoritmo de Metropolis-Hastings é necessária a escolha de um núcleo de transição. Observando a densidade a posteriori condicional de $p$ (3.24), temos que o núcleo de transição é dado por uma distribuição Beta.

Para a densidade a posteriori condicional de N (3.24), não encontramos um núcleo de transição. Nesse caso, devemos utilizar um núcleo de transição (q) que seja simétrico, $\mathrm{q}(\theta, \phi)=\mathrm{q}(\phi, \theta)$, para todo $\operatorname{par}(\theta, \phi)$, de forma que a razão de teste (3.5) se reduza a

$$
\alpha(\theta, \phi)=\min \left\{1, \frac{\pi(\phi)}{\pi(\theta)}\right\}
$$

e dessa forma, não dependa de $\mathrm{q}$, implicando assim em uma simplificação computacional, que pode ser significativa.

\subsection{Análise Bayesiana do Modelo de Goel e Okumoto com Captura-Recaptura}

A função de verossimilhança para o modelo de Goel e Okumoto com capturarecaptura é dada por 


$$
L(N, \lambda, p) \propto \lambda^{r+m} \prod_{i=1}^{r}[N-p(i-1)] \exp \left\{-\lambda\left[N \tau+(1-p)\left(r \tau-\sum_{i=1}^{r} t_{(i)}\right)\right]\right\},
$$

onde $\mathrm{N} \geq \mathrm{r}, 0 \leq \mathrm{p} \leq 1, \lambda>0$.

\section{$1^{\circ}$ caso: $p$ conhecido}

A fim de formularmos a priori, nós consideramos o seguinte modelo hierárquico para $\mathrm{N}$ :

$$
\mathrm{N} \mid \mu \sim \mathrm{P}(\mu), \quad \mu \sim \Gamma\left(\mathrm{a}_{2}, \mathrm{~b}_{2}\right),
$$

onde $\mathrm{P}(\mu)$ denota a distribuição de Poisson com média $\mu, \Gamma\left(\mathrm{a}_{2}, \mathrm{~b}_{2}\right)$ denota a distribuição Gama com média $\left(a_{2} / b_{2}\right)$ e $a_{2}$ e $b_{2}$ são constantes conhecidas.

Quando temos um modelo hierárquico, os parâmetros das distribuições dos parâmetros são desconhecidos com uma distribuição conhecida. A densidade a priori hierárquica facilita a compreensão da escolha de determinada distribuição a priori para o parâmetro.

Com essa priori hierárquica, teremos uma priori Binomial Negativa para $\mathrm{N}$ com parâmetros $a_{2}$ e $\left[b_{2} /\left(b_{2}+1\right)\right]$ (ver apêndice 2) que é representada por

$$
\mathrm{p}(\mathrm{N})=\int_{0}^{\infty} \frac{\mathrm{e}^{-\mu} \mu^{\mathrm{N}}}{\mathrm{N} !} \frac{\mu^{\mathrm{a}_{2}-1} \mathrm{e}^{-\mathrm{b}_{2} \mu} \mathbf{b}_{2}^{\mathrm{a}_{2}}}{\Gamma\left(\mathrm{a}_{2}\right)} \mathrm{d} \mu \propto \frac{\Gamma\left(\mathrm{N}+\mathrm{a}_{2}\right)}{\mathrm{N} !\left(\mathrm{b}_{2}+1\right)^{\mathrm{N+a_{2 }}}} .
$$


Para $\lambda$, assumiremos uma priori Gama com parâmetros $a_{3}$ e $b_{3}$, ou seja,

$$
\mathrm{p}(\lambda) \propto \lambda^{\mathrm{a}_{3}-1} \exp \left\{-\mathrm{b}_{3} \lambda\right\} .
$$

Assim, a densidade a priori conjunta para $\mathrm{N}$ e $\lambda$ é dada por

$$
\mathrm{p}(\mathrm{N}, \lambda)=\frac{\Gamma\left(\mathrm{N}+\mathrm{a}_{2}\right) \lambda^{\mathrm{a}_{3}-1} \exp \left\{-\mathrm{b}_{3} \lambda\right\}}{\mathrm{N} !\left(\mathrm{b}_{2}+1\right)^{\mathrm{N}+\mathrm{a}_{2}}}
$$

onde $\mathrm{N} \geq \mathrm{r}, \lambda>0$.

Uma vez definido o modelo para os dados e a distribuição a priori, devemos combinar a informação prévia sobre os parâmetros com a informação contida nos dados utilizando a fórmula de Bayes, obtendo assim uma densidade a posteriori.

A densidade a posteriori conjunta para $\mathrm{N}$ e $\lambda$ ( $\mathrm{p}$ conhecido) é expressa por

$$
\begin{aligned}
\pi(\mathrm{N}, \lambda) & \propto \lambda^{\mathrm{r}+\mathrm{m}+\mathrm{a}_{3}-1} \prod_{\mathrm{i}=1}^{\mathrm{r}}[\mathrm{N}-\mathrm{p}(\mathrm{i}-1)] \frac{\Gamma\left(\mathrm{N}+\mathrm{a}_{2}\right)}{\mathrm{N} !\left(\mathrm{b}_{2}+1\right)^{\mathrm{N}+\mathrm{a}_{2}}} \mathrm{x} \\
& \times \exp \left\{-\lambda\left[\mathrm{b}_{3}+\mathrm{N} \tau+(1-\mathrm{p})\left(\mathrm{r} \tau-\sum_{\mathrm{i}=1}^{\mathrm{r}} \mathrm{t}_{(\mathrm{i})}\right)\right]\right\} .
\end{aligned}
$$

A densidade a posteriori marginal para $\mathrm{N}$ é obtida integrando-se a densidade a posteriori conjunta para $\mathrm{N}$ e $\lambda(3.29)$ em relação a $\lambda$. Assim temos que 


$$
\pi(N) \propto \frac{\prod_{i=1}^{r}[N-p(i-1)] \Gamma\left(N+a_{2}\right)}{N !\left(b_{2}+1\right)^{N+a_{2}}\left\{b_{3}+N \tau+(1-p)\left[r \tau-\sum_{i=1}^{r} t_{(i)}\right]\right\}^{r+m+a_{3}}}
$$

Se $a_{2}=a_{3}=b_{2}=b_{3}=0$ em (3.28), nós temos uma priori não-informativa para $\mathrm{N}$ e $\lambda$, indicada por

$$
\begin{gathered}
\mathrm{p}(\lambda) \propto \frac{1}{\lambda} \quad \text { e } \quad \mathrm{p}(\mathrm{N}) \propto \frac{1}{\mathrm{~N}} \\
\Rightarrow \quad \mathrm{p}(\mathrm{N}, \lambda) \propto \frac{1}{\mathrm{~N} \lambda}
\end{gathered}
$$

Com essa priori não-informativa, a densidade a posteriori conjunta é dada por

$$
\pi(N, \lambda) \propto \frac{\lambda^{\mathrm{r}+\mathrm{m}-1}}{\mathrm{~N}}\left(\prod_{\mathrm{i}=1}^{\mathrm{r}}[\mathrm{N}-\mathrm{p}(\mathrm{i}-1)]\right) \exp \left\{-\lambda\left[\mathrm{N} \tau+(1-\mathrm{p})\left(\mathrm{r} \tau-\sum_{\mathrm{i}=1}^{\mathrm{r}} \mathrm{t}_{(\mathrm{i})}\right)\right]\right\}
$$

e a densidade a posteriori marginal de $\mathrm{N}$ torna-se

$$
\pi(N) \propto \frac{\prod_{i=1}^{r}[N-p(i-1)]}{N\left\{N \tau+(1-p)\left[r \tau-\sum_{i=1}^{r} t_{(i)}\right]\right\}^{r+m}}
$$

onde $\mathrm{N} \geq \mathrm{r}$. 
Observe-se que a posteriori marginal de $\mathrm{N}$ (3.33) existe se, e somente se, $\mathrm{m}>0$. Podemos observar também que

$$
\pi(\mathrm{N}) \propto \frac{\mathrm{L}_{\mathrm{p}}(\mathrm{N})}{\mathrm{N}},
$$

onde $L_{p}(N)$ é a função de verossimilhança profile (2.64).

Usando (3.34), nós podemos encontrar a moda, $\hat{\mathrm{N}}_{\mathrm{o}}$, de $\mathrm{N}$ :

Seja

$$
\mathrm{g}^{* *}(\mathrm{~N})=\frac{\pi(\mathrm{N})}{\pi(\mathrm{N}-1)}=\left[1-\frac{1}{\mathrm{~N}+(1-\mathrm{p}) \mathrm{v}}\right]^{\mathrm{r}+\mathrm{m}}+1-\prod_{\mathrm{i}=2}^{\mathrm{r}}\left[1-\frac{1}{\mathrm{~N}-\mathrm{p}(\mathrm{i}-1)}\right]
$$

onde $v=\frac{r \tau-\sum_{i=1}^{r} t_{(i)}}{\tau}, N \geq r+1, r \geq 2$

Se $r=1$, então $\hat{N}_{o}=1$. Se $g^{* *}(r+1)<1$, então $\hat{N}_{o}=r$. Se $g^{* *}(r+1)<1$, então $\hat{\mathrm{N}}_{\mathrm{o}}=\mathrm{k}$ se, e somente se, $\mathrm{h}_{\mathrm{r}, \mathrm{k}+1}^{* * *}<\mathrm{m}<\mathrm{h}_{\mathrm{r}, \mathrm{k}}^{* * *}$, onde

$$
\mathrm{h}_{\mathrm{r}, \mathrm{k}}^{* * *}=\frac{\sum_{\mathrm{i}=2}^{\mathrm{r}} \log \left(1-\frac{1}{\mathrm{k}-\mathrm{p}(\mathrm{i}-1)}\right)}{\log \left\{1-\frac{1}{\mathrm{k}+(1-\mathrm{p}) \mathrm{v}}\right\}}-\mathrm{r} .
$$


Para obtermos os resumos a posteriori, utilizaremos as densidades a posteriori condicionais de $\mathrm{N}$ e $\lambda$, requeridas para o algoritmo de Gibbs Sampling.

Utilizando a densidade a posteriori conjunta (3.32), encontramos as densidades a posteriori condicionais de $\mathrm{N}$ e $\lambda$, que são dadas por:

$$
\begin{aligned}
& \lambda \mid \mathrm{N} \sim \Gamma\left[\mathrm{r}+\mathrm{m}, \mathrm{N} \tau+(1-\mathrm{p})\left(\mathrm{r} \tau-\sum_{\mathrm{i}=1}^{\mathrm{r}} \mathrm{t}_{(\mathrm{i})}\right)\right], \\
& \pi(\mathrm{N} \mid \lambda) \propto \psi_{4}(\mathrm{~N}, \lambda),
\end{aligned}
$$

onde $\psi_{4}(N, \lambda)=\exp \left\{\sum_{i=1}^{\mathrm{r}} \ln [N-p(i-1)]-\ln (N)-N \lambda \tau\right\}$.

De (3.37) podemos observar que $\mathrm{N}$ pode ser gerado utilizando-se o algoritmo de Metropolis-Hastings e $\lambda$, através do algoritmo de Gibbs Sampling.

\section{$2^{\circ}$ caso: $p$ desconhecido}

Quando assumimos p desconhecido, é necessária a escolha de uma priori para p. De acordo com o espaço paramétrico dos parâmetros, consideramos as seguintes densidades a priori para $\mathrm{N}, \lambda$ e $\mathrm{p}$ : 


$$
\begin{gathered}
\mathrm{N} \sim \mathrm{P}\left(\gamma_{1}\right), \\
\lambda \sim \Gamma\left[\mathrm{a}_{4}, \mathrm{~b}_{4}\right], \\
\mathrm{p} \sim \mathrm{B}\left[\mathrm{a}_{5}, \mathrm{~b}_{5}\right],
\end{gathered}
$$

onde $\Gamma\left[a_{4}, b_{4}\right]$ denota a distribuição Gama com média $\left(a_{4} / b_{4}\right), P\left(\gamma_{1}\right)$ denota a distribuição de Poisson com média $\gamma_{1}, \mathrm{~B}\left[\mathrm{a}_{5}, \mathrm{~b}_{5}\right]$ denota a distribuição Beta com média $\left[a_{5} /\left(a_{5}+b_{5}\right)\right]$ e $a_{4}, b_{4}, a_{5}, b_{5}$ e $\gamma_{1}$ são constantes conhecidas.

Assumindo que $\mathrm{N}, \lambda$ e $p$ são independentes, a densidade a priori conjunta para esses parâmetros é expressa por

$$
\mathrm{p}(\mathrm{N}, \lambda, p) \propto \frac{\gamma_{1}^{\mathrm{N}} \exp \left\{-\gamma_{1}\right\}}{\mathrm{N} !} \lambda^{\mathrm{a}_{4}-1} \exp \left\{-\lambda \mathrm{b}_{4}\right\} \mathrm{p}^{\mathrm{a}_{5}-1}(1-\mathrm{p})^{\mathrm{b}_{5}-1}
$$

onde $\mathrm{N} \geq \mathrm{r}, \lambda>0$ e $0 \leq p \leq 1$.

A densidade a posteriori conjunta para $\mathrm{N}, \lambda$ e $p$ é dada por

$$
\begin{array}{r}
\pi(\mathrm{N}, \lambda, p) \propto \frac{\gamma_{1}^{\mathrm{N}} \lambda^{\mathrm{r}+\mathrm{m}+\mathrm{a}_{4}-1} \prod_{\mathrm{i}=1}^{\mathrm{r}}[\mathrm{N}-\mathrm{p}(\mathrm{i}-1)]}{\mathrm{N} !} \mathrm{p}^{\mathrm{a}_{5}-1}(1-\mathrm{p})^{\mathrm{b}_{\mathrm{s}}-1} \mathrm{x} \\
\quad \mathrm{x} \exp \left\{-\gamma_{1}-\lambda\left[\mathrm{b}_{4}+\mathrm{N} \tau+(1-\mathrm{p})\left(\mathrm{r} \tau-\sum_{\mathrm{i}=1}^{\mathrm{r}} \mathrm{t}_{(\mathrm{i})}\right)\right]\right\} .
\end{array}
$$


Neste caso, as densidades a posteriori marginais para o algoritmo de Gibbs com Metropolis são dadas por:

$$
\begin{aligned}
& \lambda \mid \mathrm{N}, p \sim \Gamma\left[\mathrm{r}+\mathrm{m}+\mathrm{a}_{4}, \mathrm{~b}_{4}+\mathrm{N} \tau+(1-\mathrm{p})\left(\mathrm{r} \tau-\sum_{\mathrm{i}=1}^{\mathrm{r}} \mathrm{t}_{(\mathrm{i})}\right)\right], \\
& \pi(\mathrm{N} \mid \lambda, p) \propto \frac{\gamma_{1}^{\mathrm{N}} \exp \left\{-\gamma_{1}\right\}}{\mathrm{N} !} \psi_{5}(\mathrm{~N}, \lambda, p), \\
& \pi(p \mid \mathrm{N}, \lambda) \propto \mathrm{p}^{\mathrm{a}_{s}-1}(1-\mathrm{p})^{\mathrm{b}_{5}-1} \psi_{6}(\mathrm{~N}, \lambda, p),
\end{aligned}
$$

onde $\psi_{5}(\mathrm{~N}, \lambda, p)=\exp \left\{\sum_{\mathrm{i}=1}^{\mathrm{r}} \ln [\mathrm{N}-\mathrm{p}(\mathrm{i}-1)]-\mathrm{N} \lambda \tau\right\} \mathrm{e}$

$\psi_{6}(\mathrm{~N}, \lambda, p)=\exp \left\{\sum_{\mathrm{i}=1}^{\mathrm{r}} \ln [\mathrm{N}-\mathrm{p}(\mathrm{i}-1)]+\lambda \mathrm{p}\left(\mathrm{r} \tau-\sum_{\mathrm{i}=1}^{\mathrm{r}} \mathrm{t}_{(\mathrm{i})}\right)\right\}$

Podemos observar que $\mathrm{N}$ e $p$ podem ser gerados utilizando-se o algoritmo de Metropolis-Hastings e $\lambda$, utilizando-se o algoritmo de Gibbs Sampling. 


\section{CAPÍTULO 4}

\section{UMA ANÁLISE DA DISTRIBUIÇÃO A POSTERIORI DE N VIA MEDIDAS DE DIVERGÊNCIA}

\subsection{Introdução}

Neste capítulo, nós compararemos a distribuição a posteriori marginal de $\mathrm{N}$ (3.33), para diferentes valores de p. O que queremos saber é se mudanças na probabilidade de corrigir um erro quando este é encontrado correspondem a mudanças substanciais nos resultados da análise.

$\mathrm{Na}$ análise Bayesiana em particular, observados os dados, nós podemos considerar a distribuição a posteriori do modelo ou a distribuição preditiva de futuras observações. Nossa idéia é fazer uma análise da sensibilidade de $p$, baseada na distribuição a posteriori marginal de $\mathrm{N}$.

Para compararmos distribuições resultantes de diferentes escolhas dos parâmetros do modelo, nós precisamos de uma medida geral de diferenças entre duas distribuições. Nós consideraremos a medida de divergência (Ф-divergência) definida por Csiszár (1967) para medir a discrepância entre duas posteriores $\pi$ e $\pi_{0}$. 
Muitas escolhas de $\Phi$ são dadas em Dey e Birmiwal (1993). Por exemplo, $\Phi(\mathrm{x})=-\ln (\mathrm{x})$ define a divergência de Kullback-Leibler; $\Phi(\mathrm{x})=\frac{1}{2}|\mathrm{x}-1|$, a distância da Variação Total ou norma $\mathrm{L}_{1} ; \Phi(\mathrm{x})=(\mathrm{x}-1)^{2}$, a divergência qui-quadrado e $\Phi(\mathrm{x})=(\sqrt{\mathrm{x}}-1)^{2} / 2$, a distância de Hellinger.

Segundo Johnson e Geisser (1983), nós devemos usar a divergência de Kullback-Leibler, que é uma medida de divergência natural que reflete o específico interesse do investigador. Peng e Dey (1995) recomendam usar a distância da Variação Total, devido à facilidade de interpretação.

Nosso objetivo, então, será comparar a distribuição a posteriori marginal de $\mathrm{N}$, (3.33), para diferentes valores de p, usando as medidas de divergência de KullbackLeibler (KL) e da Variação Total (VT).

\subsection{Comparando as Posteriores Marginais de N ( p conhecido)}

Suponha-se que tenhamos feito toda a nossa análise admitindo que, à medida que os erros são encontrados, eles são corrigidos com probabilidade $p=1$, e portanto, a correção desse erro não fará surgir outro; e suponha-se que o pesquisador não tenha tanta certeza de que $\mathrm{p}=1$ e resolva testar outros valores de $\mathrm{p}$, mantendo o seu conjunto de dados obtido com $\mathrm{p}=1$.

Feito isso, o que queremos descobrir é o seguinte: se eu suponho $\mathrm{p}=1$, mas acho que ele possa ser $\mathrm{p}=0.5$ ou outro valor entre 0.1 e 0.9 , isso estaria afetando 
muito a minha distribuição a posteriori marginal de $\mathrm{N}$ ?

Seja $\pi_{0}=\pi_{0}(N \mid$ dados $)$ a distribuição a posteriori de $N(3.33)$ com $p=1$, dada por

$$
\pi_{0}(N \mid \text { dados }) \propto \frac{\prod_{i=1}^{\mathrm{r}}[N-(\mathrm{i}-1)]}{\mathrm{N}[\mathrm{N} \tau]^{\mathrm{r}+\mathrm{m}}}, \mathrm{N} \geq \mathrm{r}
$$

e $\pi=\pi(\mathrm{N} \mid$ dados $)$ a distribuição a posteriori de $\mathrm{N}$ para $0<\mathrm{p}<1$, obtida em (3.33), dada por

$$
\pi(N \mid \text { dados }) \propto \frac{\prod_{i=1}^{r}[N-p(i-1)]}{N\left[N \tau+(1-p)\left(r \tau-\sum_{i=1}^{r} t_{(i)}\right)\right]^{r+m}}, N \geq r .
$$

Segundo Csiszár (1967), nós podemos, em geral, definir a Ф-divergência entre as posteriori $\pi$ e $\pi_{0}$ como

$$
\mathrm{D}_{\Phi}=\mathrm{D}\left(\pi_{\mathrm{o}}, \pi\right)=\sum \Phi\left(\frac{\pi}{\pi_{0}}\right) \pi_{0}
$$

no caso de $\pi$ e $\pi_{0}$ serem distribuições discretas, e

$$
\mathrm{D}_{\Phi}=\mathrm{D}\left(\pi_{\mathrm{o}}, \pi\right)=\int \Phi\left(\frac{\pi}{\pi_{0}}\right) \pi_{0} \mathrm{~d} \theta
$$


no caso de $\pi$ e $\pi_{0}$ serem distribuições contínuas, onde $\theta$ é algum parâmetro do modelo.

\subsection{Calibração das $\Phi$-Divergências}

Como todas as distâncias são pequenas, como saber quais valores são significantes a ponto de podermos afirmar que as densidades a posteriori são realmente diferentes? Temos, então, que estabelecer algum critério que nos diga acima de qual valor existe realmente alguma diferença entre as distribuições a posteriori. Este critério que nos dá a significância da perturbação é chamado de calibração.

Para obter essa calibração, Peng e Dey (1995) consideraram uma moeda viciada, cuja probabilidade de sucesso é p. Então a $\Phi$-divergência entre a moeda não-viciada e a viciada é dada por

$$
D\left(f_{o}, f_{1}\right)=\int \Phi\left(\frac{f_{1}(x)}{f_{o}(x)}\right) f_{o}(x) d x
$$

onde $f_{0}(x)=\frac{1}{2}$ e $f_{1}(x)=p^{x}(1-p)^{1-x}, x=0,1$.

$\operatorname{Se} D\left(f_{o}, f_{l}\right)=d$, então d satisfaz a seguinte equação

$$
\mathrm{d}=\frac{\Phi(2 p)+\Phi(2(1-p))}{2}
$$


Assim, a divergência entre duas posteriores pode ser associada ao vício de uma moeda.

\subsubsection{Calibração da Distância da Variação Total}

Substituindo $\Phi(\mathrm{x})=\frac{1}{2}|\mathrm{x}-1|$ (distância da Variação Total) em (4.6), encontramos $d=\frac{1}{2}|1-2 p|$.

$$
\begin{aligned}
\mathrm{d} & =\frac{\Phi(2 \mathrm{p})+\Phi(2(1-\mathrm{p}))}{2}=\frac{\frac{1}{2}|2 \mathrm{p}-1|+\frac{1}{2}|2(1-\mathrm{p})-1|}{2} \\
& =\frac{\frac{1}{2}|1-2 \mathrm{p}|+\frac{1}{2}|1-2 \mathrm{p}|}{2}=\frac{1}{2}|1-2 \mathrm{p}|
\end{aligned}
$$

O gráfico de $d=\frac{1}{2}|1-2 p|$, para $0 \leq p \leq 1$, é mostrado na figura (4.1).

d

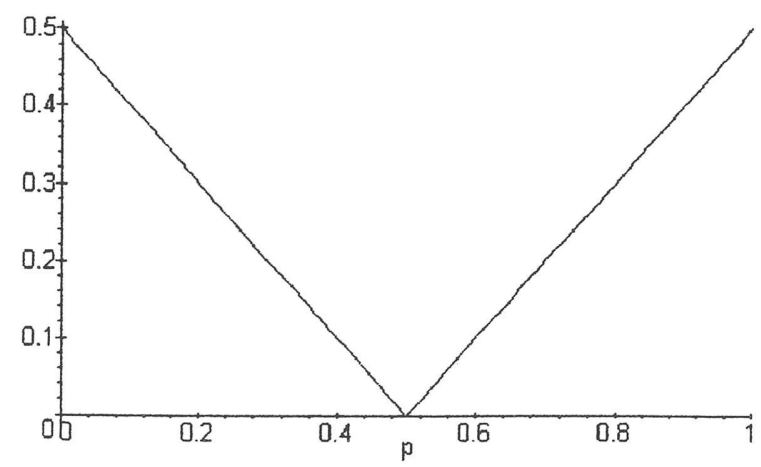

Figura 4.1: Gráfico de $\mathrm{d}=\frac{1}{2}|1-2 \mathrm{p}|, 0 \leq \mathrm{p} \leq 1$. 
Observe-se que $\mathrm{d}=\frac{1}{2}$ para $\mathrm{p}=0$ ou $1, \mathrm{e} \mathrm{d}=0$ para $\mathrm{p}=\frac{1}{2}$. A função $\mathrm{d}$ é simétrica e seu mínimo ocorre quando $f_{o}=f_{1}$. Vamos considerar somente $p>0.5$, tal que $\mathrm{d} \in[0,0.5]$. Para $\mathrm{p} \leq 0.5, \mathrm{~d}$ terá as mesmas propriedades devido à simetria da função $d$.

Assim, $\mathrm{p}=0.55$ corresponde a $\mathrm{d}=0.05$ indicando que estamos comparando uma moeda honesta com uma quase honesta, e $\mathrm{p}=0.9$ corresponde a $\mathrm{d}=0.4$, ou seja, estamos comparando uma moeda não-viciada com uma moeda viciada.

Estendendo para o nosso caso, onde $\pi_{0}$ seria a moeda honesta e $\pi$ a moeda viciada, teremos então a seguinte escala para a distância da Variação Total:

$$
\begin{aligned}
& \mathrm{VT}\left(\pi_{0}, \pi\right)<0.10, \text { temos uma pequena diferença entre } \pi_{0} \text { e } \pi, \\
& \operatorname{VT}\left(\pi_{0}, \pi\right) \in[0.1,0.25] \text {, temos uma diferença moderada entre } \pi_{0} \text { e } \pi, \\
& \operatorname{VT}\left(\pi_{0}, \pi\right)>0.25 \text {, temos uma grande diferença entre } \pi_{0} \text { e } \pi .
\end{aligned}
$$

Os intervalos obtidos são a nossa calibração da distância da Variação Total. Com esses intervalos fica fácil dar uma interpretação para as distâncias obtidas quando a forma da nossa distribuição a posteriori não é conhecida.

\subsubsection{Calibração da Divergência de Kullback-Leibler}

Segundo McCulloch (1989), um critério para decidirmos o quão distantes estão as densidades a posteriori, é utilizar uma calibração. Seja $B(\mathrm{p})$ denotando a 
distribuição de Bernoulli que atribui probabilidade $\mathrm{p}$ a um evento. Dado $\mathrm{KL}\left(\pi_{\mathrm{o}}, \pi\right)=\mathrm{k}$, nós encontramos $\mathrm{p}(\mathrm{k})$, tal que $\mathrm{KL}(B(1 / 2), B(\mathrm{p}(\mathrm{k})))=\mathrm{k}$. O número $\mathrm{p}(\mathrm{k})$ é nossa calibração de $\mathrm{k}$.

A calibração nos diz que, quando medida pela divergência de KullbackLeibler, a diferença $\mathrm{k}$ entre $\pi_{\mathrm{o}}$ e $\pi$ é a mesma que entre $B(1 / 2)$ e $B(\mathrm{p}(\mathrm{k}))$. Esta última divergência, por se tratar de uma distribuição conhecida (Bernoulli), servirá de base para compararmos distribuições desconhecidas. Observe-se que esta é a mesma idéia de comparar uma moeda honesta com uma moeda viciada vista na seção 4.3.

Como $\mathrm{KL}[B(1 / 2), B(\mathrm{p}(\mathrm{k}))]=-\ln (4 \mathrm{p}(1-\mathrm{p})) / 2$, nós escolhemos a solução na qual $\mathrm{p}(\mathrm{k})$ está entre 0.5 e 1.0 , que é $\mathrm{p}(\mathrm{k})=\left(1+\left(1-\mathrm{e}^{2 \mathrm{k}}\right)^{1 / 2}\right) / 2$. Dada esta restrição, a função $\mathrm{p}(\mathrm{k})$ é inversível, ou seja, temos uma correspondência um-a-um entre os valores $\mathrm{p}(\mathrm{k}) \mathrm{e} \mathrm{k}$.

$$
\begin{aligned}
\mathrm{KL}(B(1 / 2), B(\mathrm{p})) & =\sum_{\mathrm{x}=0}^{1}-\ln \left(\frac{\mathrm{B}(\mathrm{p})}{\mathrm{B}(1 / 2)}\right) \mathrm{B}(1 / 2)=-\frac{1}{2} \sum_{\mathrm{x}=0}^{1} \ln \left(2 \mathrm{p}^{\mathrm{x}}(1-\mathrm{p})^{1-\mathrm{x}}\right) \\
& =-\frac{1}{2}[\ln (2 \mathrm{p})+\ln (2(1-\mathrm{p}))]=-\frac{1}{2} \ln [4 \mathrm{p}(1-\mathrm{p})]
\end{aligned}
$$

Fazendo $-\frac{1}{2} \ln [4 p(1-p)]=k$, teremos $4 p(1-p)-e^{-2 k}=0$ e uma das duas soluções é $\mathrm{p}(\mathrm{k})=\left(1+\left(1-\mathrm{e}^{2 \mathrm{k}}\right)^{1 / 2}\right) / 2$. Na figura (4.2), temos o gráfico de $\mathrm{p}(\mathrm{k})$ versus $\mathrm{k}$. 


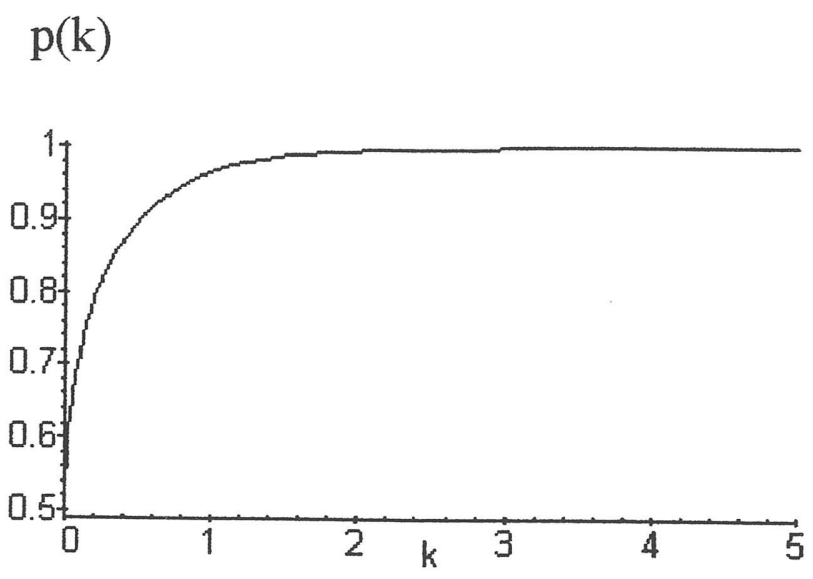

Figura 4.2: Gráfico de $\mathrm{p}(\mathrm{k})$ versus $\mathrm{k}$

Observe-se que, se substituirmos $\Phi=-\ln (\mathrm{x})$ (divergência de KullbackLeibler) em (4.6) e fizermos $d=k$, encontramos $k=-\ln (4 p(1-p)) / 2$.

A tabela (4.1) mostra alguns valores de $\mathrm{k}$ e os correspondentes valores de $\mathrm{p}(\mathrm{k})$. Essa tabela nos permite fazer uma comparação dos valores obtidos como se estivéssemos comparando duas distribuições de Bernoulli, $B(0.5)$ e $B(\mathrm{p}(\mathrm{k}))$.

\begin{tabular}{|c|c|}
\hline $\mathbf{k}$ & $\mathbf{p}(\mathbf{k})$ \\
\hline 0.00 & 0.50 \\
0.005 & 0.55 \\
0.02 & 0.60 \\
0.05 & 0.65 \\
0.09 & 0.70 \\
0.14 & 0.75 \\
0.22 & 0.80 \\
0.34 & 0.85 \\
0.51 & 0.90 \\
0.83 & 0.95 \\
\hline
\end{tabular}

Tabela 4.1: Calibração da divergência de KL. 
Por exemplo, se $\mathrm{p}(\mathrm{k})=0.99$, então $\pi$ e $\pi_{\mathrm{o}}$ são completamente diferentes. Se $\mathrm{p}(\mathrm{k})=0.501$, então $\pi$ e $\pi_{\mathrm{o}}$ são similares.

Como exemplo, suponha-se que $\pi_{\mathrm{o}}$ tenha uma distribuição Normal com média 0 e variância 5 e que $\pi$ tenha uma distribuição Normal com média 2 e variância 6 .

$$
\begin{aligned}
& \pi_{0}=\frac{1}{\sqrt{2 \pi} \sqrt{5}} \mathrm{e}^{\frac{-\mathrm{x}^{2}}{10}}, \quad-\infty \leq \mathrm{x} \leq \infty . \\
& \pi=\frac{1}{\sqrt{2 \pi} \sqrt{6}} \mathrm{e}^{\frac{-(\mathrm{x}-2)^{2}}{12}}, \quad-\infty \leq \mathrm{x} \leq \infty .
\end{aligned}
$$

A divergência de Kullback-Leibler é dada por

$$
\mathrm{KL}\left(\pi_{0}, \pi\right)=\int_{-\infty}^{\infty} \frac{-\sqrt{10} \mathrm{e}^{\left(-\frac{1}{10} \mathrm{x}^{2}\right)} \ln \left(\frac{1}{6} \frac{\sqrt{3} \mathrm{e}^{\left(-\frac{1}{12}(\mathrm{x}-2)^{2}\right)} \sqrt{10}}{\mathrm{e}^{\left(-\frac{1}{10} \mathrm{x}^{2}\right)}}\right)}{10 \sqrt{\pi}} \mathrm{dx}=0.34 .
$$

Como saber se a distância obtida 0.34 é grande ou não? Pela tabela (4.1), para $\mathrm{k}=0.34, \mathrm{p}(\mathrm{k})=0.85$. Isto significa que, se a variável aleatória é de fato uma $\mathrm{N}(0,5)$ mas chegamos em uma $\mathrm{N}(2,6)$, estamos então obtendo distribuicões bem diferentes, pois comparar uma $\mathrm{N}(0,5)$ com uma $\mathrm{N}(2,6)$ é equivalente a compararmos uma $\mathrm{B}(0.5)$ com uma $\mathrm{B}(0.85)$, que são muito diferentes. 


\subsection{Um Exemplo}

Vamos considerar os seguintes valores hipotéticos para os cálculos das medidas de divergência:

$$
\mathrm{r}=5, \tau=1, \mathrm{~m}=2 \text { e } \sum_{\mathrm{i}=1}^{5} \mathrm{t}_{(\mathrm{i})}=1
$$

Para esses valores, teremos as seguintes distribuições a posteriori marginais de $\mathrm{N}$ :

$$
\begin{gathered}
\pi_{0}(N \mid \text { dados }) \propto \frac{\prod_{i=1}^{5}[N-(i-1)]}{N[N]^{7}}=\frac{\prod_{i=1}^{4}[N-i]}{N^{7}}, \\
\pi(N \mid \text { dados }) \propto \frac{\prod_{i=1}^{5}[N-p(i-1)]}{N[N+(1-p)(5-1)]^{7}}=\frac{\prod_{i=1}^{4}[N-i p]}{[N+4(1-p)]^{7}} .
\end{gathered}
$$

As divergências de Kullback-Leibler e Variação Total entre $\pi_{0}$ e $\pi$ são dadas respectivamente por:

$$
\begin{aligned}
& \mathrm{KL}\left(\pi_{0}, \pi\right)=\sum_{\mathrm{N} \geq 5}-\ln \left(\frac{\pi}{\pi_{0}}\right) \pi_{0}, \\
& \operatorname{VT}\left(\pi_{0}, \pi\right)=\sum_{\mathrm{N} \geq 5} \frac{1}{2}\left|\left(\frac{\pi}{\pi_{0}}\right)-1\right| \pi_{0} .
\end{aligned}
$$


Note-se que $\operatorname{KL}\left(\pi_{0}, \pi\right) \neq \operatorname{KL}\left(\pi, \pi_{0}\right), \operatorname{VT}\left(\pi_{0}, \pi\right) \neq \operatorname{VT}\left(\pi, \pi_{0}\right)$ em geral; $\operatorname{KL}\left(\pi_{0}\right.$, $\pi)$ e $\operatorname{VT}\left(\pi_{0}, \pi\right)$ são sempre positivos, $\operatorname{KL}\left(\pi_{0}, \pi_{0}\right)=0$ e $\operatorname{VT}\left(\pi_{0}, \pi_{0}\right)=0$.

Feitos os cálculos, teremos a tabela (4.2) e o gráfico das distâncias entre as densidades a posteriori marginais de $\mathrm{N}$ (figura 4.3).

\begin{tabular}{|c|c|c|c|c|c|}
\hline $\mathbf{p}$ & $\mathbf{0 . 1 0}$ & $\mathbf{0 . 3 0}$ & $\mathbf{0 . 5 0}$ & $\mathbf{0 . 8 0}$ & $\mathbf{1 . 0}$ \\
\hline KL & 0.037 & 0.024 & 0.014 & 0.003 & 0.00 \\
\hline VT & 0.117 & 0.095 & 0.073 & 0.034 & 0.00 \\
\hline
\end{tabular}

Tabela 4.2 : Distâncias de KL e VT.

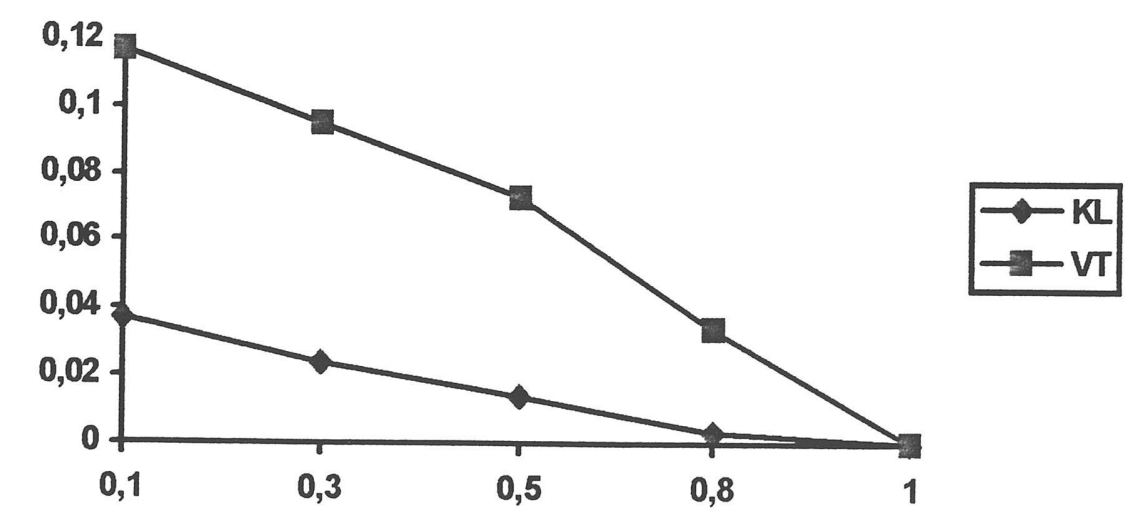

Figura 4.3: Gráfico da divergências de KL e VT

Observamos, pelo gráfico acima, que à medida que os valores de $\mathrm{p}$ aumentam, ou seja, quanto maior a probabilidade de corrigir um erro, menores serão as distâncias entre as distribuições a posteriori marginais de $\mathrm{N}$. 
Com a tabela para a calibração de KL e com os intervalos para VT, podemos agora tirar algumas conclusões deste exemplo hipotético. Para $\mathrm{p}=0.30, \mathrm{KL}\left(\pi_{\mathrm{o}}, \pi\right)=$ 0.024 , o que equivaleria a comparar uma $B(0.5)$ com uma $B(0.61)$; e para $\mathrm{p}=0.10$, $\mathrm{KL}\left(\pi_{0}, \pi\right)=0.037$, ou seja, estaríamos comparando uma $B(0.5)$ com uma $B(0.63)$.

Portanto, utilizando a distância de Kullback-Leibler, podemos dizer para esses dados hipotéticos que as distribuições $\pi_{0}$ e $\pi$ são quase similares. Utilizando a escala de Peng e Dey neste exemplo, temos uma diferença moderada entre as densidades a posteriori para $\mathrm{p}=0.10\left(\mathrm{VT}\left(\pi_{0}, \pi\right)=0.117\right)$. 


\section{CAPÍTULO 5}

\section{EXEMPLOS DE APLICAÇÃO}

\subsection{Exemplo Considerando o Modelo de Jelinski e Moranda}

Na tabela (5.1), temos um conjunto de dados apresentados por Jelinski e Moranda (1972). Eles consistem no número de dias entre as 26 falhas $(r=26)$ que ocorreram durante a fase de produção de um software (NTDS - Naval tactical data system).

\begin{tabular}{|crc|ccc|ccc|}
\hline $\mathbf{i}$ & $\mathbf{w}_{\mathbf{i}}$ & $\mathbf{t}_{(\mathbf{i})}$ & $\mathbf{i}$ & $\mathbf{w}_{\mathbf{i}}$ & $\mathbf{t}_{(\mathbf{i})}$ & $\mathbf{i}$ & $\mathbf{w}_{\mathbf{i}}$ & $\mathbf{t}_{(\mathbf{i})}$ \\
\hline 1 & 9 & 9 & 10 & 7 & 70 & 19 & 6 & 104 \\
2 & 12 & 21 & 11 & 1 & 71 & 20 & 1 & 105 \\
3 & 11 & 32 & 12 & 6 & 77 & 21 & 11 & 116 \\
4 & 4 & 36 & 13 & 1 & 78 & 22 & 33 & 149 \\
5 & 7 & 43 & 14 & 9 & 87 & 23 & 7 & 156 \\
6 & 2 & 45 & 15 & 4 & 91 & 24 & 91 & 247 \\
7 & 5 & 50 & 16 & 1 & 92 & 25 & 2 & 249 \\
8 & 8 & 58 & 17 & 3 & 95 & 26 & 1 & 250 \\
9 & 5 & 63 & 18 & 3 & 98 & & & \\
\hline
\end{tabular}

Tabela 5.1: Dados NTDS $\left(w_{i}=t_{(i)}-t_{(i-1)}\right)$ 


\section{Análise Clássica}

Considerando-se o conjunto de dados da tabela (5.1), os estimadores de máxima verossimilhança para os parâmetros são dados por:

$$
\begin{aligned}
& \hat{\mathrm{N}}=31.21553, \\
& \hat{\lambda}=0.00685 .
\end{aligned}
$$

$\mathrm{O}$ valor de $\hat{\mathrm{N}}$ foi obtido utilizando-se o método iterativo de Newton (ver apêndice 1).

A matriz de informação de Fisher (2.12) para $\mathrm{N}$ e $\lambda$ calculada em $(\hat{N}, \hat{\lambda})$, é dada por

$$
\mathrm{I}(\mathrm{N}, \lambda)=\left[\begin{array}{cc}
0.14299 & 249.98425 \\
249.98425 & 554105.1734
\end{array}\right]
$$

A inversa da matriz de informação de Fisher é a matriz de variância e covariância assintótica de $\hat{\mathrm{N}}$ e $\hat{\lambda}$, que é expressa por

$$
\mathrm{I}^{-1}(\hat{\mathrm{N}}, \hat{\lambda})=\left[\begin{array}{cc}
33.09909 & -0.01493 \\
-0.01493 & 0.00001
\end{array}\right]
$$


Intervalos de confiança $95 \%$ aproximados para $\mathrm{N}$ e $\lambda$, considerando-se a aproximação Normal assintótica (2.10) dos estimadores de máxima verossimilhança $\hat{\mathrm{N}}$ e $\hat{\lambda}$, são dados por:

$$
\begin{aligned}
& (19.9393<\mathrm{N}<42.49176), \\
& (0.00112<\lambda<0.01258) .
\end{aligned}
$$

Uma outra alternativa para obter o estimador de $\mathrm{N}$ é maximizar $\mathrm{L}_{\mathrm{p}}(\mathrm{N})$, a função de verossimilhança profile (2.8). Neste exemplo, os valores de $g(N)$ (2.9) para $\mathrm{N}=27, \ldots, 35$ são dados abaixo.

$$
\begin{array}{ll}
\mathrm{g}(27)=2.25 & \mathrm{~g}(32)=0.99 \\
\mathrm{~g}(28)=1.45 & \mathrm{~g}(33)=0.97 \\
\mathrm{~g}(29)=1.20 & \mathrm{~g}(34)=0.96 \\
\mathrm{~g}(30)=1.09 & \mathrm{~g}(35)=0.95 \\
\mathrm{~g}(31)=1.03 &
\end{array}
$$

Como $\mathrm{g}(31)>1$ e $\mathrm{g}(32)<1$, então $\hat{\mathrm{N}}_{\mathrm{p}}=31$ e $\hat{\lambda}=0.00695$. Observe-se que estes estimadores são próximos aos obtidos anteriormente $(\hat{\mathrm{N}}=31.21553$, $\hat{\lambda}=0.00685)$.

Para comprovar que a função $\mathrm{L}_{\mathrm{p}}(\mathrm{N})$ é maximizada com $\hat{\mathrm{N}}_{\mathrm{p}}=31$, observe-se o gráfico da função de verossimilhança profile mostrado na figura (5.1). 


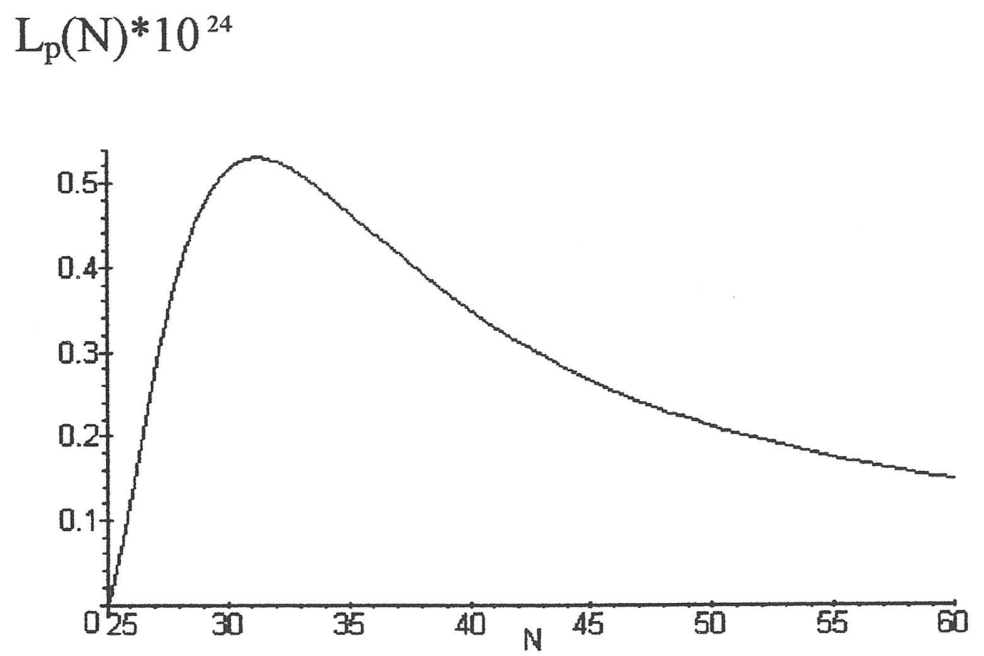

Figura 5.1 - Gráfico da profile de N

\section{Análise Bayesiana}

Como visto no capítulo 3, em 'Análise Bayesiana', as inferências sobre os parâmetros de interesse são baseadas nas suas densidades a posteriori marginais. As densidades a priori para os parâmetros $\mathrm{N}$ e $\lambda$ são dadas por:

$$
\begin{gathered}
\lambda \sim \Gamma[5.49,802.11], \\
\mathrm{N} \sim \mathrm{P}(31),
\end{gathered}
$$

onde $\mathrm{a}=5.49$ e $\mathrm{b}=802.11$ foram obtidos através do sistema de equações

$$
\left\{\begin{array}{l}
\mathrm{E}(\lambda)=\mathrm{a} / \mathrm{b}=\hat{\lambda} \\
\mathrm{V}(\lambda)=\mathrm{a} / \mathrm{b}^{2}=\mathrm{I}_{22}^{-1}(\mathrm{~N}, \lambda)
\end{array},\right.
$$


e $\gamma=31$ foi obtido a partir de $E(N)=\gamma=\hat{N}$.

Com os dados da tabela (5.1), temos que $r=26, N^{\prime}=N-26, t_{(r)}=250$ e $\sum_{\mathrm{i}=1}^{\mathrm{r}} \mathrm{t}_{(\mathrm{i})}=2492$. Assim, as densidades a posteriori condicionais (3.12) requeridas para o algoritmo de Gibbs Sampling são dadas por:

$$
\begin{gathered}
\lambda \mid \mathrm{N}^{\prime} \sim \Gamma\left[31.49,3294.11+250 \mathrm{~N}^{\prime}\right], \\
\mathrm{N}^{\prime} \mid \lambda \sim \mathrm{P}[31 \exp \{-250 \lambda\}] .
\end{gathered}
$$

Considerando as distribuições condicionais requeridas para o algoritmo de Gibbs, geramos cinco cadeias com 2000 iterações cada e, em cada cadeia, utilizamos diferentes valores iniciais para $\mathrm{N}^{\prime} \mathrm{e} \lambda$.

Para selecionarmos a amostra, desprezamos os primeiros 1000 valores gerados em cada cadeia e os valores restantes foram selecionados de $5 \mathrm{em} 5$, totalizando uma amostra de tamanho 1000 .

Os primeiros 1000 valores em cada cadeia foram desprezados pelo fato de que, à medida que o número de iterações aumenta, a cadeia se aproxima de sua condição de equilíbrio e, dessa maneira, a convergência é atingida nas últimas iterações da cadeia. 
Selecionamos a amostra de 5 em 5 , de forma a garantir uma amostra nãocorrelacionada e independente. Nas figuras (5.2) e (5.3), temos as funções de autocorrelação das amostras de $\mathrm{N}^{\prime}$ e $\lambda$.

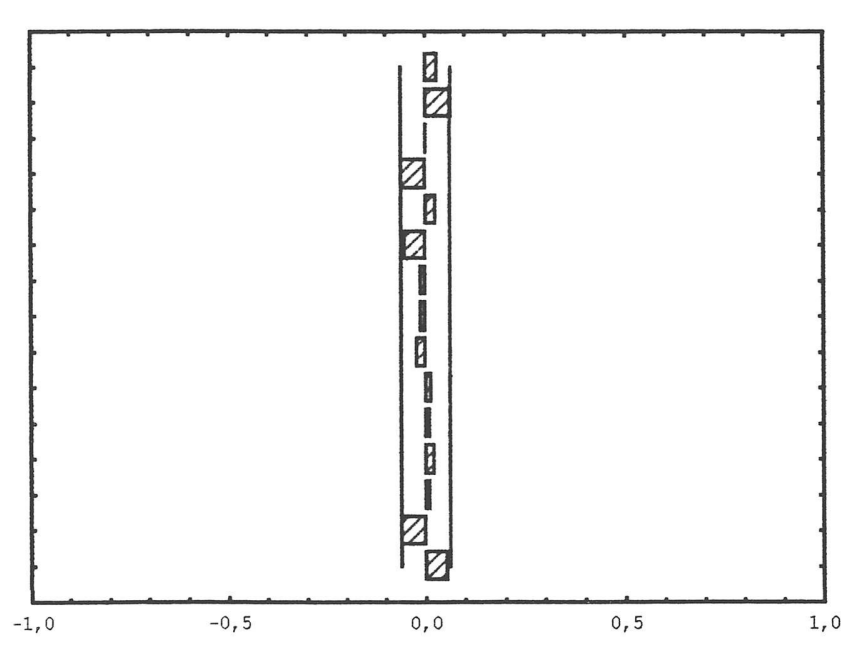

Figura 5.2: Função de autocorrelação de $\mathrm{N}^{\prime}$.

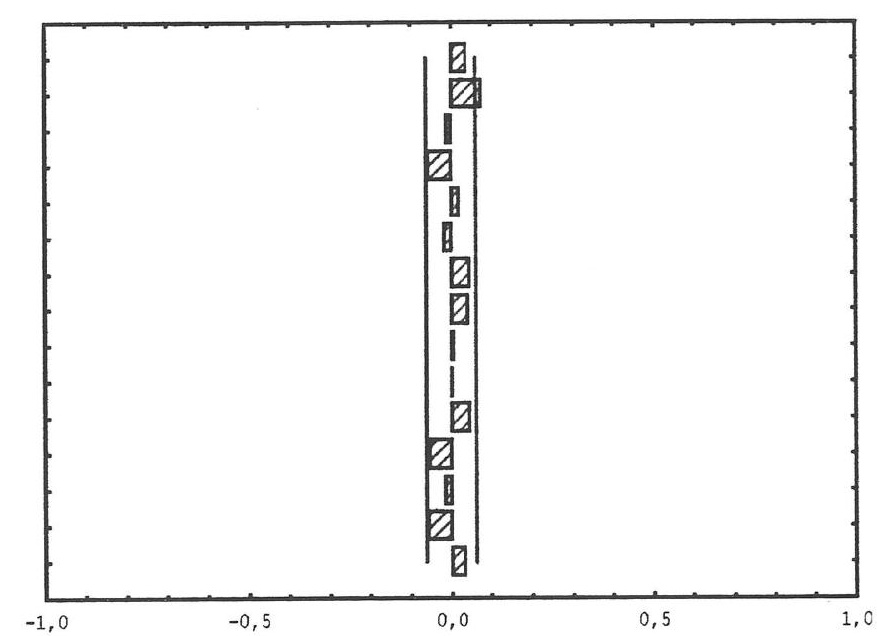

Figura 5.3: Função de autocorrelação de $\lambda$

Nas figuras (5.2) e (5.3), as amostras obtidas pelo algoritmo de Gibbs apresentam funções de autocorrelação próximas a zero, ou seja, são nãocorrelacionadas.

Com a amostra obtida, podemos encontrar os resumos a posteriori e as aproximações das densidades a posteriori marginais para $\mathrm{N}^{\prime}$ e $\lambda$.

\begin{tabular}{|c|c|c|c|c|}
\hline & Média & Mediana & Desvio-padrão & Int. Cred. 95\% \\
\hline $\mathbf{N}^{\prime}$ & 6.074 & 6.0 & 3.38881 & $(1 ; 14)$ \\
$\lambda$ & 0.006823 & 0.00665 & 0.001712 & $(0.004 ; 0.01069)$ \\
\hline
\end{tabular}

Tabela 5.2: Resumos a posteriori para o modelo de JM 


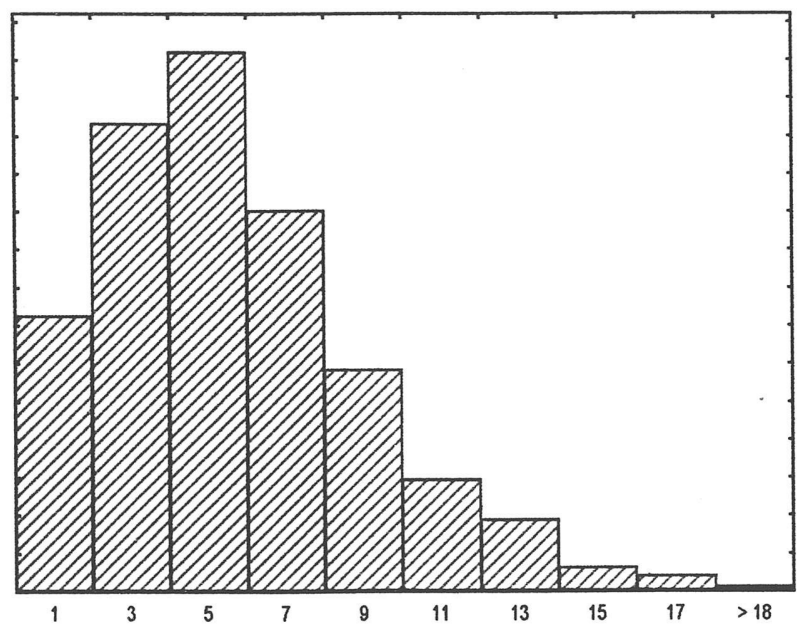

Figura 5.4: Aproximação da densidade a posteriori marginal de $\mathrm{N}^{\prime}=\mathrm{N}-26$.

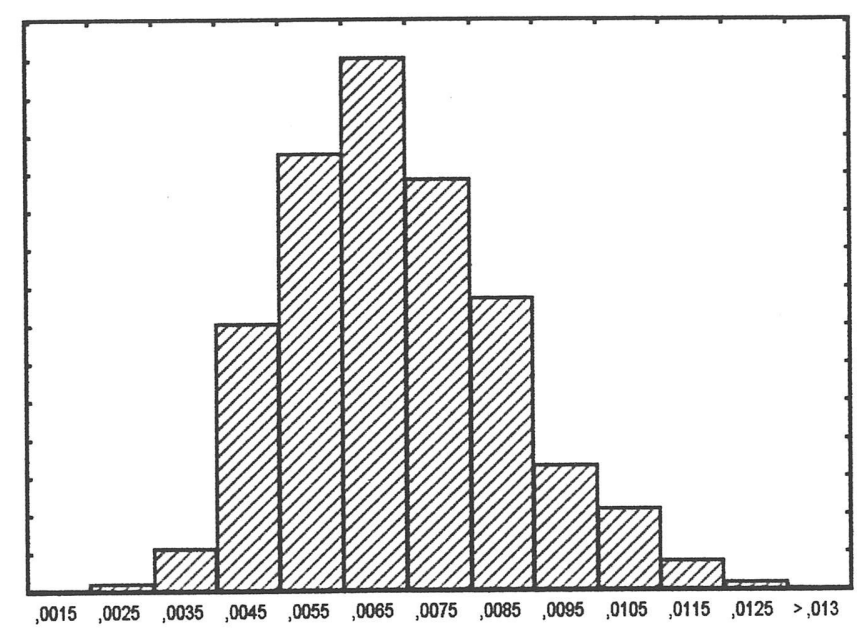

Figura 5.5: Aproximação da densidade a posteriori marginal de $\lambda$.

Comparando os e. m. v. para $\mathrm{N}$ e $\lambda\left(\mathrm{N}^{\prime}=5.21553\right.$ e $\left.\hat{\lambda}=0.00685\right)$, obtidos na análise clássica, com as estimativas pontuais obtidas através da análise Bayesiana (ver tabela 5.2), verificamos uma proximidade nos valores obtidos. Já as estimativas por intervalo (percentil 95\%) mostram-se mais precisas na análise Bayesiana.

Como vimos na seção 3.4.1, uma das formas de verificar a convergência é através da análise gráfica. Nas figuras (5.6) e (5.7) temos as trajetórias das cadeias ao longo das iterações.

Como os gráficos apresentam repetidamente o mesmo comportamento qualitativo e quantitativo, podemos então concluir pela convergência das cadeias para a distribuição de equilíbrio, na qual estamos interessados. 


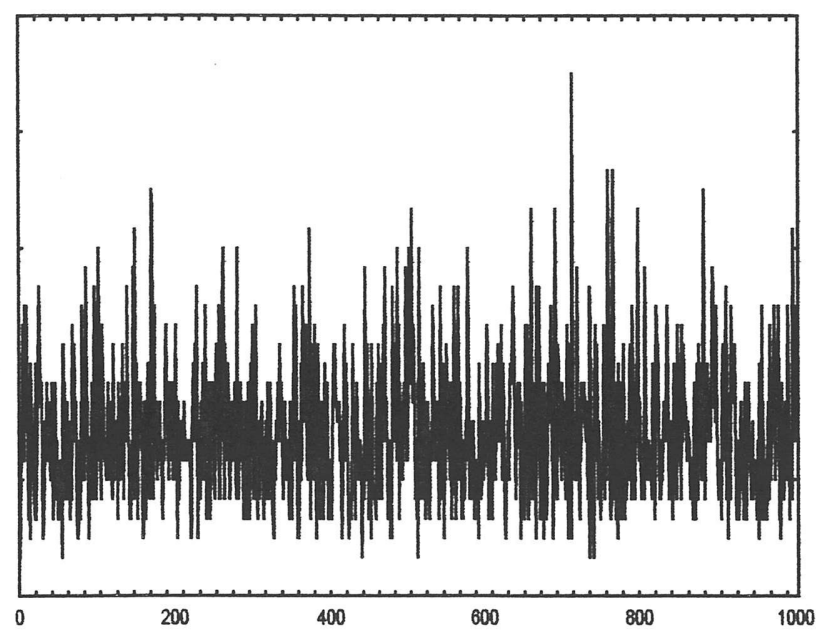

Figura 5.6: Trajetória das cadeias $-\mathrm{N}^{\prime}$.

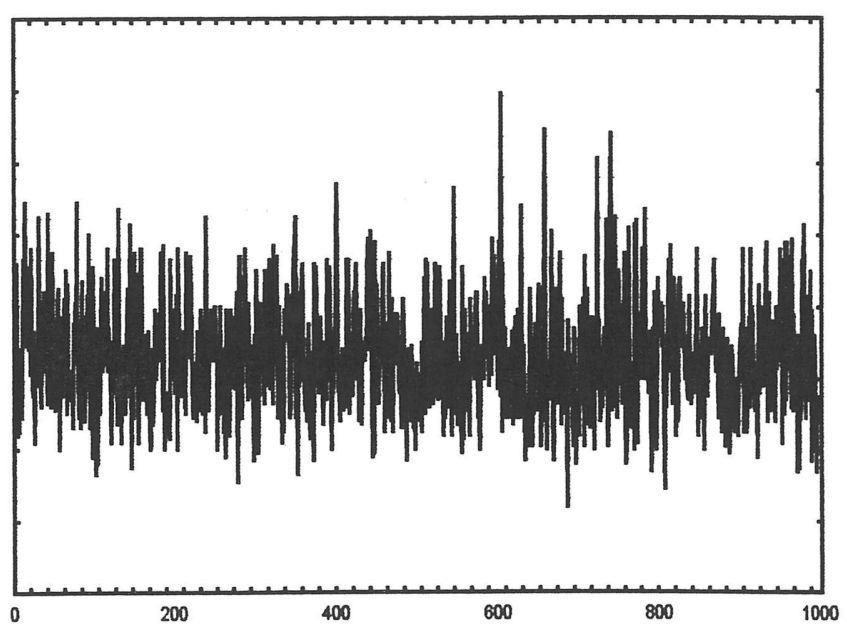

Figura 5.7: Trajetória das cadeias $-\lambda$.

Uma outra forma de verificar a convergência é através do método proposto por Gelman e Rubin (1992), baseado em técnicas de análise de variância. De acordo com esse método (seção 3.4.1), a convergência da cadeia estará assegurada se R for próximo de 1.

Os valores de $\mathrm{R}$ para $\mathrm{N}^{\prime}$ e $\lambda$ obtidos das amostras selecionadas são dados por:

$$
\begin{aligned}
& \hat{\mathrm{R}}_{\mathrm{N}^{\prime}}=1.0081, \\
& \hat{\mathrm{R}}_{\lambda}=1.0028 .
\end{aligned}
$$

Dessa forma, podemos admitir que a convergência foi atingida e que essa amostra, obtida através de simulação, dirige-se para a distribuição de interesse. 


\subsection{Exemplo Considerando o Modelo de Captura-Recaptura de Nayak}

Considerando o mesmo conjunto de dados da tabela (5.1), geramos os contadores $\mathrm{M}_{\mathrm{i}} \approx$ Poisson $\left[\lambda\left(\tau-\mathrm{t}_{(\mathrm{i})}\right)\right]$, onde $\tau=300, \mathrm{r}=26, \lambda=0.00685 \mathrm{e}$ obtivemos $\mathrm{m}=\sum_{\mathrm{i}=1}^{\mathrm{r}} \mathrm{m}_{\mathrm{i}}=32$.

\section{Análise Clássica}

Os estimadores de máxima verossimilhança de $\mathrm{N}$ e $\lambda$, são dados por:

$$
\begin{gathered}
\hat{\mathrm{N}}=29.83, \\
\hat{\lambda}=\frac{\mathrm{r}+\mathrm{m}}{\hat{\mathrm{N}} \tau}=0.00648 .
\end{gathered}
$$

As matrizes de informação observada (2.25) e de variância-covariância assintótica de $\hat{\mathrm{N}}$ e $\hat{\lambda}$ são dadas, respectivamente, por:

$$
\begin{gathered}
I_{0}(N, \lambda)=\left[\begin{array}{cc}
0.19697 & 300 \\
300 & 1381268.1
\end{array}\right], \\
I_{0}^{-1}(N, \lambda)=\left[\begin{array}{cc}
7.58647 & -0.001648 \\
-0.001648 & 0.0000011
\end{array}\right] .
\end{gathered}
$$


Os intervalos de confiança $95 \%$ aproximados para $\mathrm{N}$ e $\lambda$, considerando-se a aproximação Normal assintótica dos e.m.v., são dados por:

$$
\begin{aligned}
& (24.4315<\mathrm{N}<35.2285), \\
& (0.00444<\lambda<0.00852) .
\end{aligned}
$$

A função de verossimilhança profile (2.21) é expressa por

$$
L_{p}(N)=\frac{N(N-1) \ldots(N-r+1)}{N^{m+r}}=\frac{\prod_{i=1}^{26}(N-i+1)}{N^{58}}
$$

O gráfico da função $L_{p}(N)$ é mostrado na figura (5.8). Podemos observar que a função de verossimilhança profile é maximizada no ponto $\hat{\mathrm{N}}_{\mathrm{p}}=30$.

$$
\mathrm{L}_{\mathrm{p}}(\mathrm{N}) * 10^{54}
$$

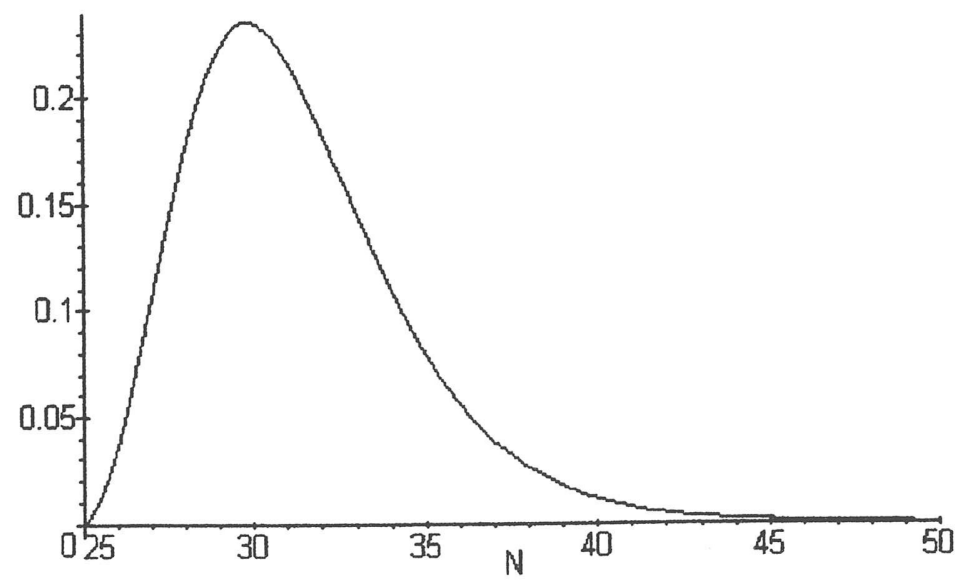

Figura 5.8: Gráfico da profile de N. 


\section{Análise Bayesiana}

Para o modelo de captura-recaptura de Nayak, consideramos priori nãoinformativas, ou seja, supomos que o pesquisador não tem informação prévia a respeito dos parâmetros $\mathrm{N}$ e $\lambda$. Portanto, a densidade a priori conjunta é dada por

$$
\mathrm{p}(\mathrm{N}, \lambda) \propto \frac{1}{\mathrm{~N} \lambda} .
$$

As densidades a posteriori condicionais requeridas para o algoritmo de Gibbs Sampling são indicadas por:

$$
\begin{aligned}
& \lambda \mid \mathrm{N} \sim \Gamma[58,300 \mathrm{~N}], \\
& \pi(\mathrm{N} \mid \lambda) \propto \psi_{1}(\mathrm{~N}, \lambda),
\end{aligned}
$$

onde $\psi_{1}(\mathrm{~N}, \lambda)=\exp \left\{\sum_{\mathrm{i}=2}^{26} \ln (\mathrm{N}-\mathrm{i}+1)-300 \mathrm{~N} \lambda\right\}$.

Para a densidade a posteriori condicional de $\mathrm{N}$, não encontramos um núcleo de transição. Nesse caso, devemos utilizar um núcleo de transição que seja simétrico. Para esse núcleo, escolhemos a distribuição Binomial $\operatorname{com} \mathrm{n}=60 \mathrm{e} \mathrm{p}=0.5$. $\mathrm{O}$ valor de $\mathrm{n}$ foi escolhido de forma que a média da distribuição seja próxima ao estimador de máxima verossimilhança de $\mathrm{N}(\mathrm{E}(\mathrm{X})=\mathrm{np}=30)$. 
Com as distribuições condicionais obtidas, geramos cinco cadeias com 2000 iterações cada, considerando, segundo uma análise preliminar dos dados e os valores dos estimadores de máxima verossimilhança para $\mathrm{N}$ e $\lambda$, diferentes valores iniciais para as cadeias.

Para cada parâmetro, desprezamos as 1000 iterações iniciais em cada cadeia e dos valores restantes, selecionamos a iteração $1005,1010, \ldots, 1095,2000$, que de cinco cadeias produziu uma amostra de tamanho 1000 .

Nas figuras (5.90 e (5.10) temos as trajetórias das amostras de $\mathrm{N}$ e $\lambda$., obtidas pelo algoritmo de Gibbs com Metropolis.

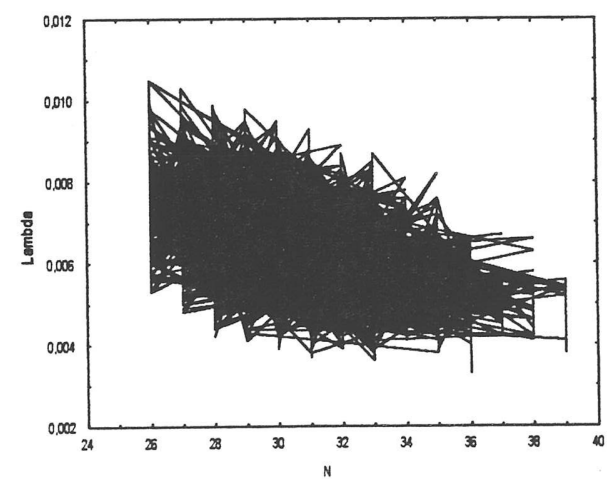

Figura 5.9: Trajetória das amostras não selecionadas de $\mathrm{N}$ e $\lambda$.

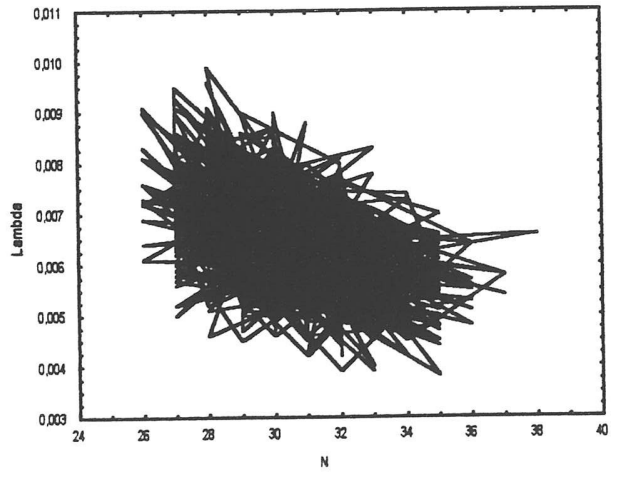

Figura 5.10: Trajetórias das amostras selecionadas de $\mathrm{N}$ e $\lambda$.

Percebemos pelas figuras acima que as amostras selecionadas convergem para uma distribuição de equilíbrio. 
Essa seleção foi realizada de forma a garantir uma baixa correlação entre os valores obtidos na amostra. Isso pode ser confirmado através das figuras (5.11) e (5.12), que ilustram as funções de autocorrelação das amostras geradas para $\mathrm{N}$ e $\lambda$.
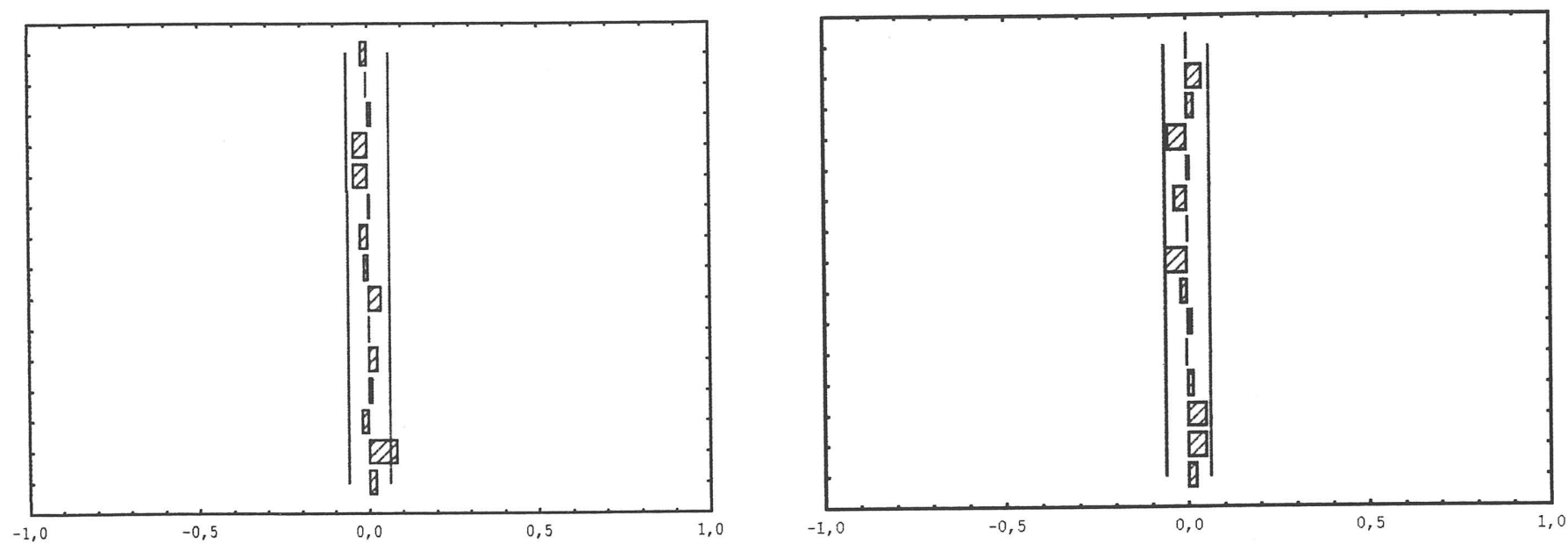

Figura 5.11: Função de autocorrelação de N. Figura 5.12: Função de autocorrelação de $\lambda$

Considerando as amostras não-correlacionadas, geradas pelo algoritmo de Gibbs com Metropolis, podemos obter os resumos a posteriori para os parâmetros $\mathrm{N}$ e $\lambda$ (tabela 5.3) e também uma aproximação das densidades a posteriori marginais dos parâmetros (figuras 5.13 e 5.14).

\begin{tabular}{|c|c|c|c|c|}
\hline & Média & Mediana & Desvio-padrão & Int. Cred. 95\% \\
\hline $\mathbf{N}$ & 30.363 & 30 & 2.19592 & $(27 ; 35)$ \\
$\lambda$ & 0.0064 & 0.0063 & 0.00096 & $(0.0046 ; 0.0085)$ \\
\hline
\end{tabular}

Tabela 5.3: Resumos a posteriori para o modelo de Captura-Recaptura de Nayak. 


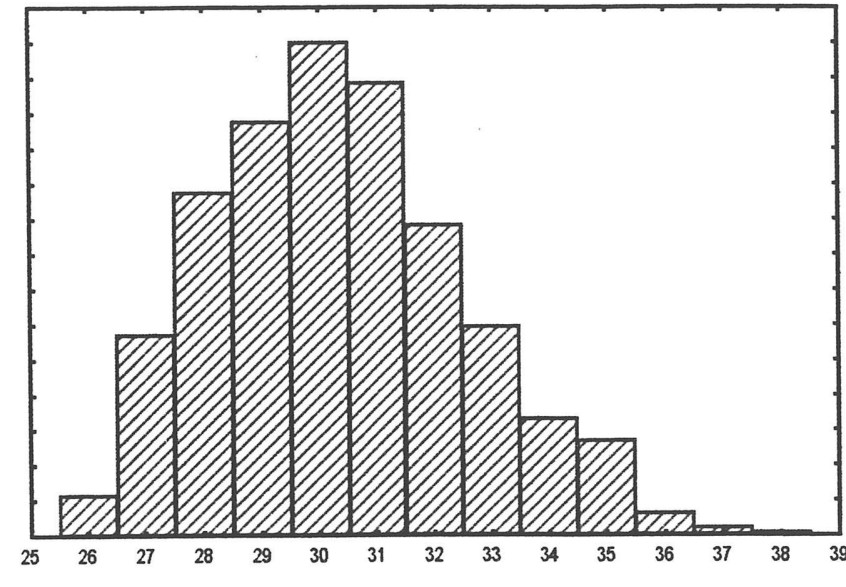

Figura 5.13: Aproximação da densidade a posteriori marginal de $\mathrm{N}$.

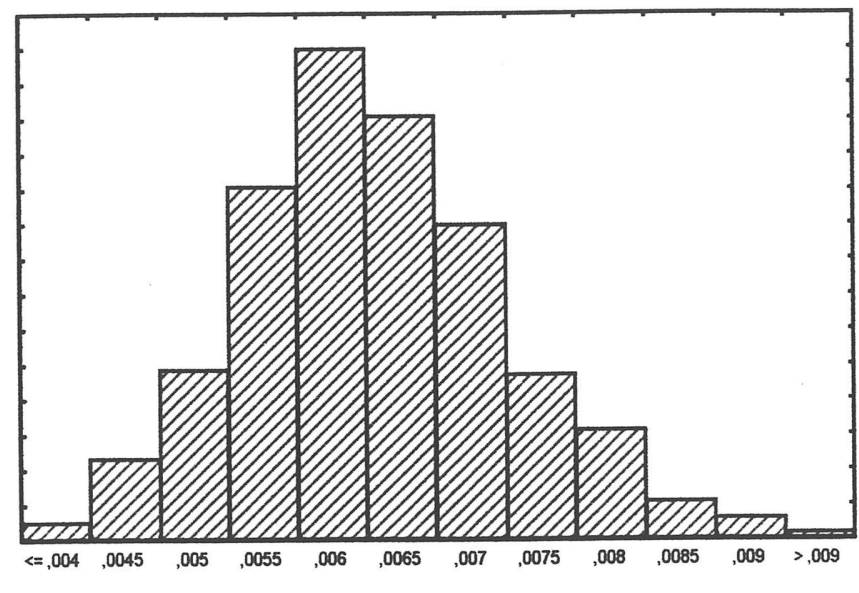

Figura 5.14: Aproximação da densidade a posteriori marginal de $\lambda$.

Podemos perceber que os estimadores de máxima verossimilhança para $\mathrm{N}$ e $\lambda$ obtidos na análise clássica, são muito próximos às estimativas pontuais, obtidas através das amostras geradas pelo algoritmo de Gibbs com Metropolis.

Os intervalos de credibilidade para $\mathrm{N}$ e $\lambda(95 \%)$ são mais precisos do que os intervalos de confiança (95\%) obtidos segundo a teoria assintótica dos e.m.v..

Os valores de $\mathrm{R}$ (fator de redução de escala), descritos por Gelman e Rubin (1992), obtidos das amostras selecionadas para $\mathrm{N}$ e $\lambda$, são dados por:

$$
\begin{aligned}
& \hat{\mathrm{R}}_{\mathrm{N}}=1.0053, \\
& \hat{\mathrm{R}}_{\lambda}=1.0092 .
\end{aligned}
$$


Como os valores são próximos de 1 , podemos admitir que a convergência ocorreu e que essa amostra, obtida através de simulação, converge para a distribuição de interesse.

Podemos encontrar a moda a posteriori $\left(\hat{\mathrm{N}}_{0}\right)$ de $\mathrm{N}$ utilizando a expressão (3.19), ou seja, $\hat{\mathrm{N}}_{0}=\mathrm{k}$ se, e somente $\mathrm{se}, \mathrm{h}_{\mathrm{r}, \mathrm{k}+1}^{* *}<\mathrm{m}<\mathrm{h}_{\mathrm{r}, \mathrm{k}}^{* *}$

Como $\mathrm{m}=32$ pertence ao intervalo $\left[\mathrm{h}_{26,31} ; \mathrm{h}_{26,30}\right]=[28.6437 ; 32.4339]$, temos que $\hat{\mathrm{N}}_{0}=30$.

\subsection{Exemplo Considerando o Modelo de Goel e Okumoto}

\section{Análise Clássica}

$\mathrm{Na}$ análise clássica, precisamos usar algum método iterativo para encontrarmos os estimadores de máxima verossimilhança de $\mathrm{N}, \lambda$ e p. Utilizamos o método de Newton-Raphson (ver apêndice 1) e encontramos $\mathrm{p}=1.18$, o que não poderia ter ocorrido, pois $0<\mathrm{p}<1$.

Para resolvermos esse problema, fizemos uma reparametrização, ou seja, consideramos $\eta=\ln [(1-p) / p]$. Agora, para qualquer valor de $\eta$, teremos $0<\mathrm{p}<1$, pois $\mathrm{p}=\{1 /[\exp (\eta)+1]\}$. 
$\mathrm{O}$ valor obtido para $\mathrm{p}$ foi $\mathrm{p}=0.754$, pois $\eta=-1.12$. Portanto, os e.m.v. são dados por:

$$
\hat{N}=28.43, \quad \hat{\lambda}=0.00608 \quad \text { e } \quad \hat{p}=0.754
$$

\section{Análise Bayesiana}

Considerando-se o modelo de Goel e Okumoto (2.44) e o conjunto de dados da tabela (5.1), assuminos a densidade a priori conjunta (3.22) para $\mathrm{N}, \lambda$ e $p$, onde $a_{1}=b_{1}=2.5$ são constantes conhecidas, obtidas através de uma análise preliminar dos dados.

De (3.24), as densidades a posteriori condicionais de $\mathrm{N}, \lambda$ e $p$, requeridas para o algoritmo de Gibbs Sampling, são dadas por:

$$
\begin{gathered}
\lambda \mid \mathrm{N}, p \sim \Gamma[26,2492 p+(\mathrm{N}-26 p) 250], \\
\pi(\mathrm{N} \mid \lambda, p) \propto \psi_{2}(\mathrm{~N}, \lambda, p),
\end{gathered}
$$

onde $\psi_{2}(\mathrm{~N}, \lambda, p)=\exp \left\{\sum_{\mathrm{i}=1}^{\mathrm{r}} \ln [\mathrm{N}-\mathrm{p}(\mathrm{i}-1)]-\ln (\mathrm{N})-250 \lambda(\mathrm{N}-26 \mathrm{p})\right\} \mathrm{e}$

$$
\pi(p \mid \mathrm{N}, \lambda) \propto p^{1.5}(1-p)^{1.5} \psi_{3}(\mathrm{~N}, \lambda, p),
$$


onde $\psi_{3}(\mathrm{~N}, \lambda, p)=\exp \left\{\sum_{\mathrm{i}=1}^{\mathrm{r}} \ln [\mathrm{N}-\mathrm{p}(\mathrm{i}-1)]-\lambda[2492 \mathrm{p}+250(\mathrm{~N}-26 \mathrm{p})]\right\}$.

Considerando-se as densidades condicionais acima, geramos cinco cadeias com 2000 iterações cada, utilizando o algoritmo Gibbs Sampling com MetropolisHastings. Foram considerados diferentes valores iniciais para as cadeiaa, baseados numa análise preliminar dos dados.

Para cada parâmetro, selecionamos a iteração $1005,1010, \ldots, 1095,2000$, que de cinco cadeias produziu uma amostra de tamanho 1000 .

A seleção dessa amostra foi feita de forma a garantir independência e uma pequena autocorrelação dos valores obtidos, que pode ser visualizada através das figuras (5.15), (5.16) e (5.17).
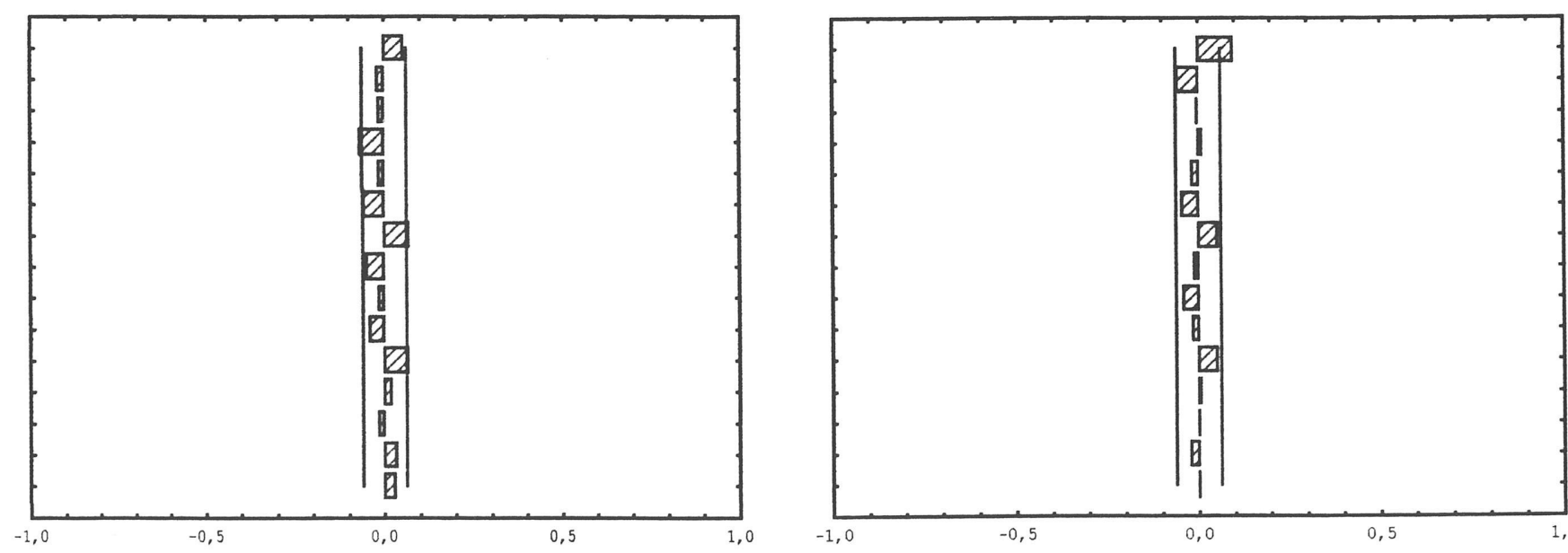

Figura 5.15: Função de autocorrelação de N. Figura 5.16: Função de autocorrelação de $\lambda$. 


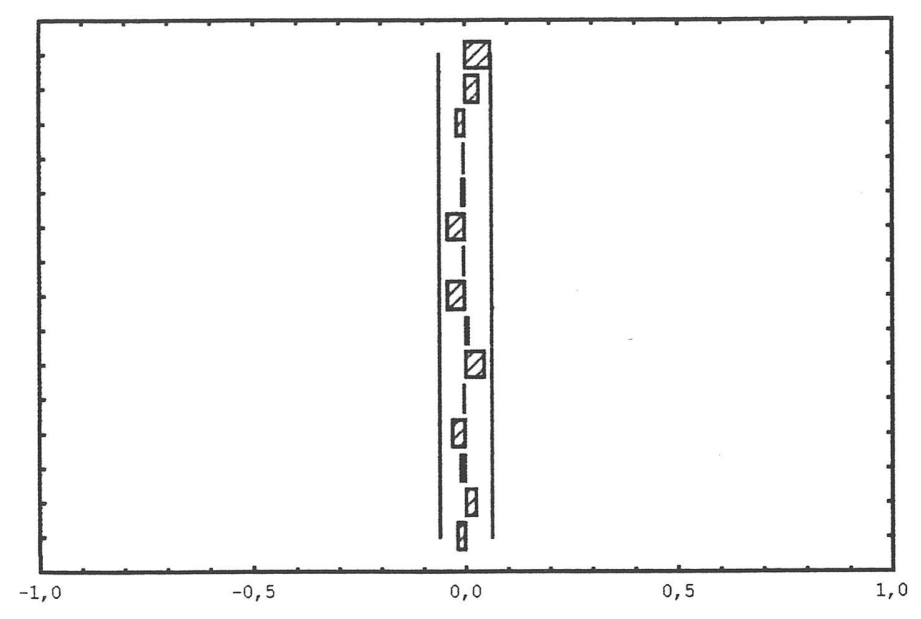

Figura 5.17: Função de autocorrelação de $p$.

Podemos observar, através das figuras (5.15), (5.16) e (5.17), que as amostras não são correlacionadas, devido a maioria dos termos apresentarem-se próximos a zero.

A tabela (5.4) mostra os resumos a posteriori para os parâmetros $\mathrm{N}, \lambda$ e $p$ e as figuras (5.18), (5.19) e (5.20) mostram as aproximações das densidades a posteriori marginais para $\mathrm{N}, \lambda$ e $p$.

\begin{tabular}{|c|c|c|c|c|}
\hline & Média & Mediana & Desvio-padrão & Int. Cred. 95\% \\
\hline $\mathbf{N}$ & 30.558 & 30 & 3.06497 & $(26 ; 37)$ \\
$\lambda$ & 0.00505 & 0.0049 & 0.001467 & $(0.0028 ; 0.0088)$ \\
$\mathbf{p}$ & 0.56712 & 0.5828 & 0.19404 & $(0.1593 ; 0.8947)$ \\
\hline
\end{tabular}

Tabela 5.4: Resumos a posteriori para o modelo de Goel e Okumoto 


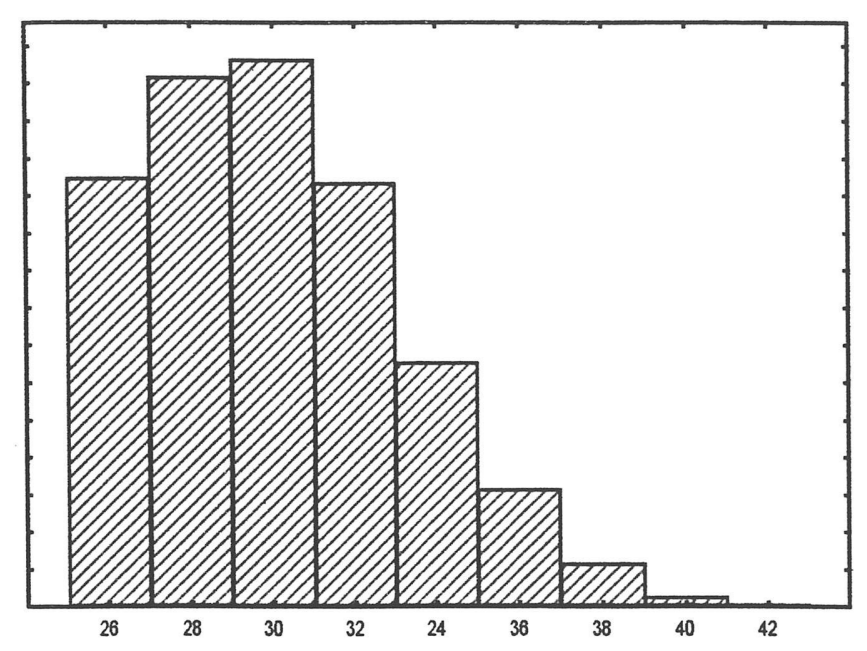

Figura 5.18: Aproximação da densidade a posteriori marginal de $\mathrm{N}$.

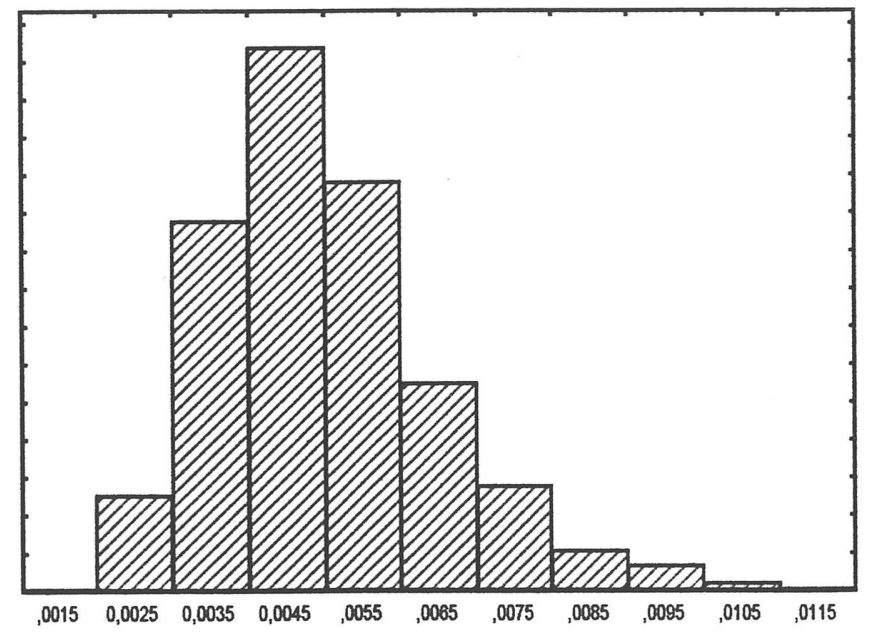

Figura 5.19: Aproximação da densidade a posteriori marginal de $\lambda$.

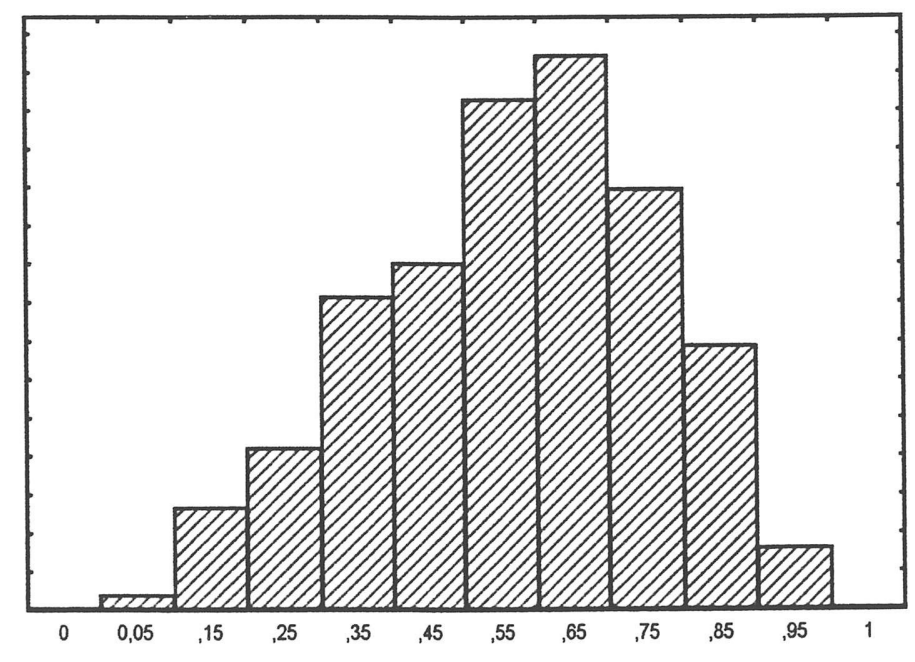

Figura 5.20: Aproximação da densidade a posteriori marginal de $p$.

Podemos perceber que os estimadores de máxima verossimilhança de $\lambda$ e $p$ obtidos na análise clássica, são maiores que as estimativas pontuais obtidas através das amostras geradas pelo algoritmo de Gibbs com Metropolis. O e.m.v. de N é menor na análise clássica, devido ao fato de $\mathrm{p}$ ser maior. 
Os fatores de redução de escala para $\mathrm{N}, \lambda$ e $p$ são dados por:

$$
\hat{\mathrm{R}}_{\mathrm{N}}=1.0031, \quad \hat{\mathrm{R}}_{\lambda}=1.0013, \quad \hat{\mathrm{R}}_{\mathrm{p}}=1.0019 .
$$

Avaliando-se a proximidade dos fatores $\hat{\mathrm{R}}_{\mathrm{N}}, \hat{\mathrm{R}}_{\lambda}$ e $\hat{\mathrm{R}}_{\mathrm{p}}$ a 1 , podemos concluir pela convergência das amostras para uma distribuição de equilíbrio.

\subsection{Exemplo Considerando o Modelo de Goel e Okumoto com Captura- Recaptura}

\section{$1^{\circ}$ caso: $p$ conhecido}

Considerando o modelo de Goel e Okumoto com captura-recaptura (2.57) e o conjunto de dados da tabela (5.1), assuminos a densidade a priori conjunta (3.31) para $\mathrm{N}$ e $\lambda(p=0.7$ é assumido ser conhecido).

De (3..37), as densidades a posteriori condicionais de $\mathrm{N}$ e $\lambda$, requeridas para $\mathrm{o}$ algoritmo de Gibbs são dadas por:

$$
\begin{aligned}
& \lambda \mid \mathrm{N} \sim \Gamma[58,300 \mathrm{~N}+1592.4], \\
& \pi(\mathrm{N} \mid \lambda) \propto \psi_{4}(\mathrm{~N}, \lambda),
\end{aligned}
$$


onde $\psi_{4}(\mathrm{~N}, \lambda)=\exp \left\{\sum_{\mathrm{i}=1}^{\mathrm{r}-1} \ln [\mathrm{N}-0.7 \mathrm{i}]-300 \mathrm{~N} \lambda\right\}$.

Com as densidades a posteriori condicionais obtidas, obtivemos uma amostra de tamanho 1000 utilizando o algoritmo de Gibbs com Metropolis. Podemos observar através das figuras (5.21) e (5.22) que as amostras selecionadas são não correlacionadas.
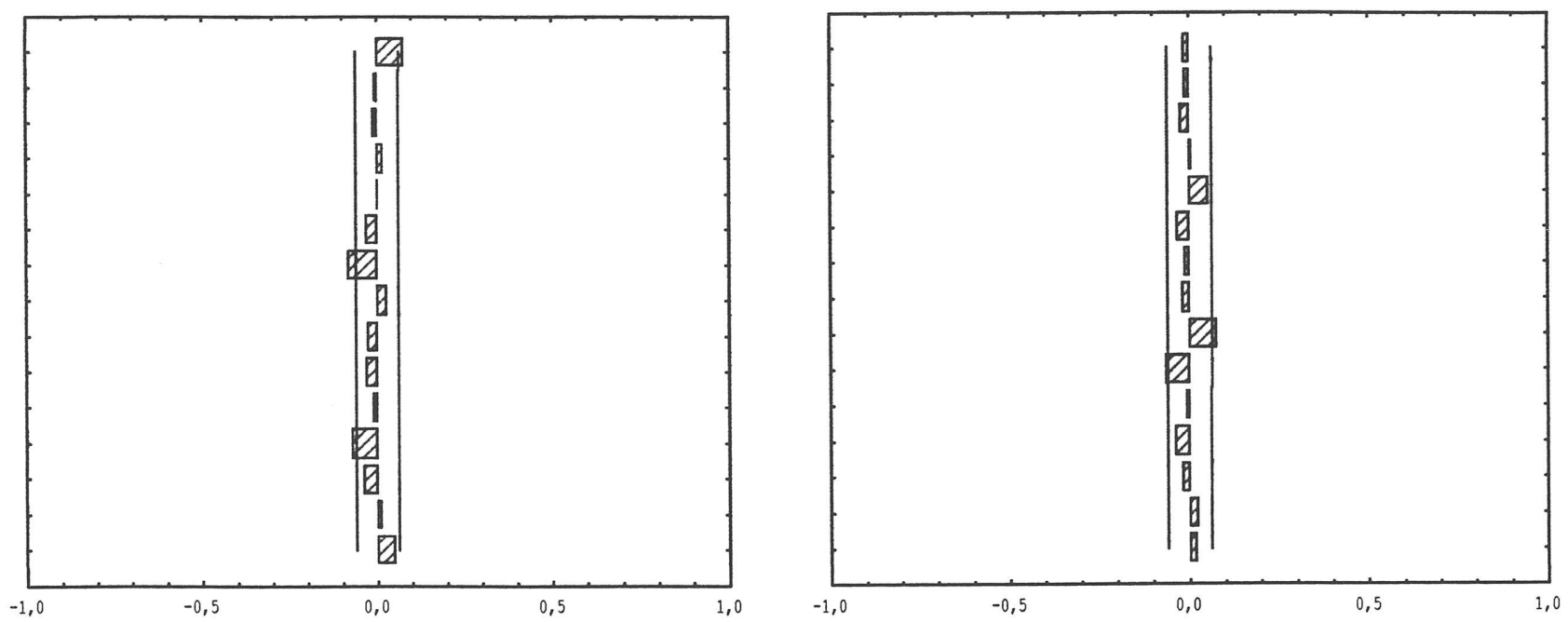

Figura 5.21: Função de autocorrelação de N. Figura 5.22: Função de autocorrelação de $\lambda$.

A tabela (5.5) mostra os resumos a posteriori para os parâmetros $\mathrm{N}$ e $\lambda$ e as figuras (5.23) e (5.24) mostram as aproximações das densidades a posteriori marginais para $\mathrm{N}$ e $\lambda$.

\begin{tabular}{|c|c|c|c|c|}
\hline & Média & Mediana & Desvio-padrão & Int. Cred. 95\% \\
\hline $\mathbf{N}$ & 28.915 & 29.0 & 2.33178 & $(26 ; 34)$ \\
$\lambda$ & 0.00566 & 0.0056 & 0.00795 & $(0.0043 ; 0.0073)$ \\
\hline
\end{tabular}

Tabela 5.5: Resumos a posteriori para o modelo de Goel e Okumoto com captura-recaptura. 


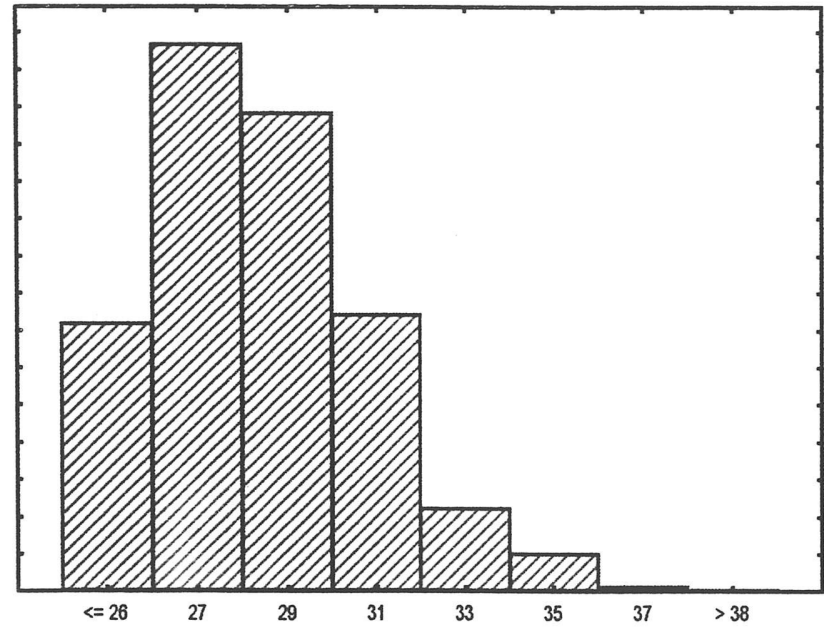

Figura 5.23: Aproximação da densidade a posteriori marginal de $\mathrm{N}$.

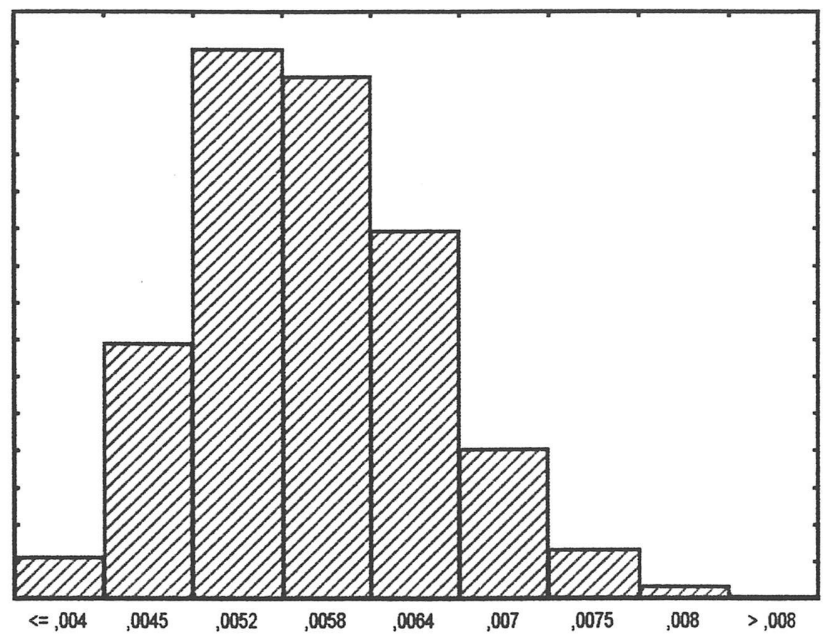

Figura 5.24: Aproximação da densidade a posteriori marginal de $\lambda$.

A convergência das amostras geradas pelo algoritmo de Gibbs Sampling com Metropolis-Hastings pode ser monitorada pelo critério de Gelman e Rubin (1992). Os fatores de redução de escala para $\mathrm{N}$ e $\lambda$ são dados por:

$$
\begin{aligned}
& \hat{\mathrm{R}}_{\mathrm{N}}=1.0007, \\
& \hat{\mathrm{R}}_{\lambda}=0.9988 .
\end{aligned}
$$

Como os fatores $\hat{\mathrm{R}}_{\mathrm{N}}, \hat{\mathrm{R}}_{\lambda}$ são próximos de 1 , podemos concluir pela convergência das amostras à uma distribuição de equilíbrio.

Também podemos verificar a convergência através das figuras (5.25) e (5.26), que mostram as trajetórias das cadeias ao longo das iterações. 


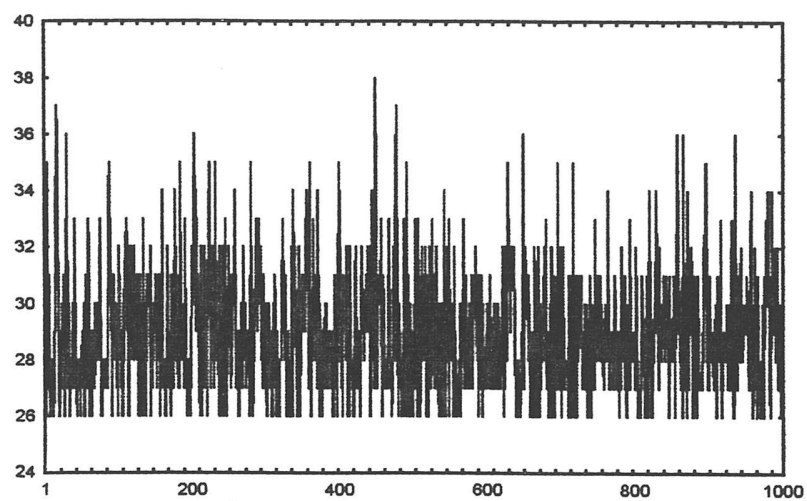

Figura 5.25: Trajetória daas cadeias - N.

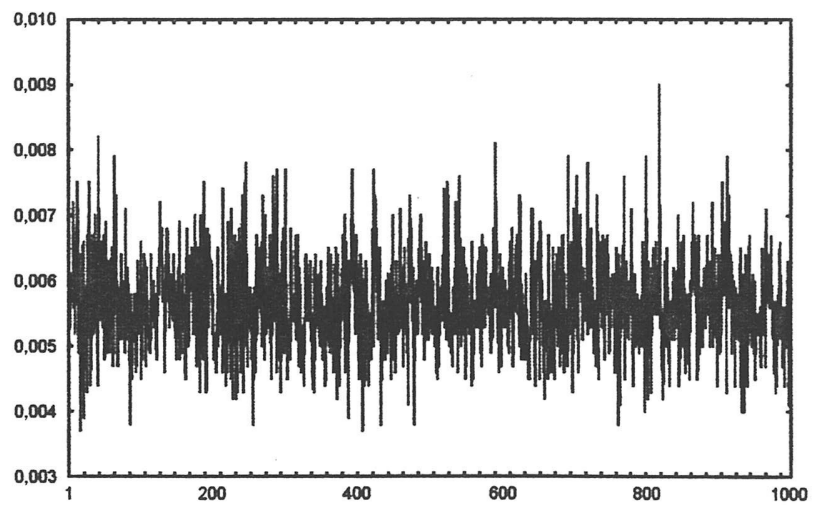

Figura 5.26: Trajetória das cadeias $-\lambda$.

Podemos observar, através das figuras acima, que a convergência ocorreu, devido o comportamento repetitivo das cadeias após um período inicial.

\section{$2^{\circ}$ caso: $p$ desconhecido}

Considerando-se os dados da tabela (5.1) e assumindo agora $p$ desconhecido, a densidade a priori conjunta para os parâmetros $\mathrm{N}, \lambda$ e $p$ é dada, de (3.38), por

$$
\mathrm{p}(\mathrm{N}, \lambda, p) \propto \frac{\gamma_{1}^{\mathrm{N}} \exp \left\{-\gamma_{1}\right\}}{\mathrm{N} !} \lambda^{\mathrm{a}_{4}-1} \exp \left\{-\lambda \mathrm{b}_{4}\right\} \mathrm{p}^{\mathrm{a}_{5}-1}(1-\mathrm{p})^{\mathrm{b}_{5}-1},
$$

onde $\mathrm{a}_{4}=5.49, \mathrm{~b}_{4}=802.11, \gamma_{1}=31, \mathrm{a}_{5}=2.5, \mathrm{~b}_{5}=2.5$ são constantes conhecidas através de uma análise preliminar dos dados.

De (3.40), as densidades a posteriori condicionais requeridas para o algoritmo de Gibbs com Metropolis são expressas por: 


$$
\begin{gathered}
\lambda \mid \mathrm{N}, p \sim \Gamma[63.49,802.11+\mathrm{N} \tau+5308(1-\mathrm{p})], \\
\pi(\mathrm{N} \mid \lambda, p) \propto \frac{31^{\mathrm{N}} \exp \{-31\}}{\mathrm{N} !} \psi_{5}(\mathrm{~N}, \lambda, p),
\end{gathered}
$$

onde $\psi_{5}(\mathrm{~N}, \lambda, p)=\exp \left\{\sum_{\mathrm{i}=1}^{26} \ln [\mathrm{N}-\mathrm{p}(\mathrm{i}-1)]-300 \mathrm{~N} \lambda\right\} \mathrm{e}$

$$
\pi(p \mid \mathrm{N}, \lambda) \propto \mathrm{p}^{1.5}(1-\mathrm{p})^{1.5} \psi_{6}(\mathrm{~N}, \lambda, p),
$$

onde $\psi_{6}(\mathrm{~N}, \lambda, p)=\exp \left\{\sum_{\mathrm{i}=1}^{26} \ln [\mathrm{N}-\mathrm{p}(\mathrm{i}-1)]+5308 \lambda \mathrm{p}\right\}$.

Das cinco cadeias geradas pelo algoritmo de Gibbs com Metropolis, selecionamos, para cada parâmetro, uma amostra de tamanho 1000, de forma a garantir independência e uma baixa correlação dos dados, como pode ser visto nas figuras (5.27), (5.28) e (5.29).
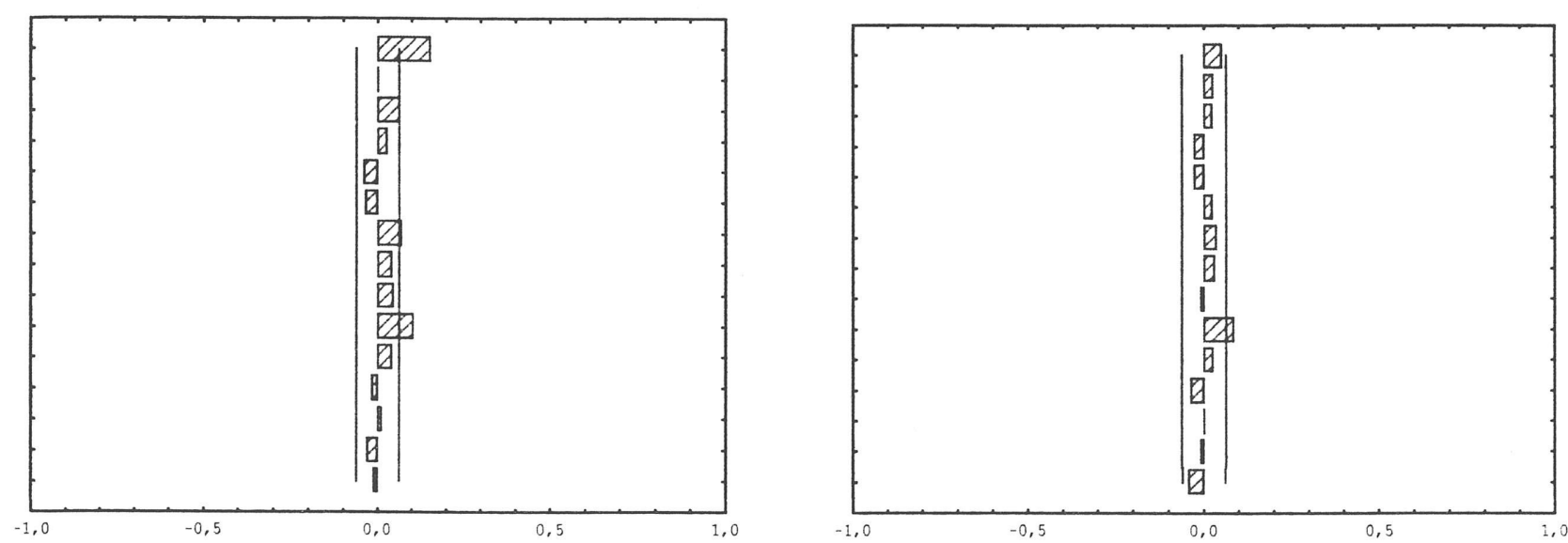

Figura 5.27: Função de autocorrelação de N. Figura 5.28: Função de autocorrelação de $\lambda$. 


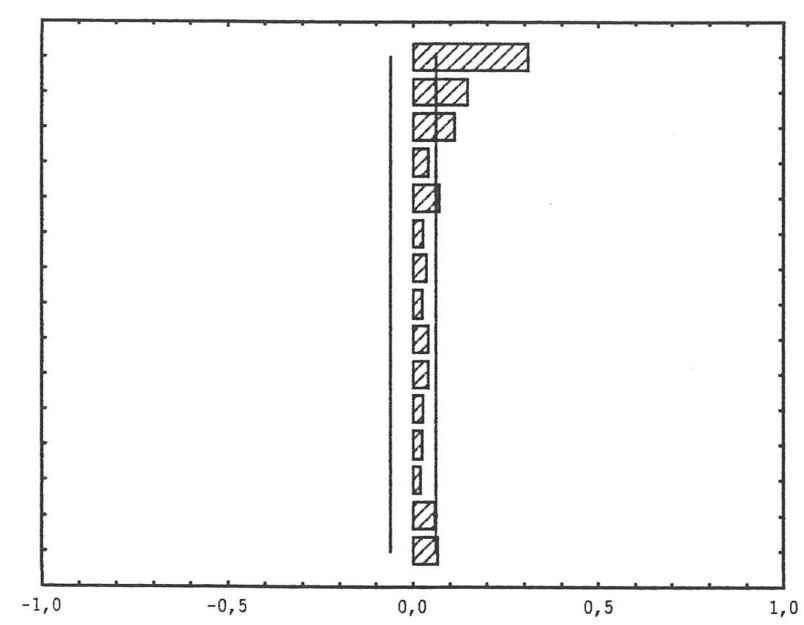

Figura 5.29: Função de autocorrelação de p.

$\mathrm{Na}$ tabela (5.6) temos os resumos a posteriori para os parâmetros $\mathrm{N}, \lambda$ e $p$ do modelo de Goel e Okumoto com captura-recaptura ( $\mathrm{p}$ desconhecido) e nas figuras (5.30), (5.31) e (5.32) mostramos as aproximações das densidades a posteriori marginais para $\mathrm{N}, \lambda$ e $p$ obtidas através do algoritmo de Gibbs Sampling com Metropolis-Hastings.

\begin{tabular}{|c|c|c|c|c|}
\hline & Média & Mediana & Desvio-padrão & Int. Cred. 95\% \\
\hline $\mathbf{N}$ & 29.289 & 29.0 & 2.59928 & $(26 ; 35)$ \\
$\lambda$ & 0.00581 & 0.0058 & 0.00877 & $(0.0042 ; 0.0076)$ \\
$\mathbf{p}$ & 0.7474 & 0.7658 & 0.13211 & $(0.4148 ; 0.9597)$ \\
\hline
\end{tabular}

Tabela 5.6: Resumos a posteriori para o modelo de Goel e Okumoto com captura-recaptura. 


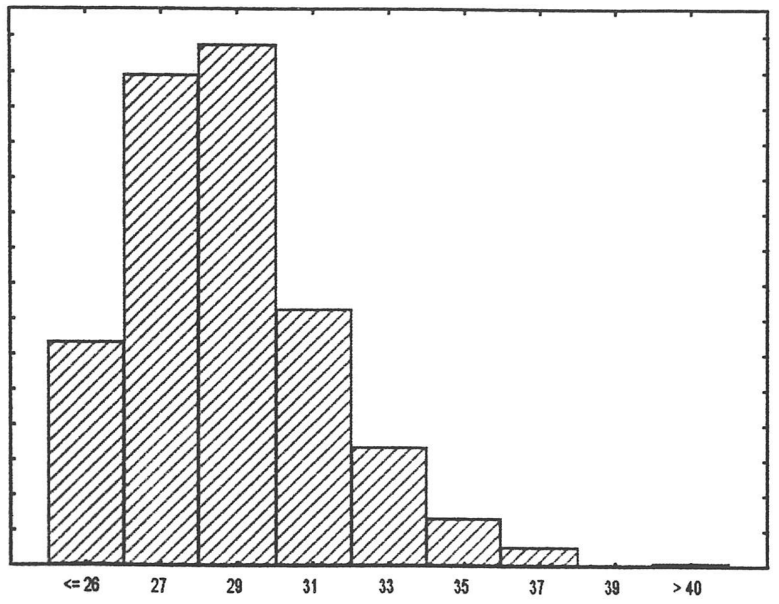

Figura 5.30: Aproximação da densidade a posteriori marginal de $\mathrm{N}$.

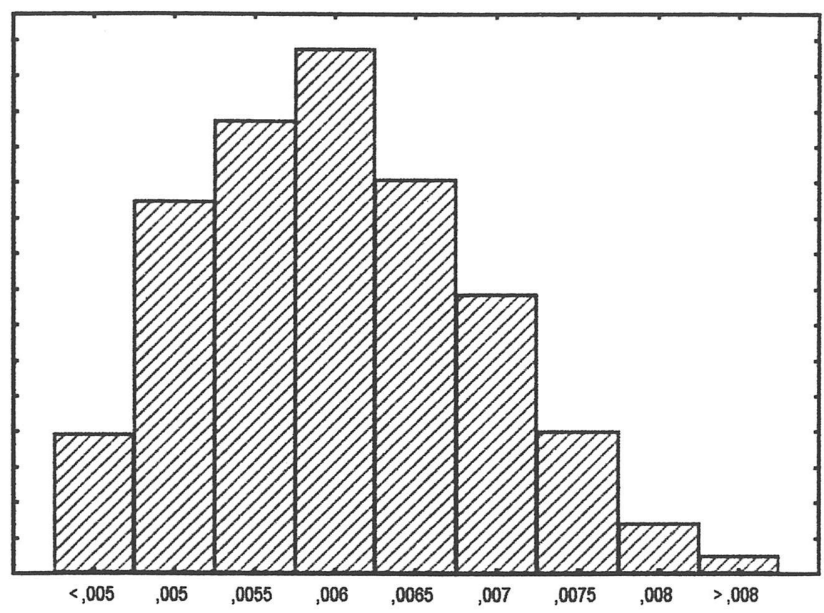

Figura 5.31: Aproximação da densidade a posteriori marginal de $\lambda$.

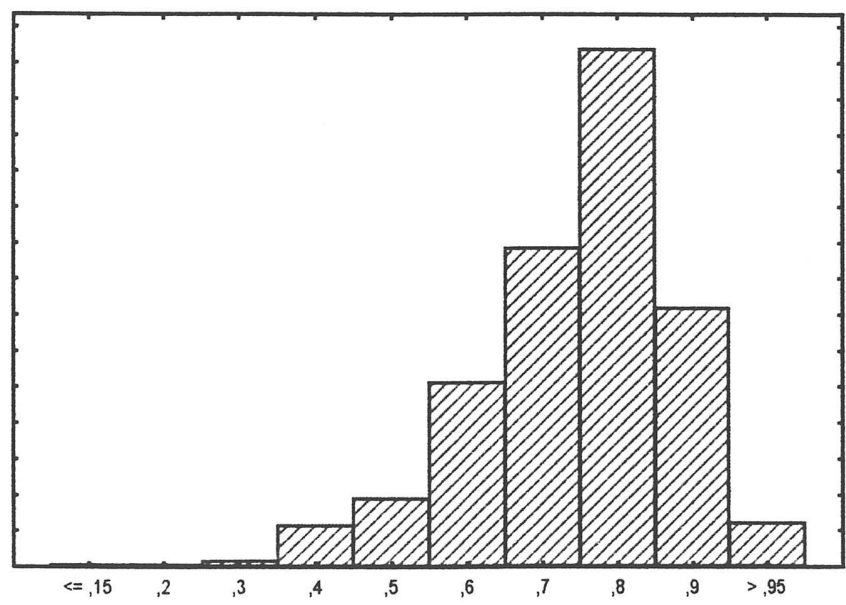

Figura 5.32: Aproximação da densidade a posteriori marginal de $p$.

Os valores de redução de escala obtidos para a verificação da convergência são dados por:

$$
\hat{\mathrm{R}}_{\mathrm{N}}=1.0019, \quad \hat{\mathrm{R}}_{\lambda}=1.0006, \quad \hat{\mathrm{R}}_{\mathrm{p}}=1.0079 .
$$


Como os fatores $\hat{R}_{N}, \hat{R}_{\lambda}$ e $\hat{R}_{p}$ são próximos de 1 , podemos concluir pela convergência das amostras à uma distribuição de equilíbrio.

Podemos também verificar a ocorrência da convergência pelas figuras (5.33), (5.34) e (5.35), devido ao comportamento repetitivo das cadeias ao longo das iterações.
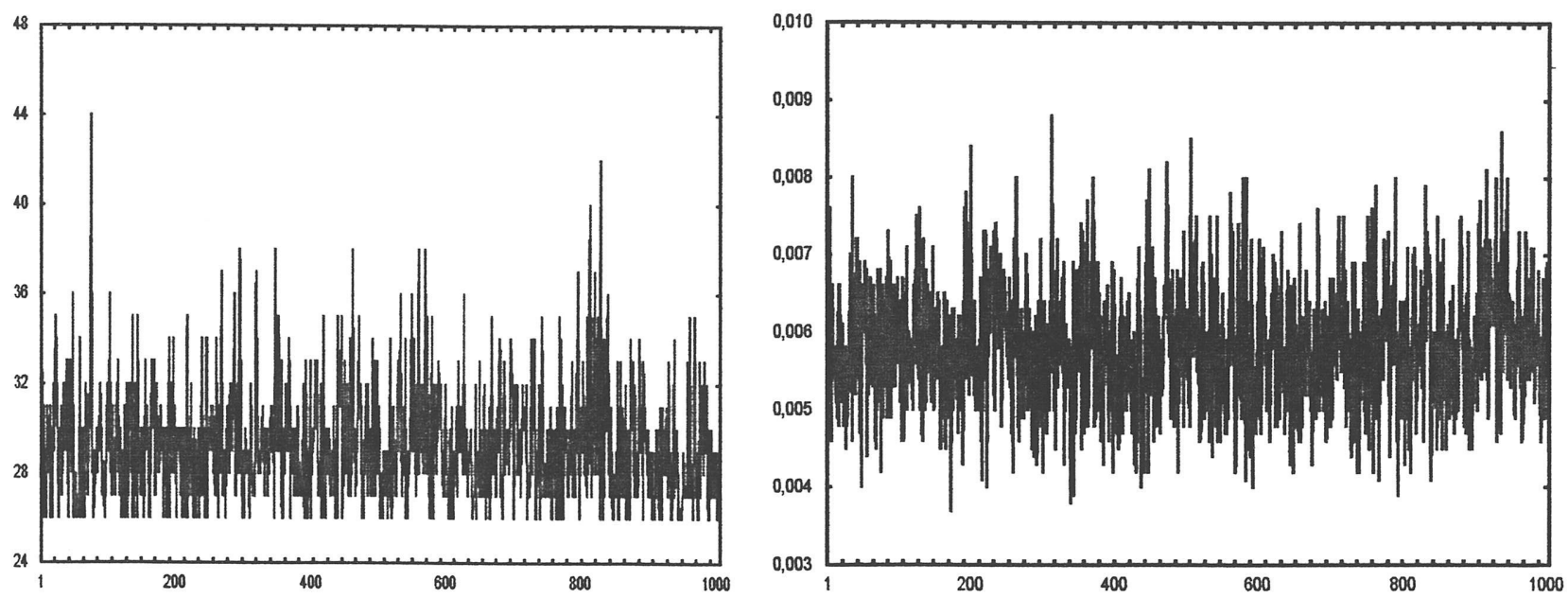

Figura 5.33: Trajetória das cadeias - $N$. Figura 5.34: Trajetória das cadeias $-\lambda$.

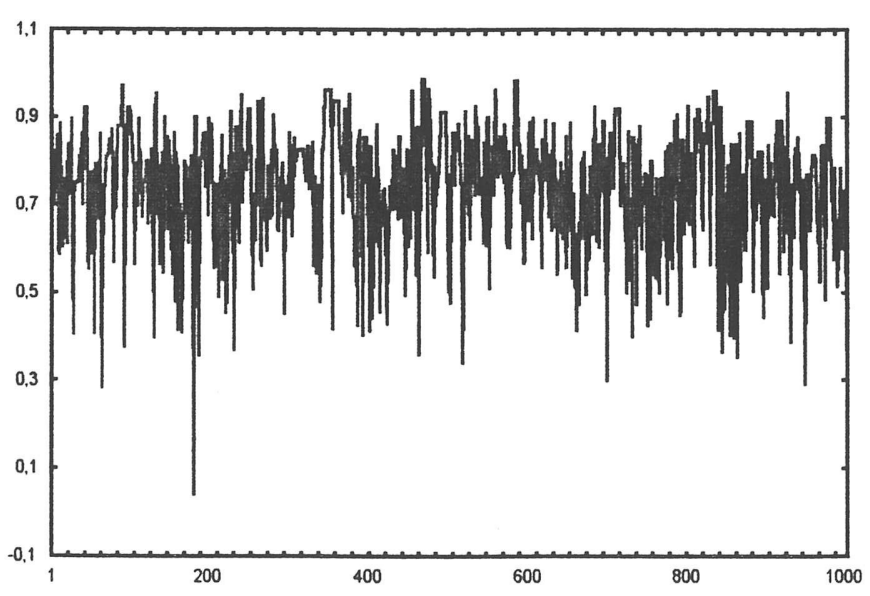

Figura 5.35: Trajetória das cadeias - $p$. 


\subsection{Exemplos Utilizando as Medidas de Divergência}

Agora iremos gerar dados da distribuição em questão (modelo de Goel e Okumoto com captura-recaptura, (2.57)):

$$
\begin{aligned}
& \mathrm{W}_{\mathrm{i}} \mid \mathrm{N}, \lambda \approx \operatorname{Exp}\{\lambda[\mathrm{N}-\mathrm{p}(\mathrm{i}-1)]\}, \mathrm{i}=1, \ldots, \mathrm{r}+1, \\
& \mathrm{M}_{\mathrm{i}} \approx \operatorname{Poisson}\left[\lambda\left(\tau-\mathrm{T}_{(\mathrm{i})}\right)\right], \mathrm{i}=1, \ldots, \mathrm{r},
\end{aligned}
$$

e observar o comportamento das medidas de divergência (distâncias de KullbackLeibler e Variação Total).

Supomos $\lambda=1, N=30,40$ e 50 conhecidos, e diferentes valores de r. Os tempos entre falhas $\left(\mathrm{w}_{\mathrm{i}}\right)$ e os contadores $\left(\mathrm{M}_{\mathrm{i}}\right)$ foram gerados no pacote estatístico "Minitab". Com os dados obtidos, calculamos as distâncias para três diferentes valores de $r$, sempre fixando a posteriori marginal exata de $N(3.33)$ com $p=1$ e comparando com os valores de $\mathrm{p}=0.10,0.30,0.50$ e 0.80 .

Os gráficos das distâncias são mostrados a seguir. Comparando as figuras (5.38) com (5.40) e (5.39) com (5.41), percebemos que, com o aumento de $\mathrm{N}$ de 40 para 50, as distâncias de KL e VT para r $=20$ e 30, diminuem. 


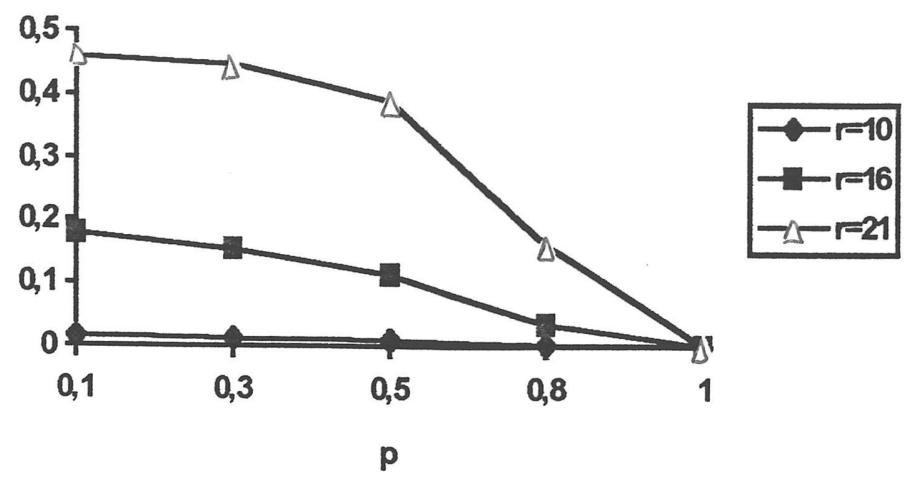

Figura 5.36: divergência de Kullback-Leibler considerando $N=30$

$\mathrm{Na}$ figura (5.36), para $\mathrm{p}=0.10$ e $\mathrm{r}=16, \mathrm{KL}\left(\pi_{\mathrm{o}}, \pi\right)=0.18$, estamos comparando uma $B(0.5)$ com uma $B(0.77)$; e para $\mathrm{p}=0.10$ e $\mathrm{r}=21, \mathrm{KL}\left(\pi_{\mathrm{o}}, \pi\right)=$ 0.46 , estamos comparando uma $B(0.5)$ com uma $B(0.89)$. Portanto, podemos dizer que as distribuições $\pi_{0} \mathrm{e} \pi$ não são similares.

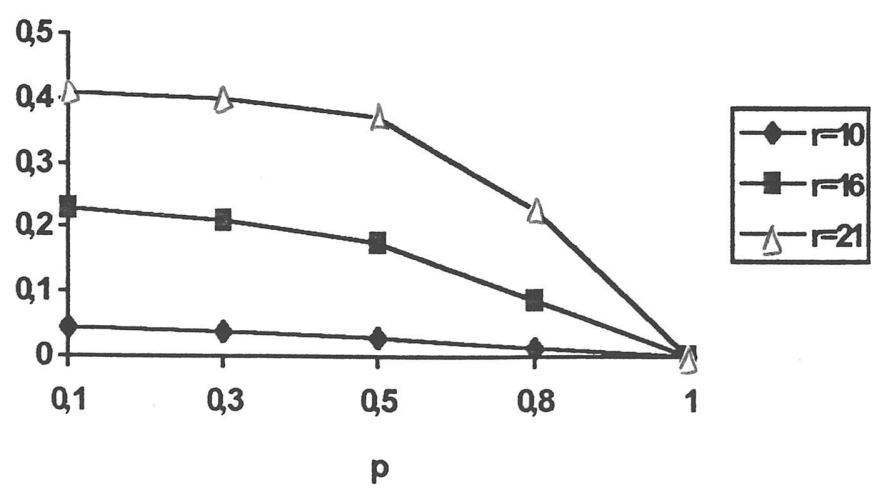

Figura 5.37: Variação Total considerando $N=30$

Na figura (5.37), para $\mathrm{r}=16 \mathrm{e} \mathrm{p}=0.1, \operatorname{VT}\left(\pi_{0}, \pi\right)=0.23$, temos então uma diferença moderada; e para $\mathrm{r}=21$ e $\mathrm{p}=0.1,0.3$ e $0.5,0.37<\mathrm{VT}\left(\pi_{0}, \pi\right)<0.41$. Para esses valores, temos uma grande diferença entre as posteriores. 


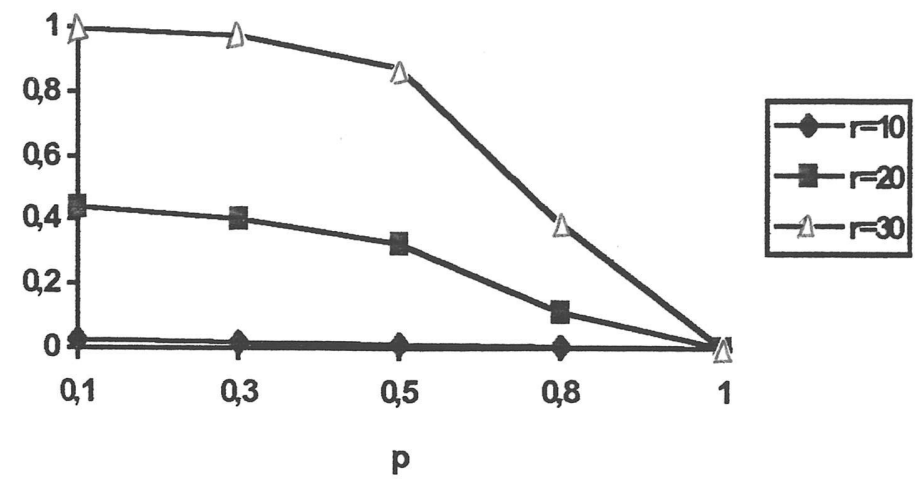

Figura 5.38: divergência de Kullback-Leibler considerando $N=40$

$\mathrm{Na}$ figura (5.38), para $\mathrm{p}=0.10$ e $\mathrm{r}=20, \operatorname{KL}\left(\pi_{0}, \pi\right)=0.44$, estamos comparando uma $B(0.5)$ com uma $B(0.88)$; e para $\mathrm{p}=0.10$ e $\mathrm{r}=30, \mathrm{KL}\left(\pi_{\mathrm{o}}, \pi\right)=$ 0.995 , estamos comparando uma $B(0.5)$ com uma $B(0.96)$. Portanto, podemos dizer que as distribuições $\pi_{0}$ e $\pi$ são diferentes.

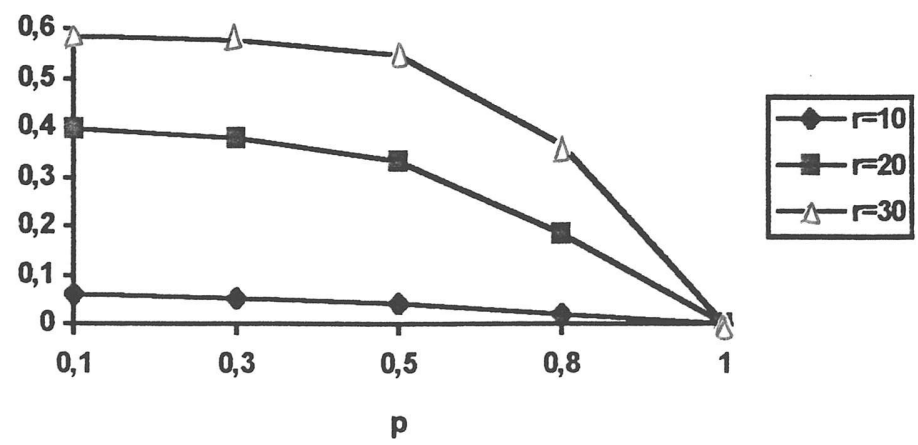

Figura 5.39: Variação Total considerando $N=40$

Na figura (5.39), para $r=20$ e $p=0.1, \operatorname{VT}\left(\pi_{0}, \pi\right)=0.395$; e para $r=30$ e todos os valores de $\mathrm{p}, 0.37<\mathrm{VT}\left(\pi_{0}, \pi\right)<0.59$. Para todos esses valores, temos uma grande diferença entre as posteriores. 


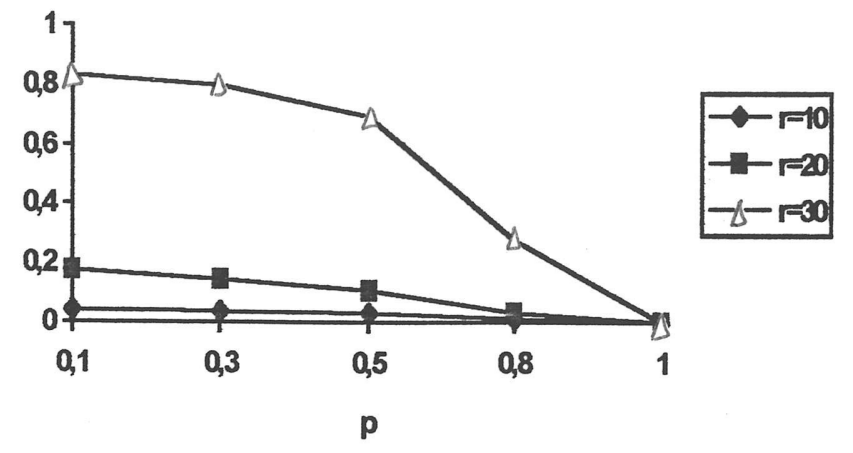

Figura 5.40: divergência de Kullback-Leibler considerando $N=50$

No gráfico da figura (5.40), para $\mathrm{p}=0.10$ e $\mathrm{r}=20, \mathrm{KL}\left(\pi_{0}, \pi\right)=0.17$, estamos comparando uma $B(0.5)$ com uma $B(0.77)$; e para $\mathrm{p}=0.10$ e $\mathrm{r}=30, \mathrm{KL}\left(\pi_{\mathrm{o}}, \pi\right)=$ 0.83 , estamos comparando uma $B(0.5)$ com uma $B(0.95)$. Portanto, podemos dizer que as distribuições $\pi_{0} \mathrm{e} \pi$ são diferentes.

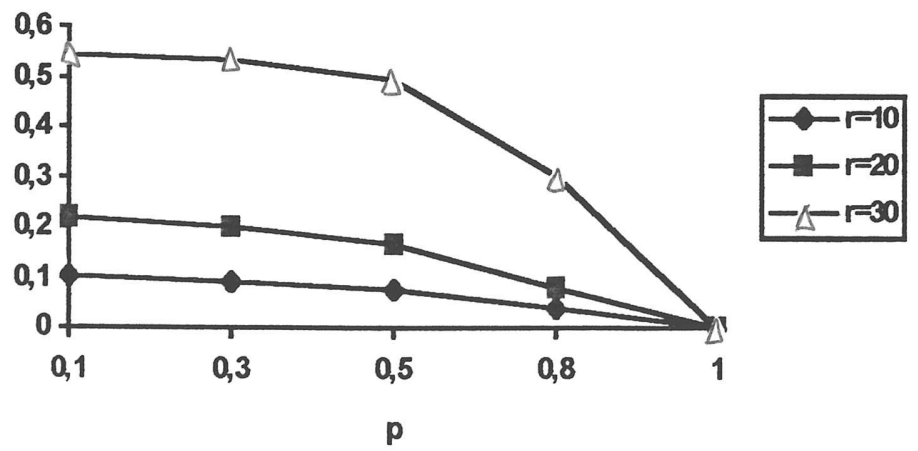

Figura 5.41: Variação Total considerando $N=50$

No gráfico da figura (5.41), para $\mathrm{r}=20 \mathrm{e} \mathrm{p}=0.1, \mathrm{VT}\left(\pi_{0}, \pi\right)=0.22$, temos uma diferença moderada; e para $\mathrm{r}=30$ e todos os valores de $\mathrm{p}, 0.30<\mathrm{VT}\left(\pi_{0}, \pi\right)<0.54$. Para esses valores, temos uma grande diferença entre as posteriores. 


\section{CAPÍTULO 6}

\section{CONCLUSÕES FINAIS E PERSPECTIVAS FUTURAS}

O modelo de Captura-Recaptura de Nayak (1988) pode ser obtido utilizandose processos de Poisson, sem a necessidade de observarmos os tempos de falhas,pois somente o número total de falhas $(\mathrm{R}+\mathrm{M})$ e o número de erros $(\mathrm{R})$ que as causam são relevantes.

Os contadores $\mathrm{M}_{\mathrm{i}}$ introduzidos no modelo de Captura-Recaptura dão uma informação adicional na estimativa dos parâmetros, pois se retirarmos os contadores, teremos o modelo de Jelinski e Moranda. Além de dar uma informação a mais, o contador é de extrema importância na análise Bayesiana quando utilizamos densidades a priori não informativas. Com os contadores no modelo, não teremos problemas de inexistência da densidade a posteriori.

Quando utilizamos o modelo de Goel e Okumoto, onde $\mathrm{p}<1$, os tempos de falhas são relevantes, ou seja, nos dão alguma informação sobre o parâmetro do modelo. Aqui está a razão de os tempos de falhas no modelo de captura-recaptura de Nayak não serem necessários, pois o modelo de Nayak assume $\mathrm{p}=1$.

Os algoritmos de Gibbs Sampling e Metropolis-Hastings são de grande utilidade quando queremos calcular densidades a posteriori marginais. Essa técnica 
de simulação resolve o grande problema da inferência Bayesiana que é a resolução de integrais, que, na maioria das vezes, não apresentam soluções analíticas explícitas.

Levando em conta os critérios sugeridos por McCulloch (1989) para a divergência de Kullback-Leibler e Peng e Dey (1995) para a Variação Total, concluímos, para o conjunto de dados gerados, que, à medida que $\mathrm{r}$ aumenta, ou seja, quanto maior o número de erros encontrados no software, maior será a diferença entre as densidades a posteriori marginais de $\mathrm{N}$.

Para $\mathrm{r}$ próximo de $\mathrm{N}$, temos que, para o conjunto de dados gerados, a distribuição a posteriori $\pi_{0}$ não é robusta, ou seja, precisamos nos preocupar com a correção imperfeita $(\mathrm{p}<1)$. Portanto, é de fundamental importância que 0 pesquisador tenha a certeza de estar utilizando o valor correto de $\mathrm{p}$; caso contrário, sua distribuição a posteriori marginal de $\mathrm{N}$ será totalmente diferente da verdadeira.

Em relação às perspectivas futuras, destacamos a necessidade de

1. considerar outros modelos de confiabilidade e também outras densidades a priori para os parâmetros;

2. propor algum método para a comparação desses diferentes modelos de confiabilidade de software;

3. utilização de outras medidas de divergência, por exemplo, a divergência quiquadrado e a distância de Hellinger. 


\section{APÊNDICE 1}

\section{O Método de Newton}

Suponhamos que desejamos encontrar a raiz $\hat{\theta}$ da equação $g(\theta)=0$. Seja $\theta_{0}$ um valor fixo para $\theta$. Expandindo $g(\theta)$, através de uma série de Taylor no ponto $\theta_{0}$, temos

$$
\mathrm{g}(\theta)=\mathrm{g}\left(\theta_{0}\right)+\left(\theta-\theta_{0}\right) \mathrm{g}^{\prime}\left(\theta_{0}\right)+\frac{\left(\theta-\theta_{0}\right)^{2} \mathrm{~g}^{\prime \prime}\left(\theta_{0}\right)}{2 !}+\ldots
$$

Para $\left|\theta-\theta_{0}\right|$ pequeno, os termos n-ésimos na expressão acima são pequenos e, eliminando-se estes termos, temos

$$
\mathrm{g}(\theta) \approx \mathrm{g}\left(\theta_{0}\right)+\left(\theta-\theta_{0}\right) \mathrm{g}^{\prime}\left(\theta_{0}\right)
$$

Portanto, aproximamos $g(\theta)$ por uma função linear de $\theta$. Como $g(\theta)=0$, obtemos

$$
\mathrm{g}\left(\theta_{0}\right)+\left(\theta-\theta_{0}\right) \mathrm{g}^{\prime}\left(\theta_{0}\right) \approx 0
$$

ou,

$$
\theta_{1} \approx \theta_{0}-\frac{\mathrm{g}\left(\theta_{0}\right)}{\mathrm{g}^{\prime}\left(\theta_{0}\right)}
$$

No método de Newton, tomamos $\theta_{0}$ como sendo uma condição inicial para $\theta$ e o valor de $\theta$ na etapa seguinte, $\theta_{1}$, é dado pela expressão acima. 
Repetindo os cálculos, tomando $\theta_{1}$ como condição inicial, temos

$$
\theta_{2} \approx \theta_{1}-\frac{\mathrm{g}\left(\theta_{1}\right)}{\mathrm{g}^{\prime}\left(\theta_{1}\right)}
$$

Continuamos este procedimento até $\theta_{\mathrm{i}+1} \approx \theta_{\mathrm{i}}$, neste caso, $\mathrm{g}\left(\theta_{\mathrm{i}}\right)=0$ e a raiz $\hat{\theta}$ é dada por $\theta_{\mathrm{i}}$.

No caso de dois parâmetros, nós precisamos resolver um par de equações simultâneas,

$$
\mathrm{g}(\alpha, \beta)=0 ; \quad \mathrm{h}(\alpha, \beta)=0 .
$$

Começando com um valor inicial $\left(\alpha_{0}, \beta_{0}\right)$, nós repetimos a expressão dada abaixo até obtermos a convergência, ou seja, até $\left(\alpha_{i+1}, \beta_{i+1}\right) \approx\left(\alpha_{i}, \beta_{i}\right)$.

$$
\left[\begin{array}{l}
\hat{\alpha} \\
\hat{\beta}
\end{array}\right] \approx\left[\begin{array}{l}
\alpha_{0} \\
\beta_{0}
\end{array}\right]-\left[\begin{array}{ll}
\frac{\partial \mathrm{g}}{\partial \alpha} & \frac{\partial \mathrm{g}}{\partial \beta} \\
\frac{\partial \mathrm{h}}{\partial \alpha} & \frac{\partial \mathrm{h}}{\partial \beta}
\end{array}\right]^{-1}\left[\begin{array}{l}
\mathrm{g} \\
\mathrm{h}
\end{array}\right] .
$$

Esta generalização do método de Newton é chamada de método de NewtonRaphson (ver Kalbfleisch, 1985). 


\section{APÊNDICE 2}

\section{Obtenção da Priori de N}

A seguir, mostramos como foi obtida a priori para $\mathrm{N}$ em (3.27). A distribuição Binomial Negativa com parâmetros $\left(a_{2}, p\right)$ é dada por

$$
P(Y=y)=\left(\begin{array}{c}
y-1 \\
a_{2}-1
\end{array}\right) p^{a_{2}}(1-p)^{y-a_{2}}, y=a_{2}, a_{2}+1, a_{2}+2, \ldots
$$

Se fizermos $y=\left(N+a_{2}\right)$ e $p=\left[b_{2} /\left(b_{2}+1\right)\right]$, teremos

$$
P(Y=N)=\left(\begin{array}{c}
N+a_{2}-1 \\
a_{2}-1
\end{array}\right)\left(\frac{b_{2}}{b_{2}+1}\right)^{a_{2}}\left(1-\frac{b_{2}}{b_{2}+1}\right)^{N}, N=0,1,2, \ldots
$$

Densidade a Priori de N (utilizando o modelo hierárquico):

$$
\begin{aligned}
& p(N)=\int_{0}^{\infty} \frac{e^{-\mu} \mu^{N}}{N !} \frac{\mu^{a_{2}-1} e^{-b_{2} \mu} b_{2}^{a_{2}}}{\Gamma\left(a_{2}\right)} d \mu=\frac{b_{2}{ }^{a_{2}}}{N ! \Gamma\left(a_{2}\right)} \int_{0}^{\infty} \mu^{N+a_{2}-1} e^{-\left(b_{2}+1\right) \mu} d \mu \\
& =\frac{b_{2}^{a_{2}} \Gamma\left(N+a_{2}\right)}{\Gamma\left(a_{2}\right) N !\left(b_{2}+1\right)^{N+a_{2}}} \int_{0}^{\infty} \frac{\left(b_{2}+1\right)^{N+a_{2}}}{\Gamma\left(N+a_{2}\right)} \mu^{N+a_{2}-1} e^{-\left(b_{2}+1\right) \mu} d \mu=\frac{b_{2}{ }^{a_{2}} \Gamma\left(N+a_{2}\right)}{\Gamma\left(a_{2}\right) N !\left(b_{2}+1\right)^{N+a_{2}}} \\
& =\left(\begin{array}{c}
N+a_{2}-1 \\
a_{2}-1
\end{array}\right)\left(\frac{b_{2}}{b_{2}+1}\right)^{a_{2}}\left(1-\frac{b_{2}}{b_{2}+1}\right)^{N} \propto \frac{\Gamma\left(N+a_{2}\right)}{N !\left(b_{2}+1\right)^{N+a_{2}}} .
\end{aligned}
$$




\section{APÊNDICE 3}

\section{Alguns Programas Desenvolvidos}

A seguir, mostramos alguns programas desenvolvidos no pacote estatístico Minitab e no Matlab.

1 - Programa desenvolvido no Minitab para obtenção das amostras de $\mathrm{N}^{\prime}$ e $\lambda$ no modelo de Jelinski e Moranda, utilizando o algoritmo de Gibbs Sampling.

set $\mathrm{c} 1$

92132364345505863707177788791929598104105116149156247

249250

end

write 'data 1' c1

let $\mathrm{k} 1=3.5$

let $\mathrm{k} 2=0.02$

read $\mathrm{c} 10 \mathrm{c} 11$

00

end

sum c1 k3

store 'a'

no echo

end

exec 'c:gibbs.pro' 999

desc c 10 c 11

dotplot c10 c11

programa gibbs.pro

let $\mathrm{k} 4=1 /(3294.11+250 * \mathrm{k} 1)$

random $3 \mathrm{c} 2$;

gamma $31.49 \mathrm{k} 4$.

let $\mathrm{k} 2=\mathrm{c} 2(2)$

print $\mathrm{c} 2$ 


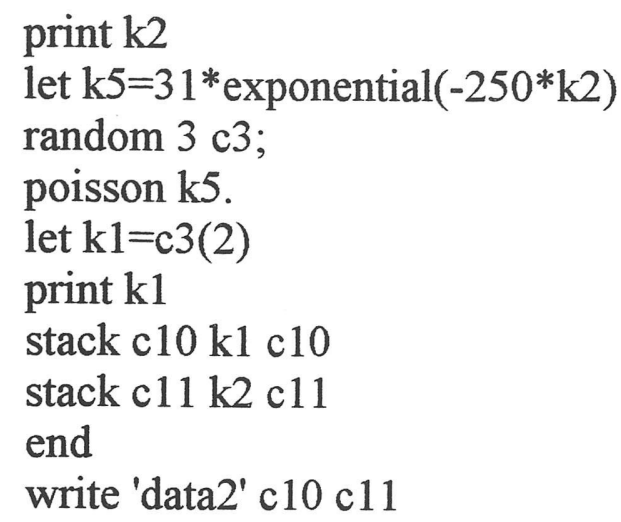

2 - Programa desenvolvido no Matlab para obtenção das amostras de $\mathrm{N}, \lambda$ e $p$ no modelo de Goel e Okumoto com captura-recaptura ( $\mathrm{p}$ desconhecido), utilizando o algoritmo de Gibbs Sampling com Metropolis-Hastings. O critério da convergência e os intervalos de credibilidade também estão neste programa.

clear;

rand('seed', 0 );

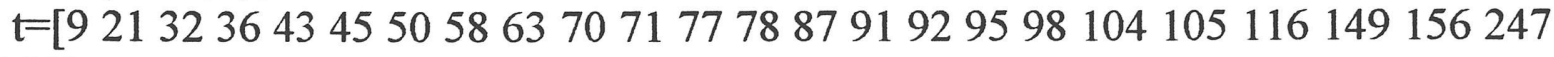
249 250];

$\mathrm{a} 1=2.5$;

$\mathrm{b} 1=2.5$;

$\mathrm{a} 4=5.49$;

b4=802.11;

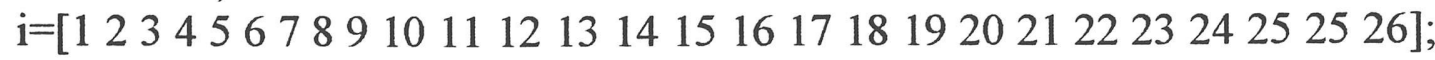

cont $=0$;

cont $2=0$;

$\%$

$\%$ Condições Iniciais

$\%$

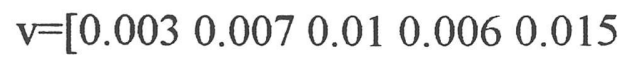

2629333640

$\left.\begin{array}{llllll}0.1 & 0.3 & 0.5 & 0.7 & 0.9\end{array}\right]$;

for $\mathrm{k}=2: 10000$ 
if rem(k-2,2000) $==0 \& \mathrm{k}<2000$

Lambda $(\mathrm{k}-1)=\mathrm{v}(1,1+(\mathrm{k}-2) / 2000)$;

$\mathrm{N}(\mathrm{k}-1)=\mathrm{v}(2,1+(\mathrm{k}-2) / 2000)$;

$\mathrm{p}(\mathrm{k}-1)=\mathrm{v}(3,1+(\mathrm{k}-2) / 2000)$;

end

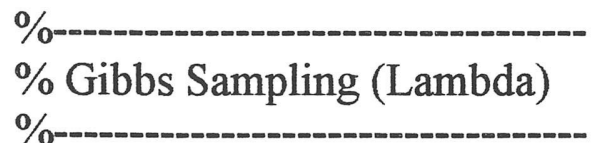

$\mathrm{A}=58+\mathrm{a} 4$;

$\mathrm{B}=1 /(\mathrm{b} 4+(300 * \mathrm{~N}(\mathrm{k}-1))+((1-\mathrm{p}(\mathrm{k}-1)) * 5308))$;

Lambda $(\mathrm{k})=$ gamrnd $(\mathrm{A}, \mathrm{B})$;

$\%$

$\%$ Metropolis-Hastings (N)

$\%$

$y=$ poissrnd $(31)$;

$\mathrm{psi}=\exp (\operatorname{sum}(\log (\mathrm{y}-\mathrm{p}(\mathrm{k}-1) *(\mathrm{i}-1)))-(300 * \operatorname{Lambda}(\mathrm{k}) * \mathrm{y})-\operatorname{sum}(\log (\mathrm{N}(\mathrm{k}-1)-\mathrm{p}(\mathrm{k}-1) *(\mathrm{i}-$

1)) $)+(300 * \operatorname{Lambda}(\mathrm{k}) * \mathrm{~N}(\mathrm{k}-1)))$;

$\mathrm{m}=\min (1, \mathrm{psi})$;

rand('uniform');

um=rand;

if $\mathrm{um}<=\mathrm{m}$

$\mathrm{N}(\mathrm{k})=\mathrm{y}$;

cont $=$ cont +1 ;

else

$\mathrm{N}(\mathrm{k})=\mathrm{N}(\mathrm{k}-1)$;

end

$\%$

$\%$ Metropolis-Hastings ( $\mathrm{p}$ )

$\%$

$\mathrm{z}=$ betarnd $(\mathrm{a} 1, \mathrm{~b} 1)$;

psi2 $=\exp \left(\operatorname{sum}\left(\log \left(\mathrm{N}(\mathrm{k})-\mathrm{z}^{*}(\mathrm{i}-1)\right)\right)-\left(\operatorname{Lambda}(\mathrm{k}) *(1-\mathrm{z})^{*} 5308\right)-\operatorname{sum}(\log (\mathrm{N}(\mathrm{k})-\mathrm{p}(\mathrm{k}-\right.$

$\left.\left.\left.1)^{*}(\mathrm{i}-1)\right)\right)+(\operatorname{Lambda}(\mathrm{k}) *(1-\mathrm{p}(\mathrm{k}-1)) * 5308)\right)$;

$\mathrm{m} 2=\min (1, \mathrm{psi} 2)$;

rand('uniform');

um2=rand; 


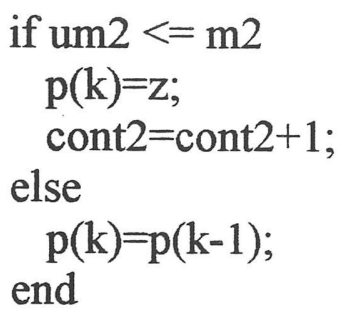

end;

$\%$

\% seleção dos dados gerados

$\%$

$1=1$;

for $\mathrm{j}=1001: 2000: 10000$;

for $\mathrm{r}=\mathrm{j}: \mathrm{j}+999$;

if $\operatorname{rem}(\mathrm{r}-\mathrm{j}+1,5)=0$

$\operatorname{Lambdas}(1)=\operatorname{Lambda}(\mathrm{r})$;

$\mathrm{Ns}(1)=\mathrm{N}(\mathrm{r})$;

$\operatorname{ps}(1)=\mathrm{p}(\mathrm{r})$;

$1=1+1$;

end

end

end

$\%$

$\%$ critério de convergência de Gelman e Rubin

$\%$

fi $1 \mathrm{x}=\mathrm{Ns}$;

fi $2 \mathrm{x}=$ Lambdas;

fi $3 x=p s$;

$\mathrm{nc}=5$;

$\mathrm{n} 1=1$;

$\mathrm{n} 2=$ length $(\mathrm{fi} 1 \mathrm{x}) / \mathrm{nc}$;

$\mathrm{d}=\mathrm{n} 2-\mathrm{n} 1+1$

Mefi1 $=[\operatorname{mean}($ fi $1 x(n 1: n 2))$, mean $($ fi $1 x(n 1+d: n 2+d))$, mean $($ fi $1 x(n 1+2 * d: n 2+2 * d))$, me $\operatorname{an}($ fi $1 x(n 1+3 * d: n 2+3 * d))$, mean(fi $1 x(n 1+4 * d: n 2+4 * d))]$;

QMfi1=Mefi1.*Mefi1;

Vafi $1=[\operatorname{cov}($ fi $1 x(n 1: n 2)), \operatorname{cov}($ fi $1 x(n 1+d: n 2+d)), \operatorname{cov}($ fi $1 x(n 1+2 * d: n 2+2 * d))$, $\operatorname{cov}(\mathrm{fi} 1 \mathrm{x}(\mathrm{n} 1+3 * \mathrm{~d}: \mathrm{n} 2+3 * \mathrm{~d})), \operatorname{cov}(\mathrm{fi} 1 \mathrm{x}(\mathrm{n} 1+4 * \mathrm{~d}: \mathrm{n} 2+4 * \mathrm{~d}))]$ 
MeMfil=sum(Mefi1)/nc;

Wfi1=sum(Vafi1)/nc;

VarVfi1 $=($ Vafi $1 *$ Vafi $1 '-$ nc*Wfi $1 \wedge 2) /($ nc-1);

Ufi1 $=d^{*}\left(\right.$ Mefi1 ${ }^{*}$ Mefi1 $1 '$-nc*MeMfi1^2)/(nc-1);

Sigfi $1=((\mathrm{d}-1) / \mathrm{d}) * \mathrm{Wfi} 1+(1 / \mathrm{d}) *$ Ufi1;

VHfi $1=$ Sigfi $1+$ Ufi $1 /\left(n c^{*} d\right)$;

Mcovfi1= $\operatorname{cov}($ Vafi1,Mefi1);

Qcovfi1 $=\operatorname{cov}($ Vafi1, QMfi1);

COVfil $=\mathrm{Q}$ covfi $1(1,2)-2 * \operatorname{MeMfi} 1 * \operatorname{Mcovfi} 1(1,2)$;

$\operatorname{VarVHfi} 1=((\mathrm{d}-1) / \mathrm{d})^{\wedge} 2 *(1 / \mathrm{nc}) * \operatorname{VarVfi} 1+\left(((\mathrm{nc}+1) /(\mathrm{nc} * \mathrm{~d}))^{\wedge} 2\right)^{*}(2 /(\mathrm{nc}-$

1))*Ufi $1^{\wedge} 2+2 * \operatorname{COVfi1} 1 *(\mathrm{nc}+1) *(\mathrm{~d}-1) /\left(\mathrm{d}^{*} \mathrm{nc}^{\wedge} 2\right)$;

dffi $1=2 *\left(V_{H}{ }^{\prime} 1^{\wedge} 2\right) /$ VarVHfi 1 ;

Rfi1=sqrt((VHfi1/Wfi1)*dffi1/(dffi1-2));

Mefi2 $=[\operatorname{mean}($ fi $2 x(n 1: n 2))$, mean(fi2 $x(n 1+d: n 2+d))$, mean(fi2 $x(n 1+2 * d: n 2+2 * d))$, me an(fi2x(n1+3*d:n2+3*d)), mean(fi2x(n1+4*d:n2+4*d))];

QMfi2=Mefi2.*Mefi2;

Vafi2 $=[\operatorname{cov}($ fi2 $x(n 1: n 2)), \operatorname{cov}($ fi $2 x(n 1+d: n 2+d)), \operatorname{cov}(f i 2 x(n 1+2 * d: n 2+2 * d))$, $\operatorname{cov}($ fi $2 x(n 1+3 * d: n 2+3 * d)), \operatorname{cov}($ fi $2 x(n 1+4 * d: n 2+4 * d))]$;

MeMfi2=sum(Mefi2)/nc;

Wfi2=sum(Vafi2)/nc;

VarVfi2 $=($ Vafi2 $*$ Vafi2'-nc*Wfi2^2)/(nc-1);

Ufi2 $=d^{*}($ Mefi2 $*$ Mefi2'-nc*MeMfi2^2)/(nc-1);

Sigfi $2=((\mathrm{d}-1) / \mathrm{d}) * \mathrm{Wfi} 2+(1 / \mathrm{d}) * \mathrm{Ufi} 2$;

VHfi2=Sigfi2+Ufi $/\left(\mathrm{nc}^{*} \mathrm{~d}\right)$;

Mcovfi2=cov(Vafi2,Mefi2);

Qcovfi2 $=\operatorname{cov}($ Vafi2, QMfi2);

COVfi2 $=$ Qcovfi2 $(1,2)-2 *$ MeMfi2 $*$ Mcovfi2 $(1,2)$;

$\operatorname{VarVHfi} 2=((\mathrm{d}-1) / \mathrm{d}) \wedge 2 *(1 / \mathrm{nc}) * \operatorname{VarVfi} 2+\left(((\mathrm{nc}+1) /(\mathrm{nc} * \mathrm{~d}))^{\wedge} 2\right)^{*}(2 /(\mathrm{nc}-$

1))*Ufi2 ${ }^{\wedge} 2+2 * \operatorname{COVfi} 2 *(\mathrm{nc}+1) *(\mathrm{~d}-1) /\left(\mathrm{d}^{*} \mathrm{nc}^{\wedge} 2\right)$;

dffi2 $=2 *($ VHfi $2 \wedge 2) /$ VarVHfi2;

Rfi2=sqrt((VHfi2/Wfi2)*dffi2/(dffi2-2));

Mefi3 $=[\operatorname{mean}(f i 3 x(n 1: n 2)), \operatorname{mean}(f i 3 x(n 1+d: n 2+d))$, mean $(f i 3 x(n 1+2 * d: n 2+2 * d))$, me an(fi3x(n1+3*d:n2+3*d)), mean(fi3x(n1+4*d:n2+4*d))];

QMfi3=Mefi3.*Mefi3;

Vafi3 $=[\operatorname{cov}($ fi3 $3(n 1: n 2)), \operatorname{cov}(f i 3 x(n 1+d: n 2+d)), \operatorname{cov}(f i 3 x(n 1+2 * d: n 2+2 * d))$, $\operatorname{cov}(f i 3 x(n 1+3 * d: n 2+3 * d)), \operatorname{cov}(f i 3 x(n 1+4 * d: n 2+4 * d))]$;

MeMfi3=sum(Mefi3)/nc;

Wfi3 =sum $($ Vafi3 $) /$ nc;

VarVfi3 $=\left(\right.$ Vafi3 ${ }^{*}$ Vafi3'-nc*Wfi3 $\left.{ }^{\wedge} 2\right) /($ nc-1)

Ufi3 $=d^{*}\left(\right.$ Mefi3 ${ }^{*}$ Mefi3'-nc* MeMfi3^2)/(nc-1); 
Sigfi3 $=((d-1) / d) * W f i 3+(1 / d) * U f i 3$;

VHfi3=Sigfi3+Ufi3/(nc*d);

Mcovfi3= $\operatorname{cov}($ Vafi3,Mefi3);

Qcovfi3 $=\operatorname{cov}($ Vafi3, QMfi3);

COVfi3 $=$ Qcovfi3 $(1,2)-2 *$ MeMfi3 $*$ Mcovfi3 $(1,2)$;

$\operatorname{VarVHfi} 3=((\mathrm{d}-1) / \mathrm{d}) \wedge 2 *(1 / \mathrm{nc}) * \operatorname{VarVfi} 3+\left(((\mathrm{nc}+1) /(\mathrm{nc} * \mathrm{~d}))^{\wedge} 2\right)^{*}(2 /(\mathrm{nc}-$

1))*Ufi $3 \wedge 2+2 * \operatorname{COVfi} 3 *(\mathrm{nc}+1) *(\mathrm{~d}-1) /\left(\mathrm{d}^{*} \mathrm{nc}^{\wedge} 2\right)$;

dffi3 $=2 *\left(\right.$ VHfi3 $\left.{ }^{\wedge} 2\right) /$ VarVHfi3;

Rfi3 $=$ sqrt ((VHfi3/Wfi3)*dffi3/(dffi3-2));

$\operatorname{disp}([R f i 1$, Rfi2, Rfi3])

$\%$

$\%$ intervalos de credibilidade ( $95 \%)$

$\%$

Nord $=$ sort(Ns);

Nord $1=$ sort(Lambdas);

Nord2 $=\operatorname{sort}(\mathrm{ps})$;

$\mathbb{I N s}=[\operatorname{Nord}(0.025 *$ length(Ns) $)$ Nord $(0.975 *$ length(Ns) $)]$

ILambdas $=[\operatorname{Nord} 1(0.025 *$ length $($ Lambdas $))$ Nord $1(0.975 *$ length $($ Lambdas $))]$ $\mathrm{Ips}=[\operatorname{Nord} 2(0.025 *$ length $(\mathrm{ps}))$ Nord2 $(0.975 *$ length $(\mathrm{ps}))]$ 


\section{REFERÊNCIAS BIBLIOGRÁFICAS}

ACHCAR, J. A. (1995). Use of Approximate Bayesian Inference for Software Reliability. Notas do ICMSC, Série Estatística, 18.

ACHCAR, J. A. (1996). Bayesian Inference for Software Reliability Models Considering Interfailure Time Data. Notas do ICMSC, Série Estatística, 30.

BORGES, W. S.; COlOSiMO, E. A.; FREITAS, M. A. (1996). Métodos Estatísticos e Melhoria da Qualidade: Construindo Confiabilidade em Produtos. $12^{\circ}$ Simpósio Nacional de Probabilidade e Estatística.

BICKEL, P. J.; DOKSUM, K. A. (1977). Mathematical Statistics. Basic Ideas and Selected Topics. San Francisco, Holden Day.

BOX, G. E. P.; TIAO, G. C. (1973). Bayesian Inference in Statistical Analysis. New York, Addison-Wesley.

CASELLA, G.; GEORGE, E. I. (1992). Explaining the Gibbs Sampler. The American Statistical Association, 46, 3, 167-174.

CLARKE, A. B.; DISNEY, R. L. (1970). Probability and Random Processes for Engineers and Scientists. John Wiley \& Sons. 
CSISZÁR, I. (1967). Information-Type Measures of Difference of Probability Distributions and Indirect Observations. Studia Sci. Math. Hungar, 2, 299-318.

CHIB, S.; GREENBERG, E. (1995). Understanding the Metropolis-Hastings Algorithm. The American Statistician, 49, 4, 327-335.

DEY, D. K.; BIRMIWAL, L. R. (1994). Robust Bayesian Analysis Using Entropy and Divergence Measures. Statist. Probab. Lett., 20, 287-294.

GAMERMAN, D. (1996). Simulação Estocástica via Cadeias de Markov. $12^{\circ}$ Simpósio Nacional de Probabilidade e Estatística.

GELMAN, A.; RUBIN, D. B. (1992). Inference from Iterative Simulation Using Multiple Sequences. Statistical Science, 7, 4, 457-472.

GOEL, A. L. (1985). Software Reliability Models: Assumptions, Limitations, and Applicability. IEEE Trans. on Software Engineering, 11, 12, 1411-1423.

GOEL, A. L.; OKUMOTO, K. (1978). An Analysis of Recurrent Software Failures on a Real Time Control System. ACM Annu. Tech. Conf., Washington D. C., 496-500.

HASTINGS, W. K. (1970). Monte Carlo Sampling Methods Using Markov Chains and their Applications. Biometrika, 57, 97-109. 
JELINSKI, Z॰; MORANDA, P. B. (1972). Software Reliability Research. Statistical Computer Performance Evaluation, ed. W. Freiberger, New York and London, Academic Press, 465-484.

JOHNSON, W.; GEISSER, S. (1983). A Predictive View of the Detection and Characterization of Influential Observations in Regression Analysis. Journal of the American Statistical Association, 78, 137-144.

KALBFLEISCH, J. D.; PRENTICE, R. L. (1980). The Statistical Analysis of Failure Time Data. New York, John Wiley \& Sons.

KALBFLEISCH, J. G. (1985). Probability and Statistical Inference. Second Edition, New York, Springer-Verlag.

LANGBERG, N.; SINGPURWALLA, N. D. (1985). A Unification of Some Software Reliability Models. Siam J. Sci. Stat. Comput., 6, 3, 781-790.

LAWLESS, J. F. (1982). Statistical Models and Methods for Lifetime Data. New York, John Wiley \& Sons.

McCULLOCH, R. E. (1989). Local Model Influence. Journal of the American Statistical Association, 84, 406, 473-478.

MOOD, A. M.; GRAYBIL, F. A.; BOES, D. C. (1974). Introduction to the Theory of Statistics. Third Edition, New York, McGraw-Hill. 
MORANDA, P. B. (1975). Prediction of Software Reliability and its Applications. Proceedings of the Annual Reliability and Maintainability Symposium, 327332, Washington D. C..

MUSA, J. D.; IANNINO, A.; OKUMOTO, K. (1987). Software Reliability: Measurement, Prediction, Application. McGraw-Hill.

NATARAJAN, R.; MCCULLOCH, C. E. (1995). A Note of the Existence of the Posteriori Distribution for a Class of Mixed Models for Binomial Responses. Biometrika, 82, 3, 639-643.

NAYAK, T. K. (1988). Estimating Population Size by Recapture Sampling, Biometrika, 75, 1, 113-120.

PENG, F.; DEY, D. K. (1995). Bayesian Analysis of Outlier Problems Using Divergence Measures. The Canadian Journal of Statistics, 23, 2, 199-213.

RODRIGUES, J. (1995). Inference for the Software Reliability Using Asymmetric Loss Functions: A Hierarchial Bayes Approach. Série Estatística, ICMSC, 21.

RODRIGUES, J.; LEITE, J. G. (1996). Inference for the Software Reliability Using Imperfect Recapture Debugging Model. Notas do ICMSC, Série Estatística, 28.

SCHICK, G. J.; WOLVERTON, R. W. (1978). Assessment of Software Reliability. Proc. Oper. Res., 395-422. Wirzberg-Wien: Physica-Verlag. 
SEBER, G. A. F. (1982). The Estimation of Animal Abundance. Second Edition, Charles Griffin \& Company Ltd., London.

SINGPURWALLA, N. D.; WILSON, S. P. (1994). Software Reliability Modeling. International Statistical Review, 62, 3, 289-317.

TIERNEY, L.; KADANE, J. B. (1986). Accurate Aproximations for Posteriori Moments and Marginal Densities. Journal of the American Statistical Associations, 81, 393, 82-86. 
